\title{
UNRAVELING PSYCHIATRIC SUB-PHENOTYPES: \\ THE PRICE OF THE REDUCTION \\ OF MYELIN BASIC PROTEIN
}

\author{
Dissertation \\ for the award of the degree \\ "Doctor of Philosophy" (Ph.D.) \\ Division of Mathematics and Natural Sciences \\ of the Georg-August-Universität Göttingen
}

submitted by Giulia Poggi

from Novara - ITALIA

Göttingen 2015 



\section{Members of the Thesis Committee}

Prof. Hannelore Ehrenreich (Supervisor, first reviewer)

Clinical Neuroscience

Max Planck Institute of Experimental Medicine

Hermann-Rein straße 3

37075 Göttingen

Germany

Prof. Klaus-Armin Nave (second reviewer)

Department of Neurogenetics

Max Planck Institute of Experimental Medicine

Hermann-Rein straße 3

37075 Göttingen

Germany

Prof. Mikael Simons

Cellular Neuroscience

Max Planck Institute of Experimental Medicine

Hermann-Rein straße 3

37075 Göttingen

Germany

Date of the thesis submission: 31.10 .2015

Date of the oral examination: 08.01.2016 

I hereby declare that the thesis "UNRAVELING PSYCHIATRIC SUBPHENOTYPES:THE PRICE OF THE REDUCTION OF MYELIN BASIC PROTEIN" has been written independently and with no other sources and aids than quoted.

Göttingen, 31.10.2015

Giulia Poggi 



\section{Acknowledgement}

Someone said that the most complicated part of a PhD thesis is the discussion, where one really needs to show the scientific competence in interpreting data...

Someone else told me: "The introduction... oh God, terrible! After that everything runs smoothly!"

...I am telling you know... going through memories of "how I got here"...

This is probably the most challenging part of this $\mathrm{PhD}$ thesis...

It was a long way and it definitely would not have been the way it was without the people that "walked" with me...

To these people, I express my infinite gratitude...

...Prof. Hannelore Ehrenreich, for giving me the unique possibility of doing what I love: SCIENCE! These four years have been tough, but they enriched me as scientist and, most importantly, as a person. I will be eternally grateful to you.

...Prof. Nave and Prof. Simons, for your irreplaceable knowledge on myelin, andfor your scientific support and discussion.

...my Examination Board, for being present on this important day and for contributing with extremely valuable knowledge and insights to my defense.

...Prof. Boretius and Dr. Baudewig, for the fruitful collaboration with amazing people and great scientists: the time and the work with you have been among the main highlights of my whole $\mathrm{PhD}$ time!

...Dr. Möbius and Torben, for the infinite knowledge and for the even bigger patience in each moment of panic that I had for my EM samples ;)

...Prof. Fisher, Dr. Sananbenesi, Ms.Susanne Burkhardt, for the enriching collaboration that gave me more insights into the "miRNAs world".

...Dr. Zafeiriou, for the irreplaceable support and the scientific discussion on the "miRNAs machinery".

...Wiebke, for being the master organiser of EVERYTHING in our group, nothing would work without you!

...Karla, for any chat in German, that I did NOT understand, but made me smile and have nice time anyway, for always saving us from the KRAKEN in the kitchen, for 
being one of the major collaborators for my cloning (how many litres of LB did you autoclave???)...

...Martesa, for being like a postdoc to me even before getting your $\mathrm{PhD}$ and being a very special friend. Thank you for any scientific discussion, for any cigarette, for any coffee in the MPI during weekends, for listening always to my "dramas" and for being always present, even if now we are apart. I miss you so much.

...Sergi, for being "my postdoc": a great guide and a unique person! Thank you for "the postdoc sign" that allowed us to leave the table after lunch, for "today is pizza-day", for having ALWAYS the time to help/teach/support me...and thank you for BONFERRONI correction!!

...Bárbara or maybe I should say Olivia! Thank you for being who you are... for sharing good and bad moments, for the chopstick fights in the kitchen, for the Danish cheese, for Columbus, for showing me how cool ACDC are, for laughing non-stop after 15 hours of work in the lab, for the "baby don't grow, no mold", for cycling with a hello-kitty pillow, for giving me awesome dresses that make me look like a woman (it was about time for me!!), for knowing me... and still being my friend ;) ! I will miss you, Olivia...really!

...Marie, for being crazy in love with MBP together with me ;), for "ich zahle acht", for trying to adapt to the Mediterranean style, for the special way of removing surplus ice from the freezer, for saving all of us when we are not able to communicate (your German is quite fluent, you know...;P ), for cycling at the speed of light (and getting home even before I get on my bike), for being the friend you are!

...Gabriela, for a T-rex of $1 \mathrm{~kb}$ that... was a control!! For sleeping on the couch, for the 3 SpeI sites, for the T4 ligase-ninjas, for getting on the elevator while shaking the tail (and seeing unexpected horrified faces!!)... and for many other things I will not mention here, otherwise I will never get a $\mathrm{PhD}$;) !! I enjoyed any single moment of the cloning marathon and I found a very good friend!

...Marina, for how easy is to get along with you at work and in life; for the bushes we had to cross (it was worth doing it!), for the giant-moka\&nocino night, for the lovely time when you came to Göttingen the first, the second and the third time... I am really happy to have met you!!

...Artem, well... for being you and for being with me, whatever happens...

...Ludo, for reading my thesis...more than once, even the RESULTS (awful first version!!), for making coffee, for bringing ice-cream/bounty to poor and desperate $\mathrm{PhD}$ students ;), for priceless hairstyle and clothes, for tuna\&beans, for not killing me when I hid your meat, for your MICROGLIA identity that gives us always priceless moments. 
... Anes, Esther, Lilli, Bekir, Swetlana (TOM TOM of the holy knowledge!), Fernanda, Mati, Kati, Dorota and the whole Clinical Neuroscience: Ekrem, Martin, Daniela, Anja, Christoph, Beata, Viktoria, Imam, Nadine, Debia, Hong, Bilke, Nicole, Jan, Anne ...for being perfect colleagues and great people. Thank you very much for the scientific discussions and also for the time together!

...Mara, Ilaria, Edda, Chiara, Giovanna and Livia...the Italian circle in Göttingen...well, how could I have survived without you? Ladybug and bee, oompa loompa, banda bassotti, piscio fritto, "facciamo il vento", the diet from Monday, il saluto tedesco, Achille that was actually a female rat, le "figure di merda" a propulsione and one million memories more...you are great!! I am so glad to have met you!!

...Carlina, for being still with me after all these years, even if we cannot see each other so often, for being the strong woman you are, a woman who does not give up in front of anything; for being more than a friend, for being my best friend. Ti voglio un bene infinito e te ne vorrò sempre!!

...Ramona, or maybe I should say waccs ;), for any single moment of these 10-year marriage, for "verso l'infinito ed oltre", for year 2006 but also for whatever came afterwards, for being the friend who always backs me up, for messing up with me like there is no tomorrow, for knowing exactly what I want to say, even before I think of it...

...Ester, for being a faithful friend and one of the few survivors from the past, for being supportive and always present, for always making some time for me...

...Patti, Doro and the whole ASC team... for some relaxing sport time out of the lab :)!

...Pusi, for being my friend, in spite of everything... for making me feel that, even if we don't see each other for long time, once we meet again... it always feels like home.

...Ale, for having been a pleasant surprise a few years ago: for every moment we shared, for Hamburg, for dressing up and running around Göttingen at $-10^{\circ} \mathrm{C}$, for having gone from being someone I would hardly talk with to being one of my best friends.

...Tolga, for being Charlie, which includes everything; for our first meeting at the airport... and the second one at your party, for the milka cookies, for pancakes (to be pronounced in the Italian way), for Cinderella, for the boring movies you sometimes propose ;)...

And also for being a great scientist: you have no idea how much I learnt from you in this last year!! 
...Last but not least, my family, the real engine that made me reach this goal; for who is still physically with me and for who is not here anymore: thank you, because I really owe you everything!

Mamma\&Papo...I could not have asked for better parents than you are. Sometimes (I must say, often) we fight, sometimes you probably wonder why you encouraged me to start speaking when I was a child, but I know you are always here to help me and support me. If I made it here, it is because I know that there is a huge amount of love and care I can always count on.

Thank you all, I would not be who I am and I would not have made it here, if I had not met you! 


"Curiouser and curiouser!"

Alice in Wonderland

Lewis Carroll 



\section{TABLE OF CONTENT}

PREFACE

1.SUMMARY

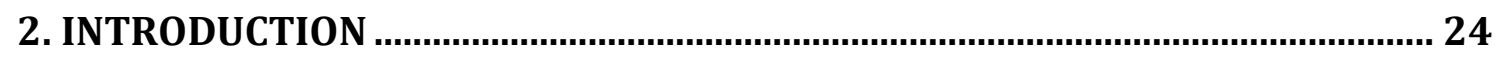

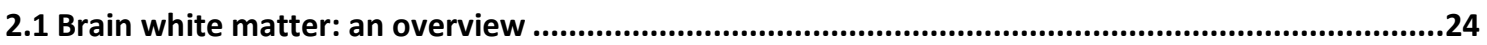

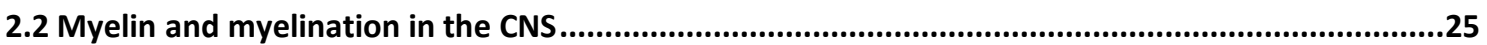

2.2.1.Oligodendrocytes precursor cells and mature oligodendrocytes ........................................25

2.2.2. Myelin biogenesis: processes elongation and axon ensheathment....................................27

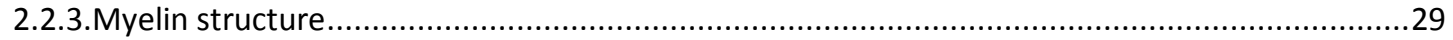

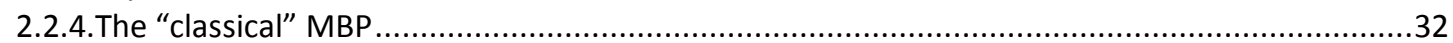

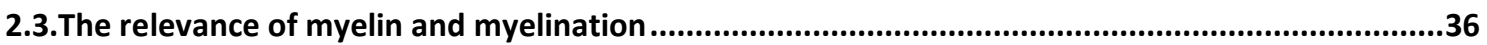

2.4.Myelin abnormalities in schizophrenia: focus on MBP ...........................................................39

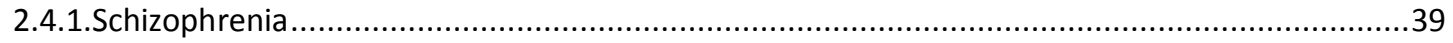

2.4.2. Myelin abnormalities in schizophrenia patients ....................................................... 41

2.5.Shiverer heterozygous: a model to unravel schizophrenia sub-phenotype?..................................45

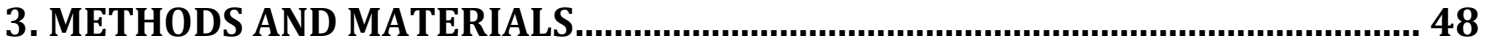

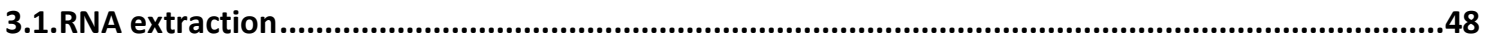

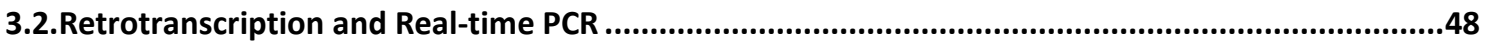

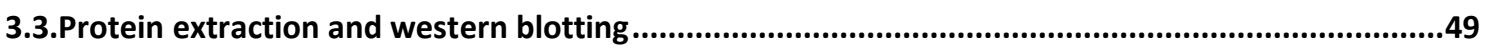

3.4.MRI (in collaboration with Prof. Boretius, who provided the method part)..................................49

3.5.Electron Microscopy (performed in collaboration with Dr. Möbius) ............................................50

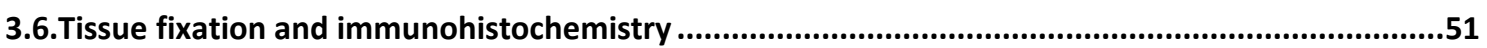

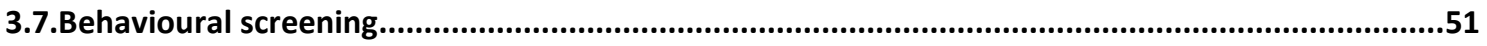

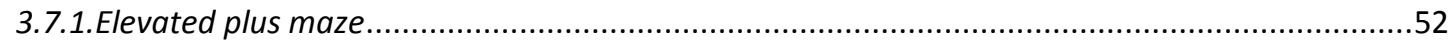

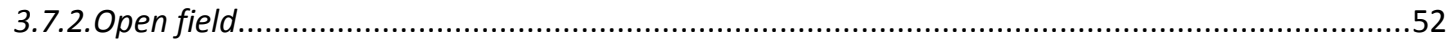

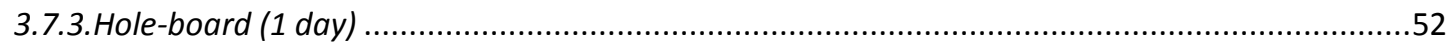

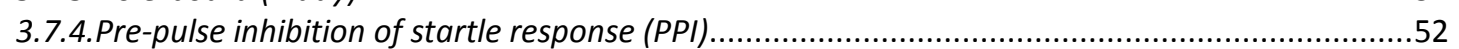

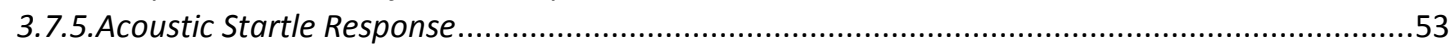

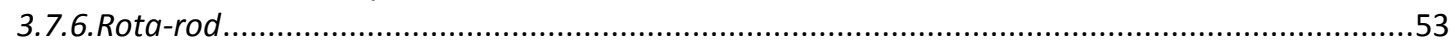

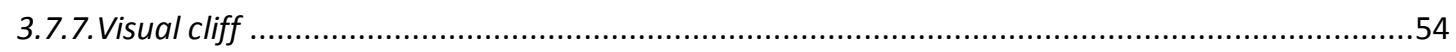

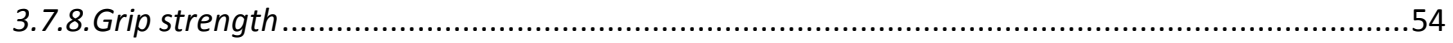

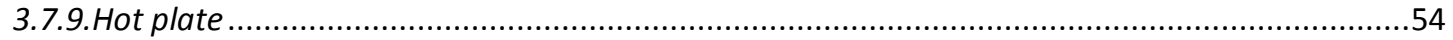

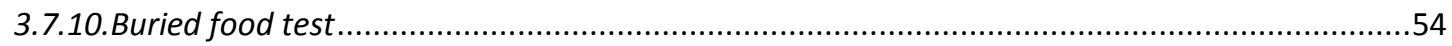

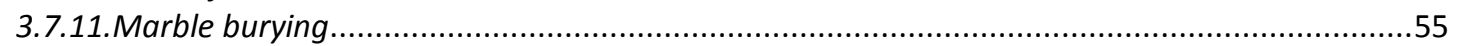

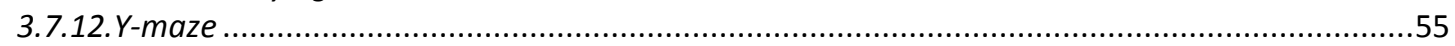

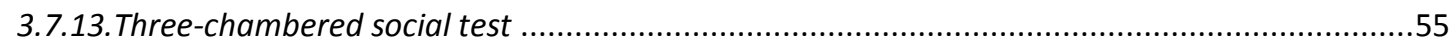

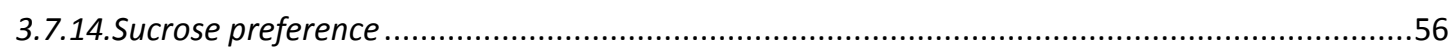

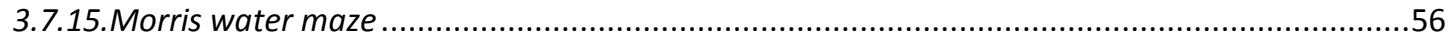




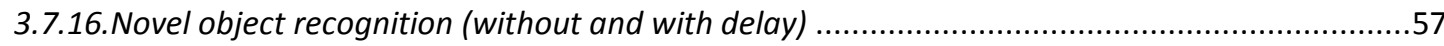

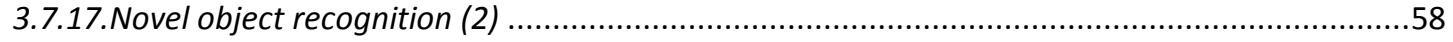

3.7.18. LABORAS - spontaneous home cage behaviour .........................................................58

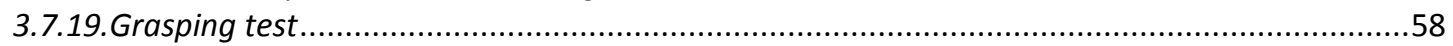

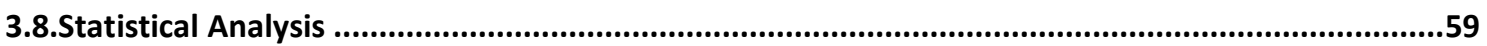

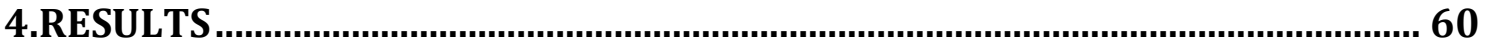

4.1.MBP show an age-dependent and area-specific expression pattern ........................................60

4.2.The lack of one allele coding for MBP does not have drastic effects on the expression of other myelin-related molecules and on oligodendrocyte number ..............................................................64

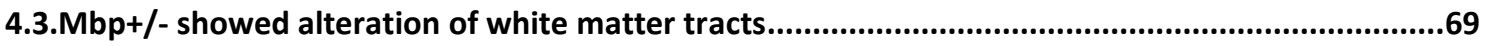

4.4.The rostral corpus callosum in $\mathrm{Mbp}+/$ - showed a trend in reduction of myelin thickness ..............70

4.5.Mbp+/- showed alteration of cortical and callosal metabolism ..................................................76

4.6.MBP reduction led to an increase in the number of lba1+ cells in the rostral corpus callosum of Mbp+/- compared with wild-type............................................................................................................78

4.7.Mbp+/- mice specifically showed a decrease in pre-pulse inhibition of startle response and in

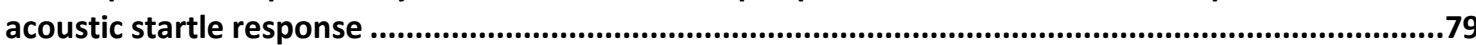

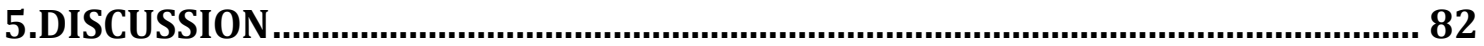

6.TRANSLATIONAL VALIDITY OF THE MODEL AND OUTLOOK FOR FUTURE RESEARCH.................................................................................................. 92

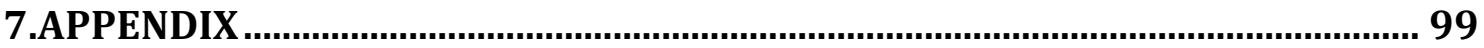

8.LIST OF ABBREVIATIONS................................................................................. 102

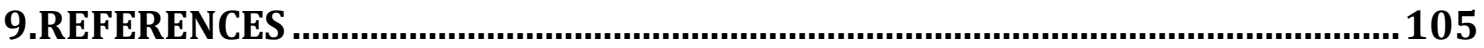





\title{
PREFACE
}

During my PhD I worked on two main projects with the aim of unravelling psychiatric sub-phenotypes. These projects led to the following manuscripts (first authorship):

(1) Cortical network dysfunction caused by a subtle defect of myelination

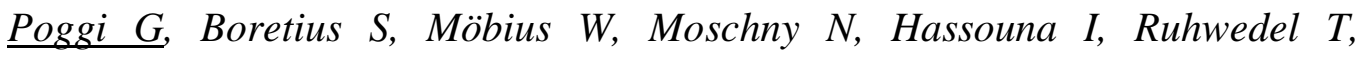
Baudewig J, Nave KA, Ehrenreich H. Manuscript in preparation.

Project: The focus of this project was to understand the role of myelin basic protein reduction in the context of psychiatric disease, specifically schizophrenia. This project is extensively described and discussed in this thesis.

(2) Accumulated common variants in the broader fragile $X$ gene family modulate autistic phenotypes

Stepniak B, Kästner A, Poggi G, Mitjans M, Begemann M, Hartmann A, Van der Auwera S, Sananbenesi F, Krueger-Burg D, Matuszko G, Brosi C, Homuth G, Völzke H, Benseler F, Bagni C, Fischer U, Dityatev A, Grabe HJ, Rujescu D, Fischer A, Ehrenreich H.EMBO Molecular Medicine, in press

\begin{abstract}
Fragile X syndrome (FXS) is mostly caused by a CGG-tripletexpansion in the fragile $\mathrm{X}$ mental retardation 1 gene (FMRl). Up to $60 \%$ of affected males fulfill criteria for autism spectrum disorder (ASD), making FXS the most frequent monogenetic cause of syndromic ASD. It is unknown, however, whether normal variants (independent of mutations) in the fragile $\mathrm{X}$ gene family (FMR1, FXR1, FXR2) and in FMR2 modulate autistic features. Here, we report an accumulation model of 8 SNPs in these genes, associated with autistic traits in a discovery sample of male schizophrenic patients $(\mathrm{N}=692)$ and 3 independent replicate samples: Schizophrenia patients $(\mathrm{N}=626)$, patients with other psychiatric diagnoses $(\mathrm{N}=111)$ and a general population sample $(\mathrm{N}=2005)$. For first mechanistic insight, we contrasted microRNA expression in peripheral blood mononuclear cells of selected extreme group subjects with high- versus low-risk constellation regarding the accumulation model. Thereby, the brain-expressed miR-181 species emerged as potential 'umbrella regulator', with several seed matches across the fragile $\mathrm{X}$ gene family and FMR2. To
\end{abstract}


conclude, normal variation in these genes contributes to the continuum of autistic phenotypes.

I also contributed to other projects that led to the following publications (coauthorship):

(1) Gpm6b deficiency impairs sensorimotor gating and modulates the behavioral response to a 5-HT2A/C receptor agonist.

Dere E, Winkler D, Ritter C, Ronnenberg A, Poggi G, Patzig J, Gernert M, Müller C, Nave KA. Behav Brain Res. 2015 Jan 15; 277:254-63

(2) Fast cerebellar reflex circuitry requires synaptic vesicle priming by munc13-3.

Netrakanti PR, Cooper BH, Dere E, Poggi G, Winkler D, Brose N, Ehrenreich H. Cerebellum. 2015 Jun; 14(3):264-83

(3) The brain as immunoprecipitator of serum autoantibodies directed against the NMDAR subunit NR1

Castillo-Gomez E, Kästner A, Steiner J, Weber F, Hettling B, Poggi G, Asif AR, Pfander V, Gámez Viana MA, Hammer C, Schulz T, Binder L, Stöcker W, Schneider A, Ehrenreich H. Annals of Neurology, 2015 Oct 27. 
PREFACE 


\section{SUMMARY}

Myelin, the proteolipidic layer that wraps around the axon, is one of the evolutionary advantages for animals with complex behaviour and reasoning. Myelin ensures axonal support and synchronised electrical communication among different brain areas. During the last two decades several studies have consistently reported myelin abnormalities in schizophrenia. Myelin Basic Protein (MBP), one of the major myelin structural proteins, has become more and more appealing for the attempt of understanding the role of myelin abnormalities in neuropsychiatric diseases. Due to its essential role in the correct formation of the myelin sheath, we wondered whether a reduction in MBP could be detrimental for the brain connectivity and functionality, hence leading to the appearance of specific symptomatology reported in schizophrenia. We characterised a mouse line expressing only $50 \%$ of MBP, i.e. the heterozygous strain of shiverer mice (here referred as $\mathrm{Mbp}+/-$ mouse). Through the combination of several methods (behavioural testing, magnetic resonance imaging, spectroscopy histology, electron microscopy and biochemical analyses), we proved that, upon ageing,the prefrontal cortex and its underlying white matter are highly sensitive to the lack of one MBP allele. We found reduced pre-pulse inhibition as a translationally valid behavioural readout of mental disease-associated network dysfunction, i.e. sensorimotor gating deficiency. As mechanisms likely contributing to this behavioural abnormality, we detected mild alteration in myelin ultrastructure and brain metabolism in association with microgliosis. Even though it is still unclear how and when the lowered MBP levels in schizophrenia are induced, we show for the first time that MBP reduction by itself does have mental disease relevant consequences 
INTRODUCTION 
INTRODUCTION

\section{INTRODUCTION}

\subsection{Brain white matter: an overview}

The central nervous system (CNS) is conventionally and anatomically subdivided into two main compartments, namely grey and white matter. The grey matter is composed mainly of neuronal cell bodies and dendrites, whereas the white matter, which makes up for roughly $40 \%$ of the human brain, comprises glial cells (astrocytes, microglia and oligodendrocytes) and axons ensheathed by a proteolipidic multilamellar structure, called myelin(Morell 1980). Historically, glial cells were considered solely the "glue" CNS, but now it is clear that this cell sub-population, constituting more than $50 \%$ of the total cell pool in the CNS, contributes to many vital processes in the CNS (Bercury and Macklin 2015).

Astrocytes are the most abundant cell subtype in the CNS. They can be morphologically classified into two groups, i.e. protoplasmic astrocytes and fibrous astrocytes.

Protoplasmic astrocytes are mainly found in the grey matter and form contact with synapses; fibrous astrocytes are typically detected in the white matter and they make contact with the node of Ranvier (Sofroniew and Vinters 2010). Astrocytes have an important role in the clearance of neurotransmitters at the synapse, in the stability of the oligodendrocyte-derived myelin sheath and in the process of myelination (Ishibashi, Dakin et al. 2006, Freeman and Rowitch 2013). Additionally, astrocytes are crucial for the formation of the Blood Brain Barrier (BBB), for the appropriate blood distribution in the CNS and they are often involved in inflammatory response in the injured CNS (Freeman and Rowitch 2013). Astrocytes exhibit electrical activity, favoured by the presence of sodium and potassium channels at the membrane and by intracellular machinerythat is responsive to the increase of intracellular calcium (Sofroniew and Vinters 2010).

Microglia are CNS-specific immune cells, which derives mainly (but not entirely) from the primitive macrophage coming from the embryonic yolk sac (Nayak, Roth et al. 2014). Interestingly, it has been recently showed that in adult mice (when required) microglia can originate from fast proliferation and differentiation of nestin-postive cells (Elmore, Najafi et al. 2014). In physiological condition, microglia present a highly 
ramified morphology, with small soma and fine cellular processes, and they keep extending and retracting their processes to scan the CNS environment. In case of injury in the CNS, microglia changeto aamoeboid morphology, with short and thick processes, reorganise the surface molecules and release immunoregulatory or pro-inflammatory components (Orr, Orr et al. 2009, Kettenmann, Hanisch et al. 2011).

On top of the immune surveillance task, microglia participate to promoting neuronal survival, synaptogenesis, myelin maintenance and re-myelination(Miron, Boyd et al. 2013, Nayak, Roth et al. 2014).

Oligodendrocytes are the cells dedicated to the formation of myelin in the CNS. An oligodendrocyte could myelinate up to 50 axons, ensuring (1) the proper electrical conduction along the axon and (2) an appropriate axonal support (Baumann and PhamDinh 2001, Simons and Trotter 2007)

\subsection{Myelin and myelination in the CNS}

\subsubsection{0ligodendrocytes precursor cells and mature oligodendrocytes}

Oligodendrocytes originate from the maturation of neural stem cells-derived Oligodendrocyte Precursor Cells (OPCs). These precursors, identified by the expression ofplatelet-derived growth factor receptor (PDGFR)-alpha and neural/glial antigen (NG) 2 markers, can arise from the ventral and dorsal embryonic spinal cord and from the ventral and dorsal forebrain, depending on the stage of development (Richardson, Kessaris et al. 2006, Bercury and Macklin 2015). The spatiotemporal distinction of OPCs is also accompanied by differencesin the expression of transcription factor, which appears to be specific to each OPC subpopulation (Chong and Chan 2010). Part of the OPCs remains in the precursor stage. These precursors reside in the CNS as a potential reservoir of undifferentiated cells for the adult CNS.If required,they can be pushed to maturation at later stages, for instance, to support adult myelination(Chong and Chan 2010). While a pool of OPCs remains in the precursor stage, another pooldevelops towards the status of mature oligodendrocyte already at early stage of the CNS development (Nishiyama, Komitova et al. 2009). The process of maturation requires changes in morphology, in molecular machinery and in surface markers of the OPC (Fig.1) 


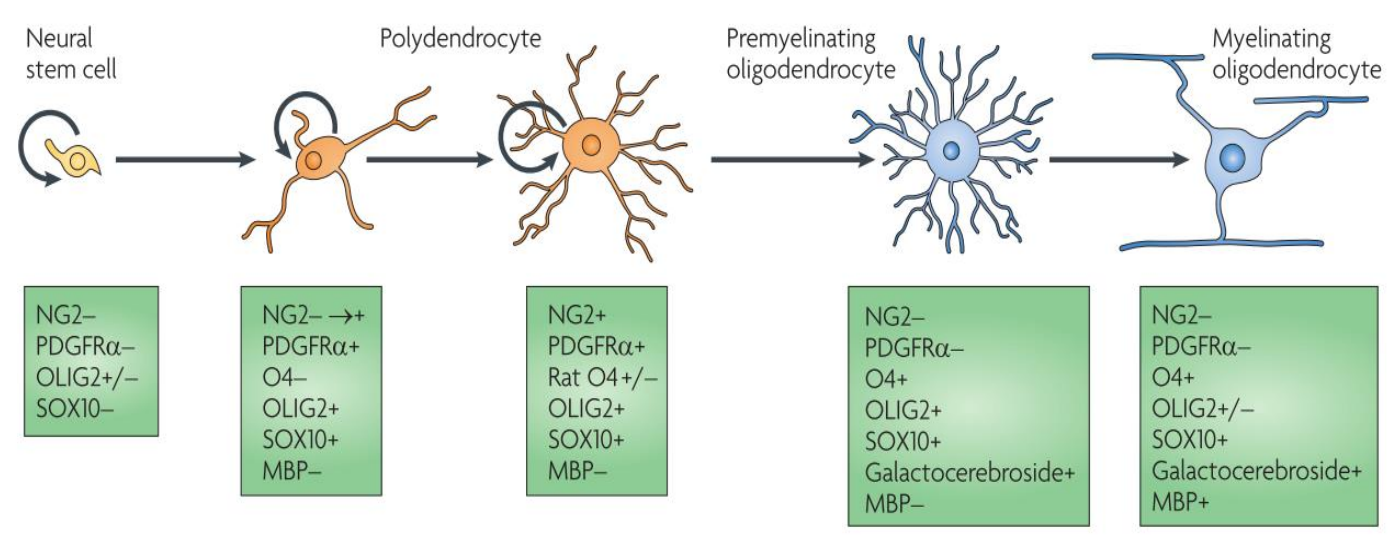

Figure 1.Developmental stages from neural stem cell to mature oligodendrocytes. NG2 and PDGFR $\alpha$ are absent in neural stem cells, but they are specifically expressed in proliferating (proliferation $=$ semicircular arrows) progenitor cells. Polidendrocytes (commonly accepted as OPCs) are rich in processes, whose size and number are spatiotemporally regulated. Upon differentiation OPCs stop expressing NG2 PDGFR $\alpha$ and start expressing mature oligodendrocyte molecules, like myelin basic protein (MBP).

Figure from (Nishiyama, Komitova et al. 2009)

Extrinsic and intrinsic signals regulate proliferation, migration and differentiation of OPCs. For instance, it has been shown that overexpression of PDGF by neurons induces OPC proliferation (Chong and Chan 2010). Furthermore, electrical activity, contact with axons and glutamate signalling can influence OPCs proliferation, migration and differentiation (Boiko and Winckler 2006, Gudz, Komuro et al. 2006). This highlights the importance of the communication between OPCs and neurons for the proper formation and functioning of the CNS, and for the proliferation and differentiation of OPCs (Gudz, Komuro et al. 2006, Chong and Chan 2010).However, even in the absence of external cues, OPCs can still follow an intrinsic program of proliferation and maturation, suggesting that OPCs have an intrinsic timer that regulates their fate(Chong and Chan 2010).

Upon the combination of extrinsic and intrinsic cues, OPCs progress towards the mature status. In this phase, the Oligodendrocyte Transcription Factor 1(Olig1) contributes actively to the transcription of several myelin-related genes, i.e. Myelin Basic Protein(Mbp), Proteolipid Protein (Plp) and Myelin Associated Glycoprotein (Mag) genes (Xin, Yue et al. 2005, Dai, Bercury et al. 2015).

Mature oligodendrocytes originate the multilamellar lipoproteic ensheathment of CNS axons, i.e. CNS myelin. In the human CNS, myelin wraps around axons of at least $1 \mu \mathrm{m}$ 
diameter (Sherman and Brophy 2005), whereas in the mouse corpus callosum myelin can be detected around 0.2 $\mu \mathrm{m}$-diameter axons (Waxman and Swadlow 1976, Sturrock 1980).

\subsubsection{Myelin biogenesis: processes elongation and axon ensheathment.}

As anticipated, myelin derives from the growth and protrusion of oligodendrocyte processes that make contact and wrap concentrically around axons at periodically discontinuous points, namely the internodes. The myelinated internodes - about $150 \mu \mathrm{m}$ in length - alternate with the node of Ranvier, where sodium ion channels cluster instead (Deber and Reynolds 1991, Sherman and Brophy 2005). Once the OPCs reach their destination, they start extruding and retracting their processes, scanning the surrounding environment. The myelination process begins when the tip of an OPC process establishes contact with the axon to myelinate. Axon diameter and integrin-mediated axon-glia interaction seem to be important factors for an OPC to selectively start myelination (Camara, Wang et al. 2009, Lee, Leach et al. 2012). Moreover, it has recently been shown in zebrafishthat neuronal activity works as a cue for OPCs specification and as a signal forstabilising the glial-axon contact for myelination(Petersen and Monk 2015). Nevertheless, the complete molecular mechanism involved in the selection of a specific axon and in the stability of the gliaaxon connection is not fully understood. Even if the molecular players are not disclosed yet, it is known that such contact creates a commitment between axon and OPC, stimulates the polarisation of the OPC molecular machinery towards the axon-glia interaction site, and induces the consequent evolution of the OPC towards myelinating oligodendrocyte status (Simons and Nave 2015). The biogenesis of the myelin membrane requires extreme extension of the oligodendrocyte processes; a rough estimation of the oligodendrocyte processes growth in rat brain during myelination revealed that the membrane surface area increases at a rate of $5-50 \times 10^{3} \mu \mathrm{m}^{2} /$ cell/day (Baron and Hoekstra 2010). Additionally, specific sorting of myelin components is required to ensure the proper assembly of the myelin sheath. To fulfil these demands, myelin components are assembled in proteolipidic domains (lipid rafts) in the Golgi apparatus and then delivered via non-targeted vesicular transport to the myelinating processes. A change in the balance of endo-/exocytosis pathway is speculated to be a crucial step to increase the transport rate of membrane component to the 
oligodendrocyte plasma membrane, whereas endocyticrecycling, feature of maturingoligodendrocytes, seems to ensure spatiotemporal specificity in the myelin biogenesis and local membrane remodelling (White and Kramer-Albers 2014). In the process of endocytic recycling, myelin proteins in the plasma membrane are reinternalised and directed to endocytic compartments; from there the proteins are then sorted to specific domains in the plasma membrane (Fig. 2)(Maxfield and McGraw 2004, Winterstein, Trotter et al. 2008).

It is worth mentioning that neuron-to-glia signalling regulates the endocytic sorting and trafficking of PLP, underlying once more the importance of neuron-glia communication for the process of myelination (Simons and Trotter 2007, Winterstein, Trotter et al. 2008, White and Kramer-Albers 2014).

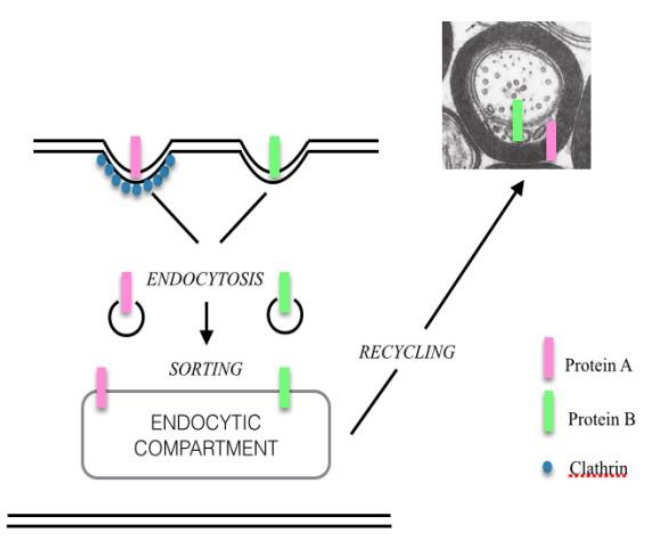

Figure 2.Myelin proteins trafficking and membrane remodelling.Myelin proteins can be internalised via clathrindependent or clathrin-independent endocytosis. They are sent to the endosomal compartment (dependent on the protein) and from there they are sorted and recycled to the appropriate myelin domain.

Figure adapted from(White and KramerAlbers 2014)

How oligodendrocyte processes can finally wrap around the axons and originate the compact myelin structure has been subject of discussion for several years. So far, few models have been proposed.

The first suggested model, later defined as Carpet crawler, derived from a study in cat spinal cord. The author claimed that oligodendrocyte processes would entirely cover the axonal area - that would later become the internode - before making the first turn. The ensheathment would proceed until myelin had reached the appropriate thickness (Bunge, Bunge et al. 1961).

However, ultrastructural observations of the growing myelin sheath revealed clear inconsistency with this model.In particular, the different layers of growing myelin often show variable length all along the internode (Knobler, Stempak et al. 1976), which is 
physically incompatible with the suggestion that oligodendrocyte processes would entirely cover the future internode since the first wrap.

The more recent liquid croissant model tried to explain the contradiction of the previous prototype.

In this model the oligodendrocyte process - reminiscent of a dough edges of a croissant - would be "poured out" in a triangular shape and, under the guidance of the axonal proteins, it would spread along the future internode (Sobottka, Ziegler et al. 2011).

The most updated model of myelin wrapping was published in 2014. Via the combination of in vivo live imaging and ultrastructural analysis, it was possible to confirm that myelin formation occurs through the wrapping of the triangular-shaped inner tongue (leading edge) around the axon. The "wrapping movement" is also accompanied by a lateral growth of each individual myelin layer. This model also shed light on the route of communication between the leading edge and the axon. In fact transient cytoplasmic channels, regulated by increase in phosphatidylinositol-(3,4,5,)triphosphate (PIP3), are open during myelin growth, as a shortcut to transport myelin components to the leading edge. Cytoplasmic channels are normally closed after completion of the myelin growth. Interestingly, in adult mice they could be reopened in case of PIP3 increase, leading to a consequent increase in myelin thickness (Snaidero, Mobius et al. 2014). This feature indicates that myelin is a dynamic structure that maintains its plasticity also in adulthood.

\subsubsection{Myelin structure}

Myelin has a unique composition that distinguishes it from all the other biological plasma membranes. In vivo, the water content of myelin is around $40 \%$ and the dry weight of myelin is composed of $70-80 \%$ of lipids and $20-30 \%$ of proteins, which ensure to myelin a very high lipid-to-protein ratio (around 0.25) (Quarles 2006, Aggarwal, Yurlova et al. 2011, Aggarwal, Yurlova et al. 2011). In particular, myelin is rich in glycosphingolipids (about $27 \%$ of the total lipid content in the form of galactosylceramide and sulfatide), plasmalogens (about $16 \%$ of the total lipid content) and cholesterol (crucial for defining the fluidity of the myelin sheath) (Quarles 2006, Aggarwal, Yurlova et al. 2011, Aggarwal, Yurlova et al. 2011). Galactosylceramide, together with its sulfatated and hydroxylated forms, is relevant for the interaction of 
oligodendrocytes and axons at the paranodes and for the correct organisation of the $\mathrm{Na}+$ channel at the node of Ranvier; plasmalogens seem to protect the myelin sheath from oxidative stress; cholesterol was proven to be a rate-limiting factor for myelin production (Jahn, Tenzer et al. 2009, Saher, Quintes et al. 2011). This high lipid composition limits the passage of ions through the myelin sheath, ensuring electrical insulation (Fitzner, Schneider et al. 2006, Chrast, Saher et al. 2011).

The protein composition of the CNS myelin is also very peculiar, with only three members accounting for the major proportion of myelin-related proteins, i.e. PLP (30$54 \%$ of the total proteins), MBP (22-35\% of the total proteins) and 2':3'-Cyclic nucleotide 3'-phosphodiesterase (CNP, 4-15\% of the total proteins) (Jahn, Tenzer et al. 2009). These proteins specifically occupy defined compartments of the myelin structure. Ultrastructural observations of a myelinated axons (cross-sectionally and longitudinally) via Electron Microscopy (EM) reveals two main domains of the myelin sheath: the compact myelin and the non-compact myelin (Fig. 3).

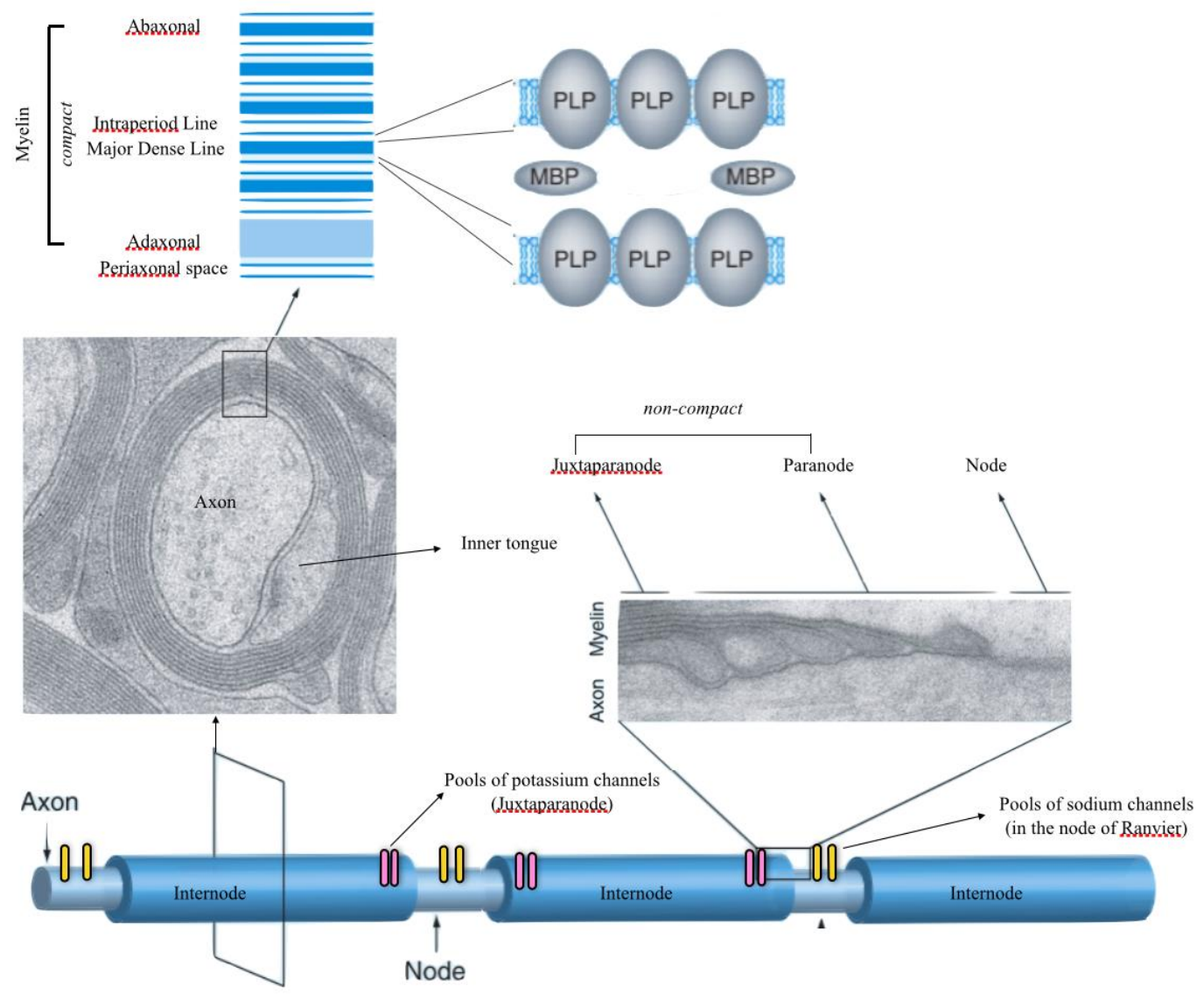

Figure 3.Representation of the myelin structure with the major myelin proteins.

The cartoon shows a myelinated axon with three internodes and the representative distribution of sodium and potassium channels. EM images offer cross-sectional (left) and a longitudinal (right) view, respectively of the internode and of the paranode.

The cartoon (top) shows the different layers of the myelin sheath surrounding the axon (left), with the main proteins of the compact myelin (right).

Figure adapted from (Werner and Jahn 2010) 
These two domains differ in morphology, function and protein composition (Quarles 2006, Simons and Nave 2015).

The compact myelin consists of tightly apposed oligodendrocyte plasma membranes. In this domain, the compaction of the intracellular surface of the plasma membrane extrudes the cytosol, originating the so called Major Dense Line (electron-dense layer in the EM micrographs); the apposition of the extracellular surface of the oligodendrocytes plasma membrane gives rise to the so called Intraperiod Line (electron-light layer in the EM micrographs) (Quarles 2006). The protein composition of the compact myelin is less heterogeneous than the one of the non-compacted myelin, yet this compartment contains the most abundant proteins of myelin, i.e. PLP/DM20 and MBP.

PLP gene encodes two main protein isoforms: PLP and DM20, which differ only for 35 additional amino acids at the cytosolic extremity, missing from DM20.

PLP is a tretraspanning protein necessary for the formation of the Intraperiod Line of myelin. Although the structural similarity with DM20 would point in the direction of a comparable functionality of the two proteins, it is still unclear whether DM20 could make up for the absence of PLP in myelin compaction (Quarles 2006, Fulton, Paez et al. 2010). Beside the structural function, PLP seems to be involved in the exchange of ions across the oligodendrocytes plasma membrane and in the signal transduction between extracellular matrix and intracellular space of the oligodendrocytes. Additionally, some evidences propose a role for PLP in the maturation and migration of OPC and in the oligodendrocyte survival, via influence on the apoptotic cascade and on the mitochondria function(Quarles 2006, Fulton, Paez et al. 2010). PLP seems also to be involved in the myelin biogenesis, via binding to lipid rafts cholesterol; the delivery of PLP from late endosome to the plasma membrane via exocytosis seems to be promoted by signalling from neurons (Quarles 2006, Trajkovic, Dhaunchak et al. 2006, Fulton, Paez et al. 2010, White and Kramer-Albers 2014). MBP is the second most abundant protein of the myelin sheath and it mediates the apposition of oligodendrocyte intracellular surfaces to drive myelin compaction. Its function will be more extensively explained in section 2.2.4.

The non-compacted myelin - likely the most metabolically dynamic - comprises all the area of the myelin sheath where the cytoplasm of the myelinating oligodendrocyte has not been completely extruded via the process of compaction, namely the inner-tongue (in intimate contact with the axon), the outer-tongue (most distant from the axon), the 
juxtaparanode (most likely mediating axon-glial communication and maintaining neuronal resting potential via voltage-gated potassium channels) and paranodal loop (in proximity of the nodes of Ranvier) and the cytoplasmic channels, that ensure fast exchange of newly synthesised membrane components from the oligodendrocyte soma to the leading edge during the process of myelination (Snaidero, Mobius et al. 2014)(Poliak and Peles 2003). The non-compacted myelin contains several different proteins in low abundance, like Myelin Associated Glycoprotein (MAG), Sirtuin 2 (Sirt2) and among them CNP is one of the most abundant (Nave and Werner 2014). CNP is also expressed in other cell types, like neurons and microglia, but is much more prominent in the CNS myelin (Quarles 2006, Yang, Kan et al. 2014). It is present in two different isoforms $(46 \mathrm{kDa}$ and $48 \mathrm{kDa})$. Both these isoforms undergo specific posttranslation modifications, namely acylation and isoprenylation at the C-terminus, which promotes their association to membrane components(Quarles 2006). CNP is fundamental for myelin-axon physiology. In mice lacking the CNP protein myelin appears normal, but the myelin-axon interface is affected, leading to axonal swelling and neurodegeneration (Lappe-Siefke, Goebbels et al. 2003). CNP is highly relevant for the organisation of the node of Ranvier(including the distribution of the $\mathrm{Na}^{+}$channels) and for the proper timing of the myelin compaction, acting almost in antagonism to MBP. In fact,it has been reported that the absence of CNPcan significantlyspeed up the apposition of oligodendrocyte intracellular surfaces, mediated by MBP (Fulton, Paez et al. 2010, Aggarwal, Yurlova et al. 2011, Snaidero, Mobius et al. 2014). Additionally, CNP interacts with cytoskeleton, RNA and calmodulin, suggesting a broader range of function of CNP in the brain (Myllykoski, Seidel et al. 2015).

\subsubsection{The "classical" MBP}

MBP family is transcribed from a $>100 \mathrm{~kb}$ gene complex, known as golli (genes of oligodendrocytes lineage). Golli is located on chromosome 18, in both human and mouse, and the exonic sequences are highly conserved between the two species (Pribyl, Campagnoni et al. 1993). The golli gene presents three transcription start sites, that code for the classic MBPs and the golli MBPs(Fig.4).

The golli MBPsare the products of the first transcription start site and they can be detected in several brain cells and other organs. Depending on the cell type, golli 
MBPscan perform several various functions, ranging from contribution to cell migration to intracellular $\mathrm{Ca}^{2+}$ regulation (Fulton, Paez et al. 2010).

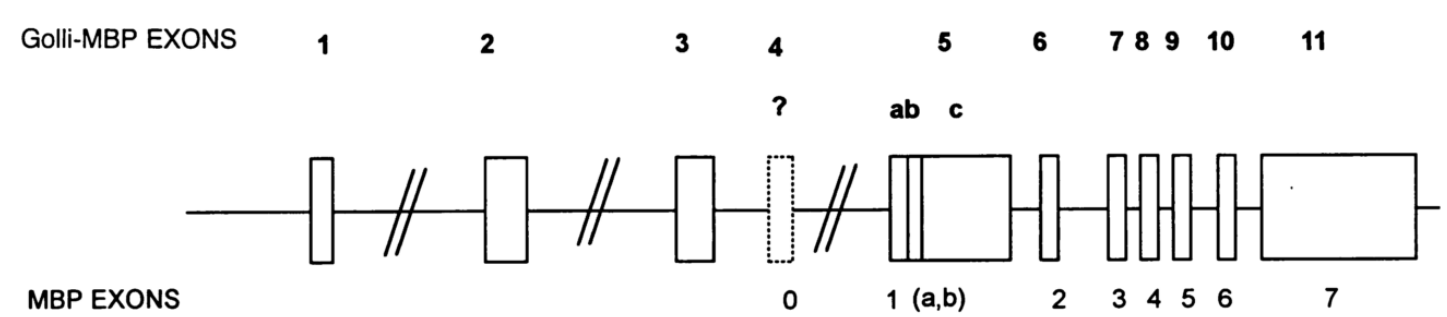

Figure 4.The golli-gene. The golli-gene contains exons that code for the golli-MBPs and for the classical MBPs.

Figure from (Pribyl, Campagnoni et al. 1993).

The classical MBPs (referred as MBP in the next pages) are mainly the products of the transcript that originates from the third transcription start site of the golli gene (7 exons). These products give rise to several sized isoforms; ranging from $14 \mathrm{kDa}$ to $21.5 \mathrm{kDa}$ in the mouse and from 17.2kDa to 21.5kDa in humans (Boggs 2006).

Once the $M b p$ mRNA is transcribed, it assembles in granules in the soma of the oligodendrocyte and, thanks to the kinesin motor protein Kinesin Family Member (kif) $1 \mathrm{~b}$, it is anterogradely transported on microtubules to reach the more distal extremity of the oligodendrocyte processes (the leading edge during myelination). Here it is translated and the protein is directly inserted between the cytoplasmic leaflets (Ainger, Avossa et al. 1997, Harauz, Ladizhansky et al. 2009).

The appropriate translocation of the $M b p$ mRNA is mainly favoured by (1) RNA binding molecules that recognise responsive elements on the $M b p$ mRNA (Heterogeneous nuclear ribonucleoprotein - hnRNP) and by (2)RNA Localisation Region (RLR) at the 3'UTR of Mbp (Ainger, Avossa et al. 1997, Muller, Bauer et al. 2013).

The translation of $M b p$ is prevented during the mRNA translocation via a repressive mechanism, mediated by small non coding RNA and RNA binding protein(White, Gonsior et al. 2008, Muller, Bauer et al. 2013). Axon-glial contact at the extremity of the oligodendrocyte processes inhibits this repressive mechanism to ensure a spatiotemporally regulated translation of Mbp (White, Gonsior et al. 2008, Muller, Bauer et al. 2013). In vitro electrical activity of the axons has also been reported as a stimulating factor for $M b p$ translation (Wake, Lee et al. 2011). The product of the 
Mbptranslation includes several intrinsically unstructured splice variants, whose expression is temporally and spatially regulated too. Exon II-containing isoforms giving rise to $21.5 \mathrm{kDa}$ and $17.2 \mathrm{kDa}$ proteins - are mainly expressed during development; compared with the exon-II missing isoforms, they present 26 additional amino acids that most likely make them preferentially targeted to the cytosol and to the nucleus of oligodendrocyte(Allinquant, Staugaitis et al. 1991, Harauz, Ladizhansky et al. 2009). Exon II-missing isoforms - originating $14 \mathrm{kDa}$ and $18.5 \mathrm{kDa}$ proteins - gather at the oligodendrocyte plasma membrane instead (Allinquant, Staugaitis et al. 1991).

Due to several post-translational modifications (i.e. deimination, phosphorylation, deamidation, methylation, and N-terminal acylation), each of the exon II-missing isoform appears in nature in differently charged isomers (Fig.5). This condition adds a further level of complexity to MBP and implies that, most likely, each one of the isomers has a different function and binding capability in the context of myelination (Moscarello 1997, Boggs 2006, Harauz, Ladizhansky et al. 2009).

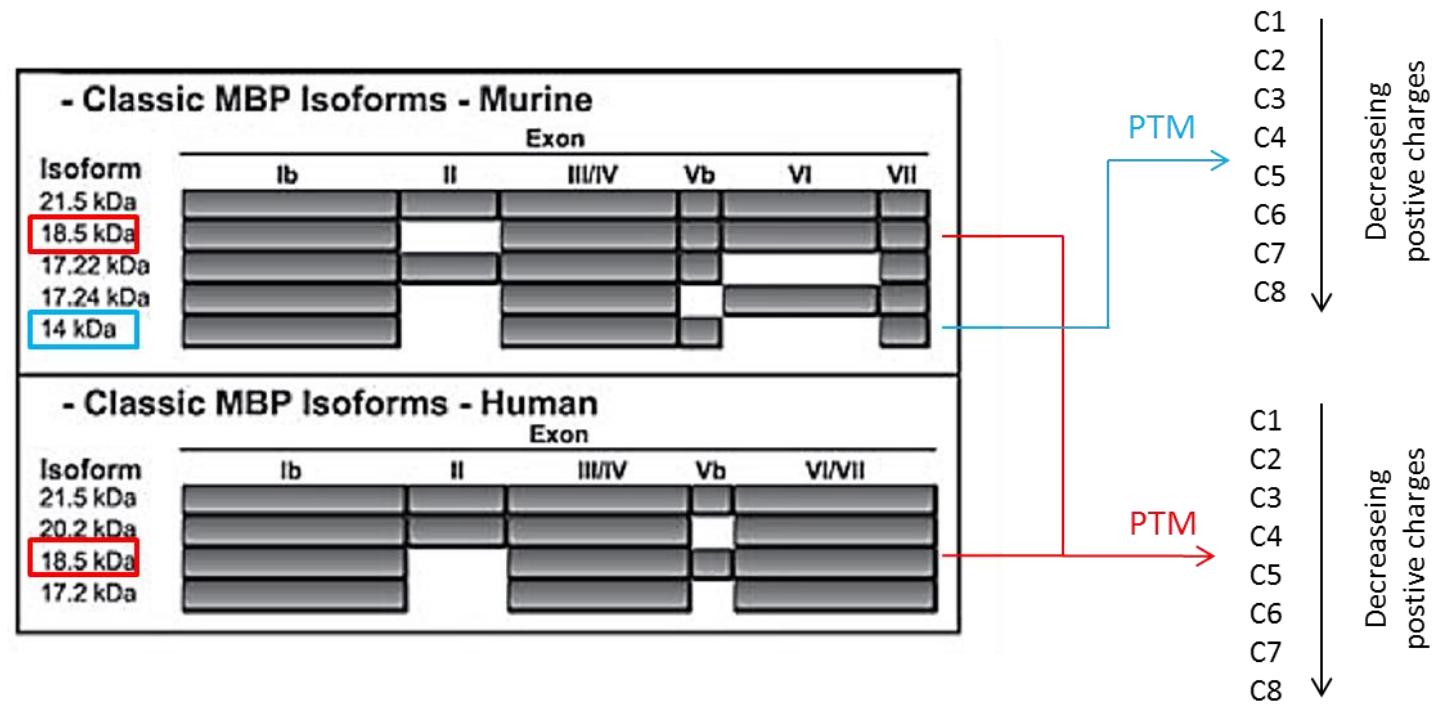

Figure 5.The MBP family. Several different charged isomers and isoforms derived from the MBP gene. Alternative splicing and post-translational modification (PTM) originate a broad range of MBP molecules. Each one of these isomers/isoforms could have a specific function that does not share with the other isoform in the context of myelination and myelin compaction.

Figure adapted from (Moscarello 1997, Boggs 2006)

MBP performs several crucial functions in the proper assembly of the CNS myelin. Due to the positive charges, MBP binds the negative charges of the plasma membrane lipids and it has a leading role in apposing the intracellular side of the oligodendrocyte plasma 
membrane to form compact myelin around axons. Specifically, MBP stably interacts with phosphatidylinositol 4,5-bisphosphate (PIP2) and pulls together the oligodendrocyte plasma membrane to form the Major Dense Line. Interfering with MBP-PIP 2 binding can be detrimental for the CNS myelin (Nawaz, Kippert et al. 2009). MBP is also a major player in the oligodendrocyte polarisation and in the organisation of myelin components. It has been showed that, following oligodendrocyte interaction with an axon, MBP influences the lateral organisation/clustering of lipids in the oligodendrocyte plasma membrane. This organised lipid distribution is lost in oligodendrocyte from Shiverer mouse model (lacking both $M b p$ alleles (Fitzner, Schneider et al. 2006). Additionally, MBP has a pivotal role in the mechanical extrusion of cytoplasm and proteins with large cytosolic domain from the myelin sheath, literally "zippering them out", while pulling together the cytoplasmic side of the oligodendrocyte plasma membrane (Aggarwal, Yurlova et al. 2011). This sieve-like

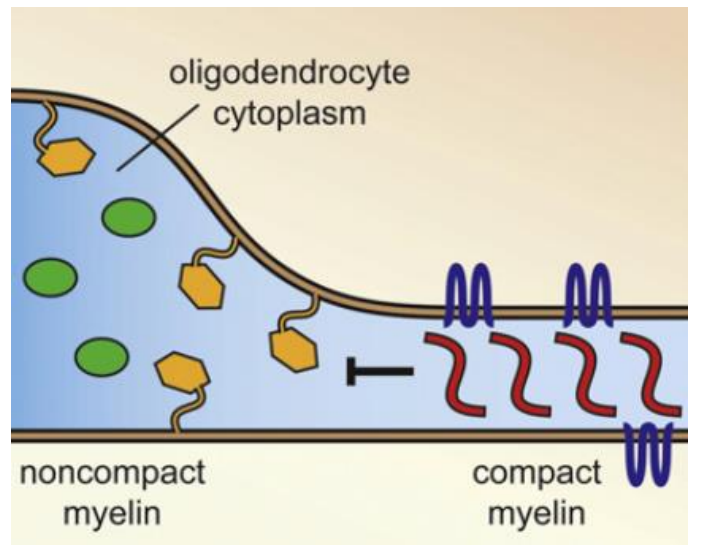

$\sum$ MBP M $M_{\text {protein }}^{\text {compact myelin }}$

noncompact myelin, membrane-bound protein

Figure 6.Sieve-like behaviour of MBP.MBP zippers out the cytoplasm and the proteins with large cytosolic domain leading compaction of the myelin sheath. Figure from (Zuchero and Barres 2011) behaviour of MBP ensures the maintenance of the high lipid-to-protein ratio and selectively defines which proteins can enter the myelin sheet (Fig. 6) (Aggarwal, Yurlova et al. 2011).

Additionally, MBP binds in vivo to tubulin (Harauz, Ladizhansky et al. 2009) and several works demonstrated the ability of MBP to interact in vitro with actin filaments at the leading edge during myelin sheath formation. The binding to actin filamentswas speculated to be a hint on the participation of MBP also in the membrane extension and wrapping (Boggs 2006, Boggs, Rangaraj et al. 2011). Indeed recent publications suggest that actin disassembly is necessary for myelin wrapping and it

has been proposed that MBP competes with protein involved in actin disassembly to bind to PIP2. This would displace (and consequently activate) "actin disassembly proteins", indirectly boosting myelin wrapping (Zuchero, Fu et al. 2015). 
For its disorganised chemical structure it is believed that MBP could adapt to many more different interaction partners, which makes this protein probably one of the most crucial, yet not totally understood, molecules of the CNS.

\subsection{The relevance of myelin and myelination}

Rapid and synchronised electrical conduction along axons is a priority in animals with sophisticated behaviour. To facilitate electrical conduction, evolution has offered the ensheathment of axons by myelin, whose thickness is proportionally dependent on the axon diameter. Due to this dependence, myelin thickness is estimated via g-ratio, the index derived from the ratio of the axonal diameter to the total outer diameter (i.e. axon diameter + myelin diameter). Theoretical estimation of the optimal g-ratio predicted values ranging from 0.6 to 0.7 , however these values do not apply to all the myelinated nerve fibres of the nervous system (Purger, Gibson et al. 2015).

Myelin wraps around almost the whole length of the axon, leaving uncovered only the nodes of Ranvier. As insulating membrane, myelin reduces the transverse capacitance between the intra- and extra-axonal environments, it increases the transverse resistance of adjacent internodes and it reduces axonal membrane capacitance. In this way, electrical current flows from a node of Ranvier to the next, without dissipating, leading to 20-100-fold faster nerve saltatory conduction (Hartline and Colman 2007, Nave and Werner 2014). The fine tuning of neuronal synchrony could be promoted by change in the conduction velocity through modification of myelin thickness (Pajevic, Basser et al. 2014).

Myelin is not only orchestrating electrical conductivity, but it has been recently reported to be pivotal for metabolic and trophic support of the axons. For instance, vesicles transfer from oligodendrocytes to axons could be a route for transferring support molecules(Kramer-Albers, Bretz et al. 2007, Fruhbeis, Frohlich et al. 2013). Moreover, mature oligodendrocytes produce lactate and pyruvate via aerobic glycolysis (Funfschilling, Supplie et al. 2012). The lactate is supplied to axons through a glial lactate transporter and axons metabolise it when other energy sources are not available. The inhibition of the lactate transporter induces clear axonal damage, highlighting the 
intimate dependence of the axon to the myelin sheath (Lee, Morrison et al. 2012, Nave and Werner 2014).

Is then myelin a passive component that ensures viability and functionality of the axons? No, myelin is more than that. Myelin is not a static structure that wraps around axons only to ensure conductivity and metabolic support. Myelin starts developing early in life both in human and rodents; while rodents myelination reaches its peak around post-natal day 23 (P23)(Foran and Peterson 1992)in human the development continues through adulthood, reaching the maximum peak around middle age (Miller, Duka et al. 2012).Moreover, both in human and in rodents, white matter content reduces in physiological ageing (Sturrock 1987, Haroutunian, Katsel et al. 2014). Myelin is a dynamic ensheathment that also changes in response to axonal and environmental stimuli (Bechler and ffrench-Constant 2014). The mechanisms regulating myelin plasticity have just started being disclosed. For instance, as already mentioned in the previous sections, in vitro electrical activity of the axons has a direct influence on the translation of MBP (Wake, Lee et al. 2011), and it inhibits OPC proliferation to promote differentiation (Stevens, Porta et al. 2002) and consequent myelination. This suggests that changes in electrical activity can directly promote myelin formation. On the other hand, blockade of the action potential has been proven to reduce OPC proliferation and cause hypomyelination (Barres and Raff 1993, Demerens, Stankoff et al. 1996).

In the orchestration of in vitro myelin formation, different frequencies of electrical stimulation could have opposite effects (Stevens, Tanner et al. 1998), pointing out how myelination plasticity is not only a numb reaction to electrical activity, but the elegant response to a defined and specific electrical stimulus.

In vitro studies find further support from in vivo experiments. In zebrafish, neuronal activity and synaptic vesicles release influencethe process of myelination (Hines, Ravanelli et al. 2015, Mensch, Baraban et al. 2015). Optogenetic stimulation of the cortical layer $\mathrm{V}$ in wild-type mice leads to an increase of OPC proliferation and differentiation and a consequent decrease in myelin g-ratio; interestingly, these cellular changes underlie the acquisition of a complex motor phenotype, suggesting that myelin plasticity and behavioural performances are strictly interconnected (Gibson, Purger et al. 2014).

Positive and negative environmental stimuli also profoundly affect CNS myelination.

For instance, alteration in the sleep pattern alters myelin structure and forced sleep deprivation can be detrimental on myelination; in fact, mice undergoing normal sleep 
report higher rate of OPC proliferation than mice subjected to forced sleep deprivation (Bellesi, Pfister-Genskow et al. 2013). Sleep has been described as "the price we pay for plasticity" and during sleep we consolidate and integrate memory (Tononi and Cirelli 2014), so it appears that myelin exerts its plastic adaptation to environmental stimuli even during sleep.

As much as forced sleep deprivation alters myelin, also stress and glucocorticoids have been reported to change oligodendrogenesis in rat hippocampus (Chetty, Friedman et al. 2014). Moreover, it has been observed that social and sensory deprivation can reduce myelin thickness and myelin-related proteins in mice (Liu, Dietz et al. 2012, Makinodan, Rosen et al. 2012, Barrera, Chu et al. 2013). Intriguingly, such impairment in myelination can have long-term consequences only if the negative environmental experience takes place during "developmentally critical periods" (P21-P35) (Liu, Dietz et al. 2012, Makinodan, Rosen et al. 2012, Barrera, Chu et al. 2013). Not surprisingly, enriched environment can, on the other hand, promote myelination in rats (Yang, Li et al. 2013) and it has also recently showed that OPC and myelination are crucial for mice to learn complex motor tasks (McKenzie, Ohayon et al. 2014)and motor learning per se can increase white matter in rats (Sampaio-Baptista, Khrapitchev et al. 2013).

The discoveries in rodents are in line with human data. Extensive learning of specific motor tasks has been associated with increase in white matter content(Bercury and Macklin 2015).

Learning how to read and improving cognitive functions correlate with white matter development in children (Fields 2005) and even Albert Einstein's intelligence has been correlated to the size of his corpus callosum, highly myelinated area of the brain(Men, Falk et al. 2014).

Furthermore, the capability of learning non-native speech sounds also correlates with white matter size, and social cognition (acquired during adolescence) develops in parallel to an increase in white matter density in the frontal area of the brain (Paus 2005).

Not surprisingly, adaptiveness of myelination is a double-edge sword. Being on the one hand a footprint and an irreplaceable requirement for high cognitive and social functions, on the other hand, myelin is extremely sensitive to insults.Expectedly, any small abnormality in the proper myelin wrapping could lead to biological and behavioural alteration with major consequences. 


\subsection{Myelin abnormalities in schizophrenia: focus on MBP}

\subsubsection{Schizophrenia}

Schizophrenia is a dramatic and debilitating psychiatric disorder, presenting a lifetime risk in the order of $0.7-1 \%$ in the overall world population (van Os and Kapur 2009, Kas, Kahn et al. 2011). Men are slightly more likely to develop schizophrenia and they tend to show an earlier onset of the pathology (Aaron T. Beck 2011). The impact is detrimental both on the quality of life of patients and their family - often subjected to public stigma (Corrigan and Watson 2002) - and it is a burden on the health care system economy (Insel 2010). The disease is generally diagnosed in the late adolescence/early adulthood (Walker, Kestler et al. 2004), and it is usually preceded by signs of behavioural dysfunctions (prodromal phase), like reduced tolerance to normal stress, higher emotionality and alteration in body perception (Klosterkötter, Hellmich et al. 2001).However, slight delay in developmental milestones (i.e. walking) and nonspecific emotional and behavioural disturbances are sometimes detectable already during childhood in individuals who later develop the pathology (Welham, Isohanni et al. 2009).

Schizophrenia is characterised by a broad spectrum of symptoms which could be subdivided in "Positive Symptoms" (hallucinations, thought disorders, delusions), treatable with the current available antipsychotic, "Negative Symptoms" (social withdrawal, loss of affect) and "Cognitive impairment-related Symptoms", both significantly less responsive to the standard treatments (Walker, Kestler et al. 2004, Aaron T. Beck 2011).

The aetiology of schizophrenia is not clear, but twin/family studies have proven that there is a genetic contribution to the pathology (Sullivan, Daly et al. 2012). However the concordance rate in monozygotic twin studies is only $48 \%$, indicating that the genetic input to the disorder is only partial and that non-genetic factors (i.e. environment and gene-environment interaction) have a crucial influence on the development of schizophrenia (van Os, Kenis et al. 2010, Sullivan, Daly et al. 2012).

The gene-environment interaction and the complexity of schizophrenia have become even more evident in the last decade, when genome-wide association studies(GWAS) have replaced linkage studies in the field of schizophrenia research. Case-control studies of substantial size (around 20000 individuals per group), comparing healthy individuals versus schizophrenic patients, have solidly predicted several candidate genes for 
schizophrenia(Consortium 2011). However, as expected, the index of the strength of the association between the genotype and the disease, defined as odds ratio $(\mathrm{OR})$, ranges between 1.04 and 1.26(Consortium 2011). These results underlie a minimal contribution of each genetic factor to the disorder.Moreover they raise the hypothesis that genes by themselves are not sufficient to cause the pathology and that environmental and geneenvironment interaction influences are also involved in the aetiology of schizophrenia (van Os, Kenis et al. 2010). Furthermore, the results from GWAS have indirectly pointed out other two important factors to understand schizophrenia. First of all, we could expect unexpressed genetic vulnerabilities for schizophrenia to be common in the general population, further justification for the low OR. Secondly, it is also plausible that specific genetic risk factors could contribute to defined sub-phenotype of the disease and not to the overall pathology (Walker, Kestler et al. 2004).

Several biological mechanisms have been involved in schizophrenia and uncountable "hypothesis of schizophrenia" have been proposed. Structural (loss of grey matter, alteration of white matter, increase ventricles size) and functional changes seem to affect multiple areas of the brain in schizophrenia patients(Buckley 2005, Gur and Gur 2010). Experimental evidences reported alteration of neurotransmitters (dopamine, gamma-Aminobutyric acid -GABA- , glutamate, and serotonin) and their receptors/transporters(Ross, Margolis et al. 2006, Howes, McCutcheon et al. 2015, Spies, Knudsen et al. 2015). Inflammation and increase in inflammation markers have also been consistently associated with schizophrenia (Fillman, Cloonan et al. 2013, Khandaker, Cousins et al. 2015). Furthermore, altered adult neurogenesis (Reif, Schmitt et al. 2007), abnormalities in synaptic pathway, in connectivity and in neural synchrony (Uhlhaas and Singer 2010, Hall, Trent et al. 2015) have been disclosed in schizophrenia patients. Even faster telomere erosion has been reported in schizophrenia patients (Kao, Cawthon et al. 2008).

Reconcile all these different discoveries is not trivial. Consequently, in order to reduce such genetic and phenotypical complexity, it is helpful to focus on a more basic/elementary phenotypes common to several schizophrenia cases (Gottesman and Gould 2003). In the last decades, due to the improvement of the Magnetic Resonance Imaging (MRI) technology, it emerged a new pathological feature of schizophrenia, i.e. alteration in myelin structure. As already mentioned, myelin exerts multiple functions and it is extremely responsive to environmental and cellular stimuli. Additionally, 
myelinated fibres connect and synchronise various brain regions, maybe also indirectly influencing neurotransmitter release. Thus, one could speculate that myelin alteration could be one of the schizophrenia sub-phenotype that might explain and link the misbalances of the other signalling pathways (Takahashi, Sakurai et al. 2011).

\subsubsection{Myelin abnormalities in schizophrenia patients}

It has been known for long time that connectivity and white matter are altered in schizophrenia (McGuire and Frith 1996, Zalesky, Fornito et al. 2011). Considering the already clarified importance of myelin in high cognitive functions and electrical synchrony among different brain regions, this is not surprising. The prolonged development of myelination in humans and the high adaptiveness of the white matter could indeed represent a double-edged sword. It would ensure the complexity of human reasoning, but it would also add a weakness to the CNS, since myelin is so highly responsive also to adverse stimuli (Miller, Duka et al. 2012, Haroutunian, Katsel et al. 2014).

It is not clear whether the abnormalities in myelin are causative of schizophrenia or just a consequence of the disease, yet numerous evidences confirm abnormalities in white matter tract in schizophrenia patients versus healthy controls and it is crucial to understand the biological and phenotypical meaning of these alterations.

Imaging studies pinpointed alteration in myelin ultrastructure and fibres bundles. In particular, several experimental evidences report a reduction in Fractional Anisotropy (FA) and in Magnetisation Transfer Ratio (MTR), mostly in the corpus callosum, in the cortex, in the arcuate fasciculus and in the uncinate fasciculus in schizophrenia patients (Kubicki, Park et al. 2005, McIntosh, Munoz Maniega et al. 2008, Sussmann, Lymer et al. 2009, Holleran, Ahmed et al. 2014, Li, Kale Edmiston et al. 2014). Abnormalities in white matter of the arcuate fasciculus are also associated with neurophysiological alteration in the N1 component suppression in schizophrenia patients (Whitford, Mathalon et al. 2011) and they are considered to be partially involved in the manifestation of auditory hallucination typical of schizophrenia patients (Hubl, Koenig et al. 2004, Whitford, Ford et al. 2012). This indicates that myelin alteration might be involved in the impairment of the electrical communication among different brain regions. Moreover, the white matter size is 
reduced in schizophrenia patients and it seems also to be affected by a faster ageing process (Kochunov, Glahn et al. 2013, Balevich, Haznedar et al. 2015).

Ultrastructural analysis of postmortem brain via conventional electron microscopy (EM) in the prefrontal cortex reveals a reduction of oligodendrocyte density and apoptotic/necrotic oligodendrocytes surrounded by microglia in patients versus healthy controls (Uranova, Orlovskaya et al. 2001, Uranova, Vostrikov et al. 2004, Vostrikov and Uranova 2011).

Proteomic and gene-expression studies further support imaging and EM findings, showing alteration of the expression of myelin-related genes and proteins in the prefrontal, frontal and anterior cingulate cortices(Table A - Appendix)(Hakak, Walker et al. 2001, Flynn, Lang et al. 2003, Tkachev, Mimmack et al. 2003). The altered expression of myelin gene further supports the involvement of myelin abnormalities in schizophrenia.

Among the several candidate gene/protein, in the last few years MBP has become more and more appealing for the attempt of understanding the role of myelin abnormalities in schizophrenia. In fact different laboratory showed a consistent reduction in MBP in patients with schizophrenia and other severe psychiatric illness versus control (Table 1). As a further support of the relevance of MBP reduction in schizophrenia, a recent convergent functional genomic study has reported MBP as one of the top genes involved in schizophrenia (Ayalew, Le-Niculescu et al. 2012). To elucidate the meaning and the consequences of myelin abnormalities (specifically MBP reduction) in schizophrenia, we need a model, that (1) presents abnormalities without major motor impairments, which would prevent the detection of subtle psychiatric phenotypes, and (2) shows a reasonable approximation of construct validity (Nestler and Hyman 2010).

In the attempt of understanding the "myelin hypothesis" of schizophrenia, a few mouse model of dysmyelination have been proposed (Table 2). However in some of them the dysmyelination was quite severe, for instance in Quaking mice hoistad (Hoistad, Segal et al. 2009), which does not resemble the condition in schizophrenia. In other models, demyelinationwas induced by toxic agent and, consequently, the damage to the fibres was quite unspecific, i.e. cuprizone model xiao makinodan (Makinodan, Yamauchi et al. 2009). Last but not least, none of this model specifically addressed the reduction of MBP. Thus, to fill this gap in the field of 
schizophrenia research, we decided to explore the biological and behavioural significance of a reduction in MBP. 
Table 1. Reduction of MBP in severe psychiatric illness (legend in the following page)

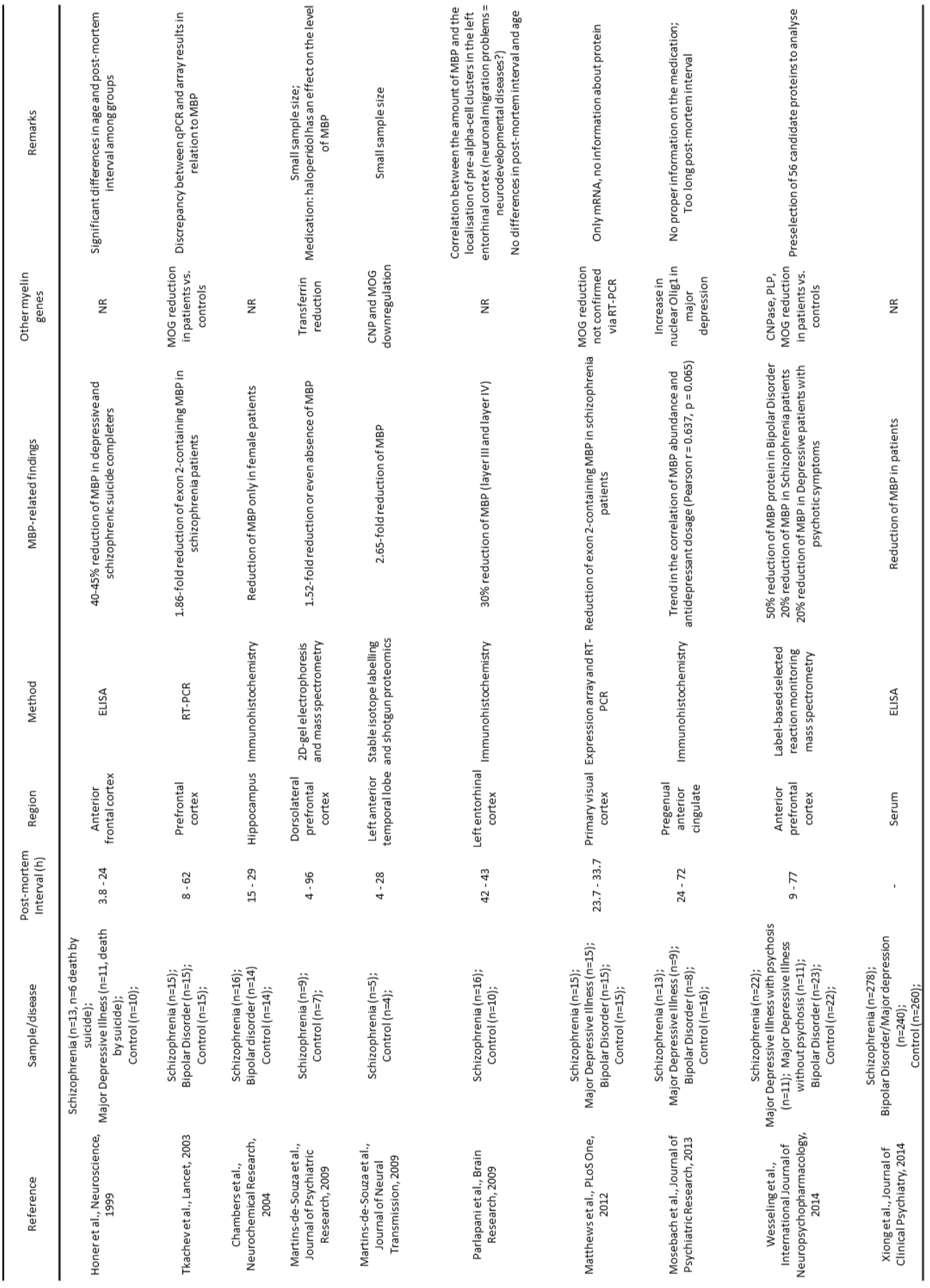


(previous page)Table 1 Reduction of MBP in severe psychiatric illness.

Reference from top to bottom: (Honer, Falkai et al. 1999, Tkachev, Mimmack et al. 2003, Chambers and Perrone-Bizzozero 2004, Martins-de-Souza, Gattaz et al. 2009, Parlapani, Schmitt et al. 2009, Matthews, Eastwood et al. 2012, Mosebach, Keilhoff et al. 2013, Xiong, Zeng et al. 2014, Wesseling, Gottschalk et al. 2015)

Table 2. Available animal models to study myelin alteration in schizophrenia

\begin{tabular}{|c|c|c|c|c|}
\hline Animal name & $\begin{array}{l}\text { Molecular findings related } \\
\text { to oligodendrocytes/myelin }\end{array}$ & Molecular findings (others) & Behavioral findings & Reference \\
\hline Bace1 knock out & Hypomyelination & $\begin{array}{l}\text { Reduced spine density in } \\
\text { hippocampal pyramidal neurons }\end{array}$ & $\begin{array}{l}\text { Deficits in prepulse inhibition, } \\
\text { cognitive function, and social } \\
\text { recognition, novelty-induced } \\
\text { hyperactivity, hypersensitivity } \\
\text { to MK-801 }\end{array}$ & $\begin{array}{l}\text { Hu et al. (2006), } \\
\text { Savonenko et al. (2008) }\end{array}$ \\
\hline ERBB4 heterozygotes & & & Hyperactivity & Stefansson et al. (2002) \\
\hline DN-ERBB4 & $\begin{array}{l}\text { Impaired oligodendrocyte/ } \\
\text { myelin development, } \\
\text { reduced conductance } \\
\text { velocity }\end{array}$ & Elevated dopamine signaling & $\begin{array}{l}\text { Increased sensitization to } \\
\text { amphetamine }\end{array}$ & Roy et al. (2007) \\
\hline MAG knock out & Subtle dysmyelination & Alterations in basal dendritic integrity & & \\
\hline $\begin{array}{l}\text { NgR1 (Nogo receptor) } \\
\text { knock out }\end{array}$ & & $\begin{array}{l}\text { Enhancement of long-term potentiation } \\
\text { and attenuates long-term depression }\end{array}$ & $\begin{array}{l}\text { Deficits in working memory, } \\
\text { reduced locomotor activity }\end{array}$ & Hsu et al. (2007) \\
\hline NRG1 heterozygotes & Controversial & $\begin{array}{l}\text { Reduced phosphorylation of NR2B, } \\
\text { impaired LTP }\end{array}$ & $\begin{array}{l}\text { Hyperactivity, reduced } \\
\text { prepulse inhibition }\end{array}$ & $\begin{array}{l}\text { Taveggia et al. (2008) } \\
\text { Stefansson et al. (2002) }\end{array}$ \\
\hline PLP1 transgenics & $\begin{array}{l}\text { Reduced conductance } \\
\text { velocity }\end{array}$ & & $\begin{array}{l}\text { Reduced prepulse inhibitions, } \\
\text { spatial learning and working } \\
\text { memory deficit. }\end{array}$ & Tanaka et al. (2009) \\
\hline PTPRZ1 transgenics & $\begin{array}{l}\text { Delayed oligodendrocyte } \\
\text { development }\end{array}$ & $\begin{array}{l}\text { Reduced phosphorylation of NR2B, } \\
\text { impaired LTP, elevated dopamine } \\
\text { signaling }\end{array}$ & $\begin{array}{l}\text { Reduced prepulse inhibitions, } \\
\text { spatial learning, hyperactivity, } \\
\text { increased sensitization to } \\
\text { amphetamine }\end{array}$ & $\begin{array}{l}\text { Takahashi et al. } \\
\text { (in preparation) }\end{array}$ \\
\hline Quaking & Severe dysmyelination & $\begin{array}{l}\text { Shorter dendritic lengths distal from the } \\
\text { soma, fewer numbers of branch radial } \\
\text { intersections, fewer higher order branches } \\
\text { were observed in apical dendrites } \\
\text { Increased dopamine metabolism } \\
\text { and dopamine D2 receptor binding, }\end{array}$ & & Hoistad et al. (2009) \\
\hline $\begin{array}{l}\text { Cuprizone } \\
\text { treated mice }\end{array}$ & Demyelination & Elevated dopamine signaling & $\begin{array}{l}\text { Deficits in working memory, } \\
\text { prepulse inhibition, and } \\
\text { social interaction }\end{array}$ & $\begin{array}{l}\text { Xiao et al. (2008), } \\
\text { Makinodan et al. (2009) }\end{array}$ \\
\hline
\end{tabular}

Table from (Takahashi, Sakurai et al. 2011)

References from top to bottom:(Stefansson, Sigurdsson et al. 2002, Hu, Hicks et al. 2006, Hsu, Woodroffe et al. 2007, Roy, Murtie et al. 2007, Savonenko, Melnikova et al. 2008, Taveggia, Thaker et al. 2008) tanaka (Tanaka, Ma et al. 2009)

\subsection{Shiverer heterozygous: a model to unravel schizophrenia sub-phenotype?}

Ablation of MBP can be achieved by deleting 5 of the 7 exons of the allele coding for the classical MBP (see section 2.2.4). When the deletion interests both MBP alleles, as in shiverer mice, this natural occurring mutation leads to complete loss of myelin compaction in the CNS. Consequently these mice show a strong motor phenotype (they are literally shivering) in parallel to a very limited lifeexpectancy (Chernoff 1981, Readhead, Takasashi et al. 1990).

Due to the motor impairment and to the short life expectancy, this model does not fulfil our translational purposes of understanding mild dysmyelination in schizophrenia. On 
the contrary, heterozygous shiverer (here referred to as $\mathrm{Mbp}+/$ - mice) miss only one allele coding for the classical MBP. As consequence, these mice express 50\% MBP mRNA and protein, but this amount has been claimed to be sufficient to ensure normal motor performances on the rota-rod and normal myelination (Barbarese, Nielson et al. 1983, Kuhn, Petroulakis et al. 1995). Indeed, myelination of the optic nerve of Mbp+/wasreported to be completely comparable to the one in the wild-type (Shine, Readhead et al. 1992); moreover, the phenotype of the shiverer can be rescued with $25 \%$ of the physiological amount of MBP, supporting the idea the one $M b p$ allele could be more than sufficient to ensure myelination and myelin compaction (Readhead, Popko et al. 1987).

Due to the claimed comparability between $\mathrm{Mbp}+/$ - and wild-type mice, $\mathrm{Mbp}+/-$ mice have been neglected or even used as "healthy controls"(Tu, Kim et al. 2013).

However, it recently emerged that this mouse model might have been overlooked. Visual Evoked Potential (VEP) measurements comparing $\mathrm{Mbp}+/-$ mice and wildtype revealed a mild but significant increase $(+7 \%)$ in the latency of the neurophysiological component. This readout might disclose a mild impairment in brain electrical synchrony (Martin, Hiltner et al. 2006), phenotype often found in schizophrenia patients. Furthermore, a developmental delay in myelin compaction and an alteration in the brain metabolism of young $\mathrm{Mbp}+/$ - mice have been reported (Snaidero, Mobius et al. 2014, Takanashi, Nitta et al. 2014). This could underscore a more general delay in developmental milestones, often reported in schizophrenia patients before the onset of the pathology (Welham, Isohanni et al. 2009).

These subtle anomalies that emerged in $\mathrm{Mbp}+/$ - mice suggest that a mild reduction in MBP might not be detrimental for gross motor functions and basic behaviour, but it could have an effect on the fine tuning of the brain. Indeed schizophrenia is not characterised by extreme dysmyelination and motor deficits, but by a subtle alteration in myelin structure, accompanied by misbalances of neurotransmitter and metabolites and by neural desynchronisation.

For these reasons, Mbp+/- mouse appeared to be a suitable model to explore the consequences of a suboptimal MBP amount, often detected in schizophrenia. Since it is not clear at which time in life the reduction of MBP has a pathological effect on the patients, we screened this mouse model at different time points, i.e. young (3 months), mature (6 months) and senior (>17 months). This allowed us to estimate (1) 
the impact of such reduction on myelin structure in a life-span fashion and from there (2) to explore more in depth the behavioural and biological consequence of a "mild demyelination sub-phenotype", with the final aim of unravelling at least part of the complex disease called schizophrenia 


\section{METHODS AND MATERIALS}

All experiments were approved by the local animal care and use committee in accordance with the German animal protection law. Mice were group-housed (4-5 animals) in standard plastic cages and they had access to food and water ad libitum. They were kept under a 12h light-dark cycle (lights on at 7:00am) and an ambient temperature of $20-22^{\circ} \mathrm{C}$.

\subsection{RNA extraction}

Three-month, 6-month and 20-month old animals were anaesthetised with $1 \mathrm{~mL}$ of avertin ( $1 \mathrm{~g}$ tribromomethanol, $1 \mathrm{~mL}$ o tetramylalchol in $71,49 \mathrm{~mL}$ milliQ water) and the prefrontal cortex and brainstem were dissected from(4 animals per genotype) and stored at $-80^{\circ} \mathrm{C}$ before analysis. The tissue was homogenised in Qiazol via mechanical breakage, chloroform was added to the homogenised suspension and the samples were centrifuged at $12000 \mathrm{~g}$ for $15 \mathrm{~min}$ at $4{ }^{\circ} \mathrm{C}$ to induce separation of the RNA from DNA and protein content. The RNA extraction was performed via miRNeasy mini kit (QIAGEN \#217004) following the manufacture's instruction. The RNA concentration was measured via detection of the sample absorbance at OD260nm and the RNA purity was evaluated via OD260/OD280. The samples were stored at $-80^{\circ} \mathrm{C}$.

\subsection{Retrotranscription and Real-time PCR}

To measure the mRNA level of MBP, CNP, PLP, each RNA sample was diluted to 20 ng/ $\mu \mathrm{L}$ mixed with $0.6 \mathrm{pmol}$ oligo dT-mix, $120 \mathrm{pmol}$ of Random Hexamer (Roche) and retrotranscribed to cDNA with SuperScript ${ }^{\circledR}$ III Reverse Transcriptase kit (LifeTechnologies) according to the manufacturer's guideline.

The derived cDNA was then diluted 1:5 and $4 \mu \mathrm{L}$ of it were used as template for SYBR GREEN-based real-time PCR, accordingly to manufacturer's protocol. The reaction was run in Roche LightCycler 480, each sample was assayed in triplicate and normalised to 18 s ribosomal subunit. The following primers were used to amplify the construct of interest:

MBP Frw 5'-ACGGACACCCTTCCAAGTT-3' 
MBP Rev 5'-GTGTGCCTCACCGTGAAAA-3'

CNP Frw 5'-TAACCCTCCCTTAGCCCCTG-3'

CNP Rev: 5'-GTCCCTAGCATGTGGCAGCT-3',

PLP Frw 5'-GGCTAGGACATCCCGACAAG-3'

PLP Rev 5'-GCAAACACCAGGAGCCATACA-3', 18s Frw 5'-GCTCTAGAATTACCACAGTTATCCAA-3'

18s Rev 5'-AAATCAGTTATGGTTCCTTTGGTC-3'.

\subsection{Protein extraction and western blotting}

Three-month, 6-month and 20-month old animals were anaesthetised with $1 \mathrm{~mL}$ of avertin ( $1 \mathrm{~g}$ tribromomethanol, $1 \mathrm{~mL}$ o tetramylalchol in $71,49 \mathrm{~mL}$ milliQ water). The prefrontal cortex and brainstem were dissected and stored at $-80^{\circ} \mathrm{C}$ before analysis. The tissue was homogenised in lysis buffer $(50 \mathrm{mM}$ Tris/Cl pH 7.5, $150 \mathrm{mM} \mathrm{NaCl}, 1 \mathrm{mM}$ EDTA, $1 \%$ Triton $\mathrm{x}-100,0.5 \%$ Sodiumdeoxycholat) with the addition of freshly prepared protease inhibitors $(50 \mu \mathrm{g} / \mathrm{mL}$ Leupeptin, $0.2 \mathrm{mM} \mathrm{PMSF}, 1 \mathrm{mM}$ of activated sodium orthovandate, $30 \mu \mathrm{g} / \mathrm{mL}$ Aprotinin), incubated on ice for 30 minutes and centrifuged at $9000 \mathrm{rpm}$ at $4^{\circ} \mathrm{C}$ for 10 minutes. Part of the samples was used to assess protein concentration via Folin phenol reagent (Lowry, Rosebrough et al. 1951). And the rest was mixed with $4 \mathrm{X}$ Lämmli buffer and incubated at room temperature for 30 minutes. Comparable amounts of total protein from each experimental condition were separated on $15 \%$ SDS polyacrylamide gels and transferred onto nitrocellulose.

The membranes were blocked for at least $1 \mathrm{~h}$ at room temperature (Tris-buffered saline, $1 \%$ Tween, $5 \%$ powder milk) and incubated overnight at $4{ }^{\circ} \mathrm{C}$ with primary antibody: anti-MBP (1:2000, Dako), anti-CNP (1:5000, Sigma-Aldrich), anti-PLP (homemade, A431, 1:500) and anti- $\alpha$-Tubulin (1:10 000, Sigma-Aldrich). The membranes were then incubated with secondary antibody, either anti-mouse or anti-rabbit (1:5000, Rockland) for $2 \mathrm{~h}$ at room temperature. The intensity of the bands was detected via Odyssey Infrared Imaging system and quantified via Image Studio Lite software. The intensity of MBP, CNP and PLP were normalised to the intensity of $\alpha$-Tubulin.

\subsection{MRI (in collaboration with Prof. Boretius, who provided the method part)}

The mice were initially anesthetized with $5 \%$ isoflurane, subsequently intubated and kept under anaesthesia with $1.75 \%$ isoflurane in ambient air and 5\% oxygen by active 
ventilation with a constant respiratory frequency of 85 breaths per minute (Animal Respirator Advanced ${ }^{\mathrm{TM}}$, TSE Systems, Germany).

Magnetic resonance imaging (MRI) and magnetic resonance spectroscopy (MRS) were performed at a magnetic field strength of 7T (ClinScan, Bruker BioSpin, Germany). MRI comprised T2-weighted images (2D FSE, TR/TE=4000/50 ms), diffusionweighted images (2D EPI, TR/TE $=7000 / 28 \mathrm{~ms}, 12$ directions, $b=0 / 1000 \mathrm{~s} / \mathrm{mm}^{2}$ ) and 3 differently weighted 3D FLASH based datasets $\left(\mathrm{TR} / \mathrm{TE}=28 / 1.9 \mathrm{~ms}\right.$, flip angle $=25^{\circ}$ for $\mathrm{T} 1$ weighting and $5^{\circ}$ for proton weighting, the later with and without additional magnetization-transfer weighting by Gaussian-shaped off-resonance pulses with a flip angle of $500^{\circ}$ and an off-resonance frequency of $1200 \mathrm{~Hz}$ ). Localized proton MR spectra (PRESS, TR/TE $=6000 / 10 \mathrm{~ms}$ ) were obtained from a volume of interest (VOI) in the hippocampus $\left(1.8 \times 0.7 \times 1.8 \mathrm{~mm}^{3}\right)$, cortex $\left(3.9 \times 0.7 \times 3.2 \mathrm{~mm}^{3}\right)$ and corpus callosum $\left(3.9 \times 0.7 \times 1.7 \mathrm{~mm}^{3}\right)$. Metabolite quantification involved spectral evaluation by LCModel (Version 6.3-0G, Provencher, 1993). Results with Cramer-Rao lower bounds above $20 \%$ were excluded from further analyses.

Maps of magnetization transfer ratio (MTR), magnetization saturation (MT sat), fractional anisotropy (FA), apparent diffusion coefficient (ADC), axial diffusivity (AD), and radial diffusivity (RD) were calculated using in-house Matlab scripts (Mathworks, Natick, USA).

\subsection{Electron Microscopy (performed in collaboration with Dr. Möbius)}

Each animal $(\mathrm{Mbp}+/-=3$; WT = 3) was anaesthetised with avertin and intracardially perfused with $15 \mathrm{ml}$ of HBSS, followed by $50 \mathrm{ml}$ of fixative $(2.5 \%$ glutaraldehyde, $4 \%$ paraformaldehyde in PBS). The brains were dissected and $200 \mu \mathrm{m}$ coronal sections were cut by using a Leica VT1200S Vibratom (Leica Microsystems, Wetzlar). The genu of the corpus callosum was punched out of the sliced tissue and it was post-fixed overnight and embedded in Epon (Serva) after postfixation with $2 \% \mathrm{OsO}_{4}($ Science Services, Munich, Germany) and dehydration with ethanol and propylenoxid. Semi-thin sections were stained with methylene blue and AzurII. Ultrathin sections were contrasted with $1 \%$ uranyl acetate and lead citrate. Ultra-thin sections were scanned via Zeiss EM900 Elektron-Microscop (Zeiss, Oberkochen, Germany) and digital pictures were obtained using the wide-angle dual speed 2K-CCD-Camera (TRS, Moorenweis, Germany). 
Myelin and axon tracings were performed on the electron microscopy pictures (at least 15 pictures per animal) via ImageJ (http://rsb.info.nih.gov/ij/). g-ratio, as myelin thickness index, and t-ratio, as inner tongue ratio, were calculated as indicated in figure $14 \mathrm{~F}^{\prime}$.

\subsection{Tissue fixation and immunohistochemistry}

Fourteen-month old animals were anaesthetised with $1 \mathrm{~mL}$ of avertin (1g tribromomethanol, $1 \mathrm{~mL}$ o tetramylalchol in $71,49 \mathrm{~mL}$ milliQ water) and perfused with $4 \%$ formaldehyde (FA). The fixes brains were dehydrated in $30 \%$ sucrose solution and stored at $-80^{\circ} \mathrm{C}$. Coronal sections $(30 \mu \mathrm{m}$-thick) were stained with microglia marker antiIba1 (Wako, 1:1000 in 5\% normal horse serum and 0.5\% TritonX-100 in 1x PBS), followed by anti-rabbit (BRAND, donkey anti-rabbit, 594nm). Cell nuclei were counterstained with DAPI (4',6-Diamidino-2-phenylindole 1:10000, Brand).

Eighteen-month old animals were anaesthetised with $1 \mathrm{~mL}$ of avertin $(1 \mathrm{~g}$ tribromomethanol, $1 \mathrm{~mL}$ o tetramylalchol in $71,49 \mathrm{~mL}$ milliQ water) and perfused with $4 \%$ formaldehyde (FA). The fixes brains were dehydrated in $30 \%$ sucrose solution and stored at $-80^{\circ} \mathrm{C}$. Coronal sections $(30 \mu \mathrm{m}$-thick) were stained with myelin markers antiMBP (Sternberger, 1:800 in 3\% horse serum and 0.5\% TritonX-100 in 1x PBS), followed by anti-mouse (AF555, goat anti-mouse), and with anti-PLP (homemade, A431, 1:500 in 3\% horse serum and 0.5\% TritonX-100 in 1x PBS), followed by antirabbit (AF488, goat anti-rabbit).

The brain slices were dried, mounted with Mounting Medium and scanned with inverted epifluorescence microscope (LEICA, DMI 6000B). The analyses were performed via IMARIS software (http://www.bitplane.com/) and Fiji (http://fiji.sc/Fiji).

\subsection{Behavioural screening}

The behavioural screening of adult (>3-months old) and old (>17-months old) male mice was performed on 1-4 cohorts (detailed information in Table 1) of at least 10 animals per genotype (MBP+/- vs. wild-type littermate). All the experiments were conducted by investigators blind to the genotype, during the light phase of the day (9:00-17:00). Both basic and high behavioural functions were investigated. 


\subsubsection{Elevated plus maze}

The plus-shape apparatus consisted of two walled and two open arms elevated from the ground. The light intensity during the test was set to 130lux. The mouse was placed in the central platform, facing the edge where an open arm encountered a walled arm. Behaviour was recorded over 5 min by an overhead video camera. A personal computer equipped with Viewer software (Biobserve, Bonn, Germany) measured the locomotion (time and speed) of the animal in the open and walled arms respectively. The proportion of time spent in open arms (natural aversion) was used as an anxiety equivalent.

\subsubsection{Open field}

The apparatus consisted of a $110 \mathrm{~cm}$-diameter walled arena. The light intensity during the test was set to 130 lux. The mouse was released in the centre of the arena and the latency to reach the periphery was recorded (cutoff: 180'). After reaching the periphery (or after 180"), the mouse was allowed to freely move in the arena for 7 minutes. A personal computer equipped with Viewer software (Biobserve, Bonn, Germany) measured the locomotion (time, velocity and total distance) of the animal in the periphery, intermediate, centre of the arena. The time spent in the periphery (thigmotaxis) was used as an anxiety equivalent and the locomotion (average velocity and total distance) was referred as motor performances.

\subsubsection{Hole-board (1 day)}

The apparatus consisted of a chamber $(21 \times 21 \times 36 \mathrm{~cm})$ with a floor raised $5 \mathrm{~cm}$ above the bottom of the chamber with 16 equally spaced holes $(2 \mathrm{~cm}$ in diameter). The animal was allowed to freely explore the apparatus for 5 minutes and the number of nose-pokes in the hole of the floor was recorded via an infrared photo beams connected to a computer with ActiMot software version 2 (TSE Systems GmbH, Bad Homburg, Germany).

\subsubsection{Pre-pulse inhibition of startle response (PPI)}

The animals were allocated in metal cages $(82 \times 40 \times 40 \mathrm{~mm})$ and placed on a transducer platform that recorded movements inside an attenuating cabinet (TSE Systems, Bad Homburg, Germany). Startle reflex was induced by acoustic stimuli emitted by a loudspeaker inside the cabinet. The startle response to the acoustic stimulus was 
recorded for $260 \mathrm{~ms}$ from the onset of the pulse. The animal underwent $2 \mathrm{~min}$ habituation to a $65 \mathrm{~dB}$ background white noise (constant over the whole experimental phase) and the reaction to it was recorded over 1 minute. Then, $6120 \mathrm{~dB}$ stimuli of $40 \mathrm{~ms}$ duration were applied to decrease the influence of within-session habituation. To test PPI, the startle pulse (120dB intensity) was applied either alone or proceeded by a $20 \mathrm{~ms}$-pre-pulse stimulus of 70/75/80dB. A delay of $100 \mathrm{~ms}$ with background noise was interposed between the presentation of the pre-pulse and pulse stimulus. The trials were pseudo-randomized with a variable interval ranging from 8-22 second. The amplitude of the startle response (expressed in arbitrary units) was defined as the difference between the maximum force detected during the recording window and the force measured immediately before the stimulus onset. For each animal, the amplitudes were averaged separately for the startle reflex to the pulse (startle response), or for the startle reflex to the pulse preceded by a pre-pulse, or for the startle reflex without any acoustic stimulus. PPI was calculated as the percentage of the startle response using the following formula: $\%$ PPI=100-[(startle amplitude after pre-pulse)/(startle amplitude after pulse only) $\times 100]$.

\subsubsection{Acoustic Startle Response}

To assess the sensorimotor reflex the animals were allocated in metal cages $(82 \times 40 \times 40 \mathrm{~mm})$ and placed on a transducer platform that recorded movements inside an attenuating cabinet (TSE Systems, Bad Homburg, Germany). Startle reflex was induced by acoustic stimuli emitted by a loudspeaker inside the cabinet and it was measured over a period of $100 \mathrm{~ms}$. The habituation phase consisted in the administration of background noise $(65 \mathrm{~dB})$ for $2 \mathrm{~min}$ and it was followed by a recording of the baseline startle of the each animal at $65 \mathrm{~dB}$ for $1 \mathrm{~min}$. The experimental phase consisted in the pseudo-randomised presentation of 40ms-duration acoustic stimuli ranging from a minimum of $65 \mathrm{~dB}$ to a maximum of $120 \mathrm{~dB}$ (inter-stimulus interval 8-22s). Each stimulus was administer 10 times. The average startle response amplitude to each stimulus was used for statistical analysis.

\subsubsection{Rota-rod}

The apparatus comprises a rotating drum (Med Associates Inc. Georgia, Vermont) that is accelerated from 4 to 40 revolutions per minute over the course of 5 minutes. The animal was placed on the drum and the latency to fall from the drum was detected via a 
laser sensor located on the floor of the apparatus and recorded by the instrument. When the assessment of motor learning was intended, the test was repeated $24 \mathrm{~h}$ after the first session.

\subsubsection{Visual cliff}

The apparatus consisted of a transparent rectangular plastic surface, half of it located on a grey plastic supporter (ground zone) and the other half standing over the edge of the supporter, 1 meter above the floor (air zone). The animal was released on the edge of the grey plastic supporter and it was allowed to explore the area for 5 minutes. The activity was recorded via an overhead camera connected to the video-tracking system Viewer 2 (Bioserve, $\mathrm{GmbH}$ ). The percentage of time spent by the animal in the air zone was consider as index of visual acuity (the less time the better the vision acuity).

\subsubsection{Grip strength}

The animal was lifted by the tail and it was placed on a grip strength meter (TSE System, GmbH), so that the forepaws could grasp a wire grid and the body could be parallel to the table surface. The animal was then gently pulled by its tail until it releases the wire. The force applied by the mouse to hold on to the wire was recorded in ponds. At least 3 trials per animal were carried out and the average of the values recorded was used for statistical analysis.

\subsubsection{Hot plate}

The animal was placed on a metal plate (Ugo Basile Srl, Comerio, Italy) that was preheated up to $55^{\circ} \mathrm{C}$. The latency to show hind paw licking or jumping was recorded (cut off 60s). The animal was then immediately removed from the plate, to prevent injuries.

\subsubsection{Buried food test}

The first part of the test (day 1 to day 6) aimed to habituate the animal to the test environment $(29.5 \times 18.5 \times 13 \mathrm{~cm}$ cages) and to the test food (crunchy chocolate cookies). On the first 3 days the animal was placed in the test cage 2 x 20 minutes per day. At the end of day 3, the regular food pellets in the home cage of the test animal were replaced by test food. From day 4 to day 6 the animal was placed in the test cage 
containing the testing food ( 2 x 20 minutes). On day 7 the animal was placed closed to the wall of the testing cage, where the testing food was hidden under $1.5 \mathrm{~cm}$ standard bedding at the opposite side of the cage. The time from the moment the mouse was released into the cage to the time it located the cookie and initiated burrowing was recorded (cut off 300 seconds). To control for other sensory, motor, or motivational deficiencies that might influence the food-finding latency, the test was repeated, with the test food placed on the bedding (visible to the mouse). All the steps performed in the test cage were carried out at light intensity of 50-60lux.

\subsubsection{Marble burying}

The light intensity was set to 60lux. A large test cage $(29.5 \times 18.5 \times 13 \mathrm{~cm})$ was filled with $5 \mathrm{~cm}$ deep layer of bedding and 24 marbles were arranged in a $6 \times 4$ array. The animal was released in the centre of the cage and let freely explore for 30 minutes. The number of marbles buried was counted. Marbles are considered buried if at least twothirds of the marble is obscured from view by bedding.

\subsubsection{Y-maze}

The apparatus consisted of a three walled-arms radiating maze with triangle-shaped central platform. The animal was released in the central area, facing the edge where two arms encountered, and it was allowed to freely explore the maze for $5 \mathrm{~min}$. Total number of entries, number of triplets (the number of consecutive choices of each of the three arms, without re-entries during the last three choices), and alternation-ratio (number of triplets/(number of entries - 2)) were recorded. An arm entry was scored when the mouse entered an arm with all four paws.

\subsubsection{Three-chambered social test}

The test was adapted from previously described methods(Moy, Nadler et al. 2004). The social testing arena consisted of a rectangular, 3-chambered box. Transparent Plexiglas walls, with rectangular openings, separated the three compartments of the arena. The experimental procedure consisted of 4 phases: habituation, sociability, control for place preference biases and social memory. During habituation phase, the test mouse was first placed in the middle compartment and the 5-minute exploration was restricted to this area. During sociability phase, a new C57BL/6N male mouse of the same age 
(stranger 1) was located inside a rectangular wire-mesh cage in one of the side chamber. An identical empty wire cage was placed in the opposite chamber. The test animal was then allowed to access all the three compartments of the arena for 10 minutes. During control for place preferences biases phase Stranger 1 was then moved to the other side compartment and the test animal was allowed to explore the arena for other 10 minutes. In social memory phase a second stranger (stranger 2) was placed in the previously empty wire-mesh cage. The test mouse was allowed to freely explore the arena, having now the choice between the stranger 1 (familiar) and a new stranger (novel). The time spent and the number of entryin each compartment was recorded by the video-tracking system Viewer 2 (BIOBSERVE GmbH). Sociability and social memory were calculated as follow:

$$
\begin{gathered}
\text { Sociability }=\left[\text { time }_{\text {stranger }} /\left(\text { time }_{\text {stranger }}+\text { time }_{\text {empty }}\right)\right] \text { x } 100 \\
\text { Social memory }=\left[\text { time }_{\text {novel }} /\left(\text { time }_{\text {novel }}+\text { time }_{\text {familiar }}\right)\right] \text { x } 100
\end{gathered}
$$

\subsubsection{Sucrose preference}

The animal was first habituated for 48 hours to consume $2 \%$ sucrose solution from two $100 \mathrm{~mL}$ bottles. After the habituation session, the animal was water-deprived for 3 days. On each day of water deprivation, the animal was in contact for 60 minutes with two bottles (one with tap water and one with $2 \%$ sucrose solution) in the home cage. Consumption of water or sucrose solution was measured by weighing the bottles before and after the session. The percentage of sucrose preference (percentage) was calculated as follows:

Sucrose preference $=[($ sucrose solution intake/total liquid intake $)] \times 100$

\subsubsection{Morris water maze}

The apparatus consisted of a $110 \mathrm{~cm}$-diameter tank filled with opaque water $\left(25 \pm 1^{\circ} \mathrm{C}\right.$, depth $300 \mathrm{~cm})$ and of a submerged escape-platform $(10 \mathrm{~cm} \times 10 \mathrm{~cm}, 1 \mathrm{~cm}$ below water surface). The animal swim pattern was documented by a video-tracking system (Viewer2, Biobserve GmbH, Germany). Escape latency, swim speed, and path length were recorded. The light intensity was set to $\sim 1001$ ux.

The test consisted 5 main phases (17 day in total): visible platform ( 2 days), acquisition (hidden platform, 8 days), probe trial (1 day), reverse learning (hidden platform, 4 days), reverse probe trial (1 day). 
Visible platform: the animal was trained for 2 days (4-trials/day) to find a submerged platform positioned in the centre of one of the four quadrants of the pool. A visual hint was given to the mouse by a small flag fixed on the surface of the platform.

Acquisition (hidden platform): the animal was trained for 8 days (4-trials/day) to find a submerged platform positioned in the centre of one of the four quadrants of the pool. Extra-maze cues were placed on the walls of the testing room to help orientation. The animal was released into the water facing the pool wall at one of four start locations and allowed to search for the platform for a maximum of 90s. A mouse that failed to find the platform within 90s was guided to the platform and remained there for 20s before being removed from the pool.

Probe trial: on day 11, the platform was removed from the pool and the animal was allowed to freely swim for 90s. The percentage of time spent in each quadrant of the pool was recorded.

Reverse learning (hidden platform): The animal was trained for further 4 days (4trials/day) to find a submerged platform moved to the centre of a different quadrant of the pool.

Reverse probe trial: on day 17 on day 11, the platform was removed from the pool and the animal was allowed to freely swim for 90s. The percentage of time spent in each quadrant of the pool was recorded.

\subsubsection{Novel object recognition (without and with delay)}

The test was adapted from previous published method(Bevins and Besheer 2006). The apparatus consisted of a grey plastic squared-box where plastic object were located accordingly to the test procedure. The tests were applied to adult animals ( $>3$ months). They both comprised two phases: exploration phase and memory phase that were separated by a 30-minute time interval in case of novel object recognition with delay.

During the exploration phase two objects of the same colour and similar shape were located in two opposite zone of the test arena. The animal was then released in the box and it was allowed to freely explore for 10 minutes. During the memory phase one of the two familiar object was substituted by a new one (black and of a totally different shape). 


\subsubsection{Novel object recognition (2)}

The test was adapted from previous published method(Roullet, Mele et al. 1996). The apparatus consisted of an arena with grey plastic wall and a $10 \times 8 \mathrm{~cm}$ striped cue located in the inside surface. The following test was performed with old animals (>17 months) and it comprised 7 sessions of 7 minute each; in each session the animal was allowed to freely explore the environment and a video-tracking system (Viewer2, Biobserve GmbH, Germany) was recording the animal motion and exploration. In the first session, the arena was empty (habituation). From session 2 to session 4 , four object of the same colour (white) were located at the 4 poles of the apparatus (object exploration). In sessions 5 and 6 , one of the objects was moved to the centre of the arena. During session 7 (memory phase), one of the familiar white object was exchanged with a black one.

\subsubsection{LABORAS - spontaneous home cage behaviour}

The LABORAS apparatus consisted of a sensor platform (Carbon Fiber Plate $1000 \mathrm{~mm}$ $\times 700 \mathrm{~mm} \times 700 \mathrm{~mm} \times 30 \mathrm{~mm}$, Metris B.V., Hoofddorp, Netherlands), positioned on two force transducers and a third fixed point attached to a heavy bottom plate (Corian Plate $980 \mathrm{~mm} \times 695 \mathrm{~mm} \times 695 \mathrm{~mm} \times 48 \mathrm{~mm}$ ). The whole structure stood on three spikes able to absorb vibrations. The animal was located in transparent polycarbonate cages (Makrolon type II cage, $22 \mathrm{~cm} \times 16 \mathrm{~cm} \times 14 \mathrm{~cm}$ ) with a thin layer of bedding covering the floor. The cage (including the top-grid, food and drinking bottle) was suspended over the sensing platform. The animal was single-housed in the LABORAS cage from 17:00 until 9:00 on the following day and the mechanical vibration derived from the animal behaviour were converted to electrical signals by the force transducers, amplified and stored in a computer. LABORAS software was then used to process the data and to retrieve information about the following behavioural readouts: eating, drinking, scratching, circling, climbing, immobility, locomotion, and grooming.

\subsubsection{Grasping test}

The test was adapted from the combination of the reaching chamber test(Qian, Lei et al. 2010)and the paw preference test(Collins 1975, Ribeiro, Eales et al. 2013).

The apparatus consisted of four in-line white plastic chambers with an exchangeable transparent plastic panel on the front. On each panel, in correspondence of each chamber and evenly distanced from the left and right sides of the chamber, were located 
four feeding tubes. Three panels with three different tube diameters $(10 \mathrm{~mm}, 8 \mathrm{~mm}$ and $5 \mathrm{~mm}$ ) were used. When required by the protocol, a guillotine door was manually used to obstruct the feeding tubes.

Each animal underwent an experimental procedure three phases: (1) 2-day food habituation phase, when the experimental pellets were introduced in the home-cage of the animal; (2) 2-day learning phase, when the animal had to learn to retrieve the pellet from the feeding tube (day 1: big diameter, guillotine door always open; day 2: intermediate diameter, guillotine door closing every minute for 10 minutes); (3) 1-day testing phase, when the animal had to retrieve the pellet from the smallest diameter feeding tube (10 trials of 1 minute each). The following parameters were measured: paw preference, efficiency index (success in retrieving/attempts in retrieving), number of successes at the first attempt, time to approach the pellet, time to retrieve the pellet, grasping score $(1=\operatorname{good}$ grasp; $0=$ wrong grasp; $0.5=$ the animal used the paw to move the pellet closer to the snout, but then it used the tongue to retrieve it)

\subsection{Statistical Analysis}

Between-group comparisons were performed via t test for independent samples and two-way analysis of variance (ANOVA or repeated-measures ANOVA). Within-group chance level performance (ratio or percentage values) was carried out via single group t tests against a chance level. Mann-Whitney $\mathrm{U}$ and Wilcoxon tests were applied when normality assumption was violated (Kolmogorov-Smirnov test). All statistics were performed using Prism GraphPad software or MatLab scripts (Mathworks, Natick, USA). Data presented in the figures and text are expressed as mean \pm SEM; $p$ values $<0.05$ were considered significant. 


\section{RESULTS}

Several studies in post-mortem brains from schizophrenia patients revealed a reduction of MBP (mRNA and protein) up to 40\% (Table 1). To understand the consequence of such reduction we extensively characterised heterozygous shiverer, here called Mbp+/mice, in comparison with wild-type.

Specifically, we attempted to clarify whether the reduction of MBP expression was causative of:

(1) anomalies in the expression of other highly expressed myelin proteins, namely CNP and PLP

(2) fibres damage and subtle demyelination

(3) alteration of brain metabolism and neurotransmitters

(4) behavioural alteration, in particular in the context of complex functions (sociability, learning, memory, sensorimotor gating)

\subsection{MBP show an age-dependent and area-specific expression pattern}

The first step to validate the mouse model was to confirm the reduction in MBP (mRNA and protein). Since it is not clear when the changes in MBP expression happen and because of the documented ageing effect on myelin and myelin-related molecules (Hagemeyer, Goebbels et al. 2012, Young, Psachoulia et al. 2013, Haroutunian, Katsel et al. 2014), three different time points were selected for the analysis, i.e. 3 months (young animals), 6 months (mature animals) and 20 months (senior animals).

The quantification was restricted to two "contrasting" brain areas, i.e. prefrontal cortex (PFC) and brainstem. PFC is mainly composed of grey matter, it is one of the brain area highly affected in schizophrenia patients (Perlstein, Carter et al. 2001)and it is involved in higher cognitive/social functions in both human and mice (DeVito, Lykken et al. 2010, Miller, Duka et al. 2012). In contrast, brainstem is mainly composed of white matter and it is more devoted to basic function.

As expected, real-time PCR revealed that the percentage of $M b p$ mRNA was significantly reduced in $\mathrm{Mbp}+/$ - compared with wild-type mice in both $P F C$ and in brainstem at 3 and 6 months (PFC: 3 months $\mathrm{p}=0.007,6$ months $\mathrm{p}=0.02$, MannWhitney; brainstem: 3 months $\mathrm{p}=0.002,6$ months $\mathrm{p}=0.001$; Mann-Whitney; wild-type $\mathrm{n}=4$, Mbp+/- n=4; Fig. 7 A, B, E, F). At 20 months the gene dosage did not affect the 
expression level anymore. In fact, the percentage of $M b p$ mRNA was not strictly significantly reduced in $\mathrm{Mbp}+/-$ vs. wild-type $(P F C: \mathrm{p}=0.08$, Mann-Whitney; brainstem: $\mathrm{p}=0.1$, Mann-Whitney; wild-type $\mathrm{n}=4$, Mbp+/- $\mathrm{n}=4$ Fig. $7 \mathrm{C}, \mathrm{G})$. Independently of the genotype, $M b p$ expression tends to increase upon ageing in $P F C$ (Mbp: $\mathrm{p}=0.03$, two-way ANOVA; $\mathrm{p}=0.08$, Jonckheere Terpstra test; wild-type $\mathrm{n}=4$, $\mathrm{Mbp}+/-\mathrm{n}=4$ per time point;Fig. 7D), but not in the brainstem (Mbp: $\mathrm{p}=0.13$, two-way ANOVA; $p=0.52$, Jonckheere Terpstra test; wild-type $n=4, M b p+/-n=4$ per time point; Fig $7 \mathrm{H}$ ). This could be caused by a homeostatic response of the PFC to a general diminishment of MBP protein level upon ageing.

3 mo
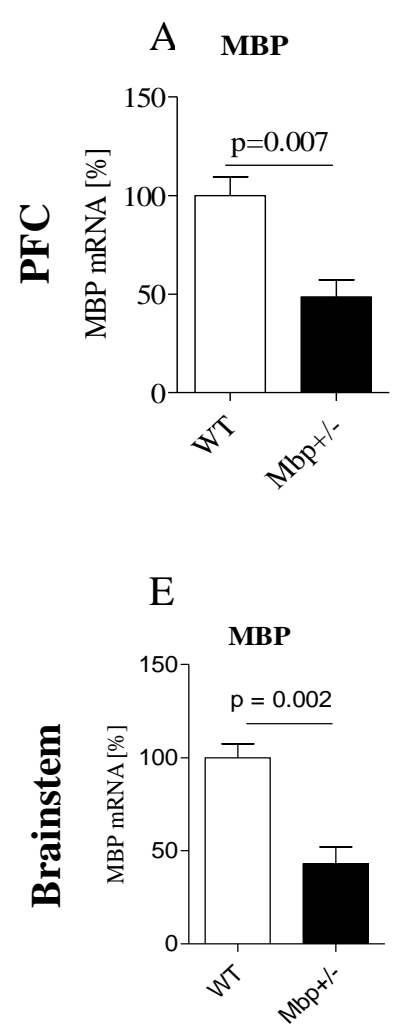

6 mo
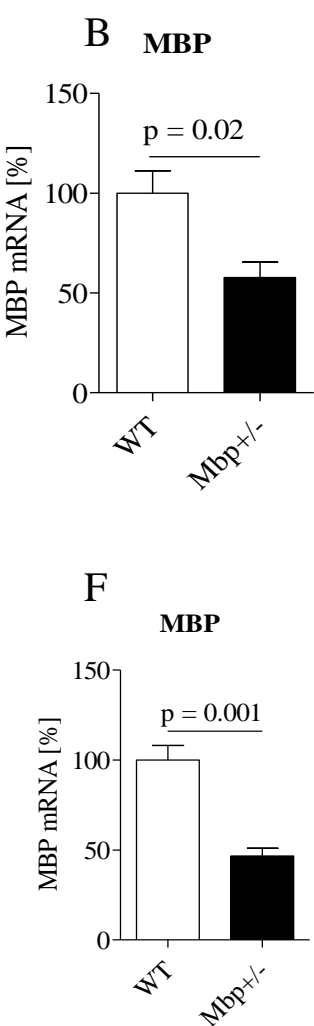

20 mo
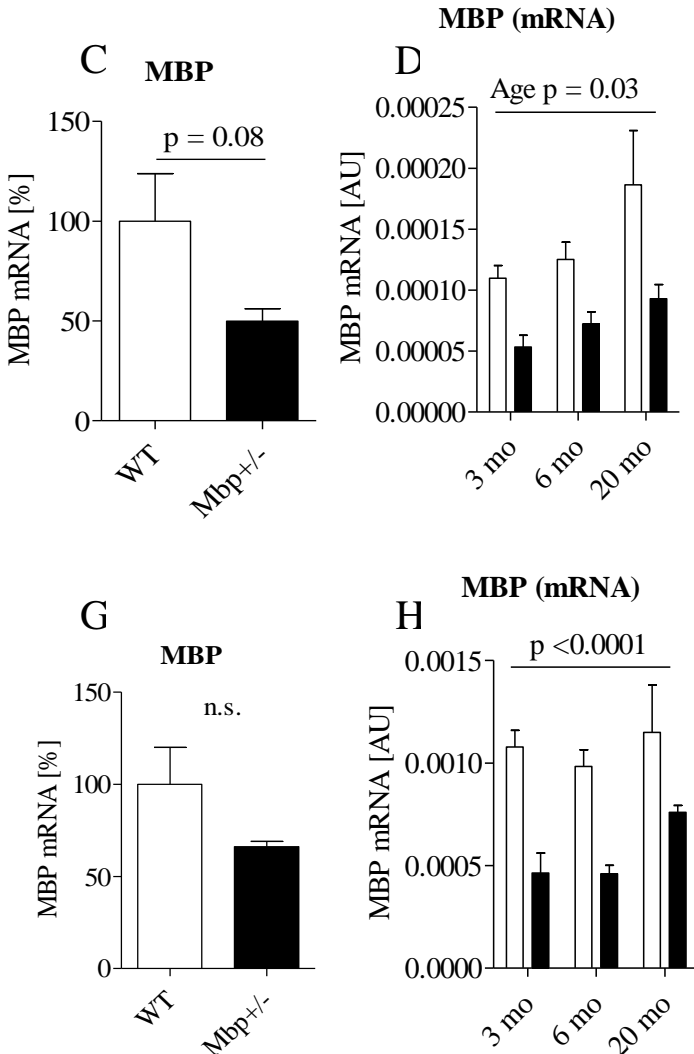

Figure 7: Quantification of $\boldsymbol{M b p}$ mRNA. Percentage of $M b p$ mRNA, normalised to $18 s$ mRNA in PFC(A-D) and brainstem (E-H) at 3 months (A, E), 6 months (B,F), 20 months $(\mathrm{C}, \mathrm{G})$ of age. The data are expressed in percentage of the average wild-type \pm SEM; Mbp+/- $\mathrm{n}=4$, wild-type $\mathrm{n}=4$, Mann-Whitney test. Lifespan amount of $M b p$ mRNA, normalised to $18 \mathrm{~s}$ mRNA, in $P F C(\mathrm{D})$ and brainstem $(\mathrm{H})$. The data are expressed in arbitrary unit (AU) $\pm \mathrm{SEM} ; \mathrm{Mbp}+/-\mathrm{n}=4$, wild-type $\mathrm{n}=4$, two-way ANOVA. 
MBP undergoes a peculiar translational process, so protein quantification via western blot was required to confirm whether the mRNA level was representative of the protein level.

Indeed, at 3 and 6 months Mbp+/- mice expressed $\sim 50 \%$ of the $M B P$ amount present in wild-type. This held true for both PFC (3 months, $\mathrm{p}=0.03$, Mann-Whitney; 6 months, $\mathrm{p}$ $=0.01$, Mann-Whitney; wild-type $\mathrm{n}=4, \mathrm{Mbp}+/-\mathrm{n}=4$; Fig. 8 A, B) and brainstem (3 months, $\mathrm{p}=0.007$, Mann-Whitney; 6 months, $\mathrm{p}=0.03$, Mann-Whitney; wild-type $\mathrm{n}=4$, Mbp+/- n=4; Fig. 8 E, F). Unexpectedly, at 18 months we observed a discrepancy between the protein level and the mRNA data. In the brainstem, the amount of MBP protein was almost comparable between genotypes (Mann-Whitney, $\mathrm{p}=0.2$, wild-type n=4, Mbp+/- n=4; Fig. $8 \mathrm{G})$, whereas it was drastically reduced $(<50 \%)$ in the $P F C$ of Mbp +/- vs wild-type (Mann-Whitney, p = 0.03; wild-type n=4, Mbp+/-n=4, Fig. 8 C). Thus, it appears that, upon ageing, $P F C$ and brainstem respond differently to alteration in MBP gene-dosage.

Independently of the genotype, $M B P$ protein in the $P F C$ increased during adulthood and drastically decreased upon ageing $(\mathrm{p}<0.0001$, two-way ANOVA, wild-type $\mathrm{n}=4$, $\mathrm{Mbp}+/-\mathrm{n}=4$ per time point Fig. $8 \mathrm{D})$. In the brainstem the highest expression of $M B P$ was detected at 3 months and the amount of protein gradually decreased upon ageing ( $\mathrm{p}<0.0001$, two-way ANOVA, wild-type $n=4, \mathrm{Mbp}+/-\mathrm{n}=4$ per time point, Fig. $8 \mathrm{H}$ ). In summary, MBP expression data and the respective protein quantification suggest a peculiar spatiotemporal pattern of MBP expression. 

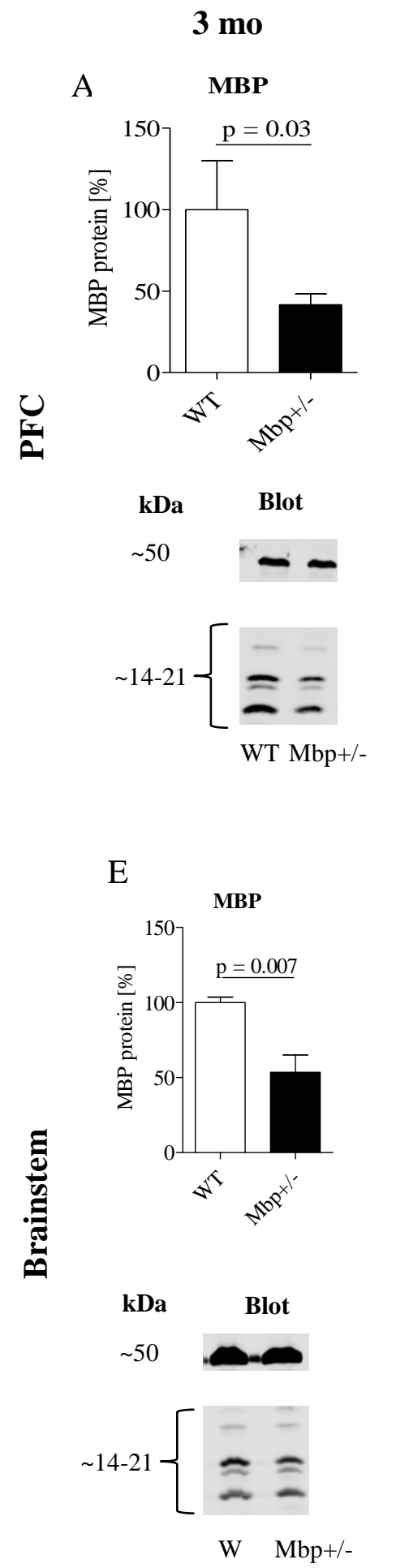
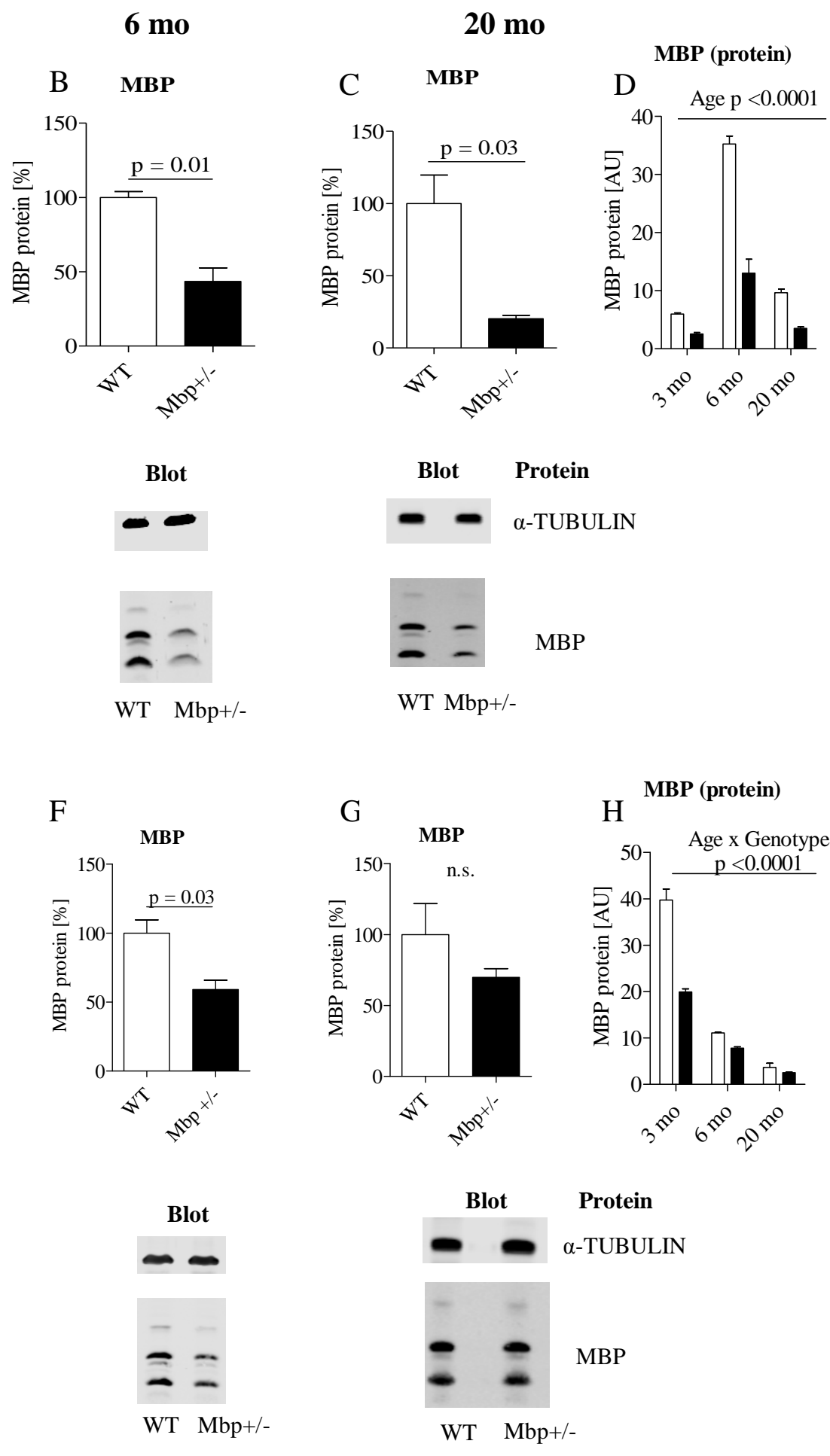

Figure 8: Quantification of $\mathbf{M B P}$ protein. Percentage to average wild-type of $M B P$ protein, normalised to $\alpha$-tubulin, in PFC(A-D) and brainstem (E-H) at 3 months (A, E), 6 months (B,F), 20 months $(\mathrm{C}, \mathrm{G})$ of age. The data are expressed in percentage of the average wild-type \pm SEM; $\mathrm{Mbp}+/-\mathrm{n}=4$, wild-type $\mathrm{n}=4$, Mann-Whitney test. Lifespan amount of MBPprotein, normalised to $\alpha$-tubulin, in $P F C(\mathrm{D})$ and brainstem $(\mathrm{H})$. The data are expressed in arbitrary unit (AU) \pm SEM; $\mathrm{Mbp}+/-\mathrm{n}=4$, wild-type $\mathrm{n}=4$, two-way ANOVA. The graphs are accompanied by the respective blot. 


\subsection{The lack of one allele coding for MBP does not have drastic effects on the expression of other myelin-related molecules and on oligodendrocyte number}

To have a preliminary estimation of major effects of MBP reduction on oligodendrocyte number, we quantified $P l p$ and $C n p$ mRNA. It is worth mentioning that also Plp and Cnp mRNAsare reduced in post-mortem brain of schizophrenia patients (Table A appendix) (Hakak, Walker et al. 2001, Tkachev, Mimmack et al. 2003). In both PFC and brainstem, the percentage of $P l p$ was generally comparable between genotypes ( $\mathrm{p}$ $>0.05$, Mann-Whitney, wild-type $\mathrm{n}=4, \mathrm{Mbp}+/-\mathrm{n}=4$ per time point; Fig. 9A-C, E, G). The only exception was an isolated reduction in the brainstem of 6 months in Mbp+/mice compared with wild-type $(p=0.03$, Mann-Whitney, wild-type $n=4, M b p+/-n=4$; Fig. 9F). The percentage of $C n p$ mRNA was totally comparable between genotypes, in both PFC (p >0.05, Mann-Whitney, wild-type $\mathrm{n}=4, \mathrm{Mbp}+/-\mathrm{n}=4$ per time point; Fig. 10A-C) and brainstem ( $>0.05$, Mann-Whitney, wild-type $n=4, M b p+/-n=4$ per time point; Fig. 10E-G).
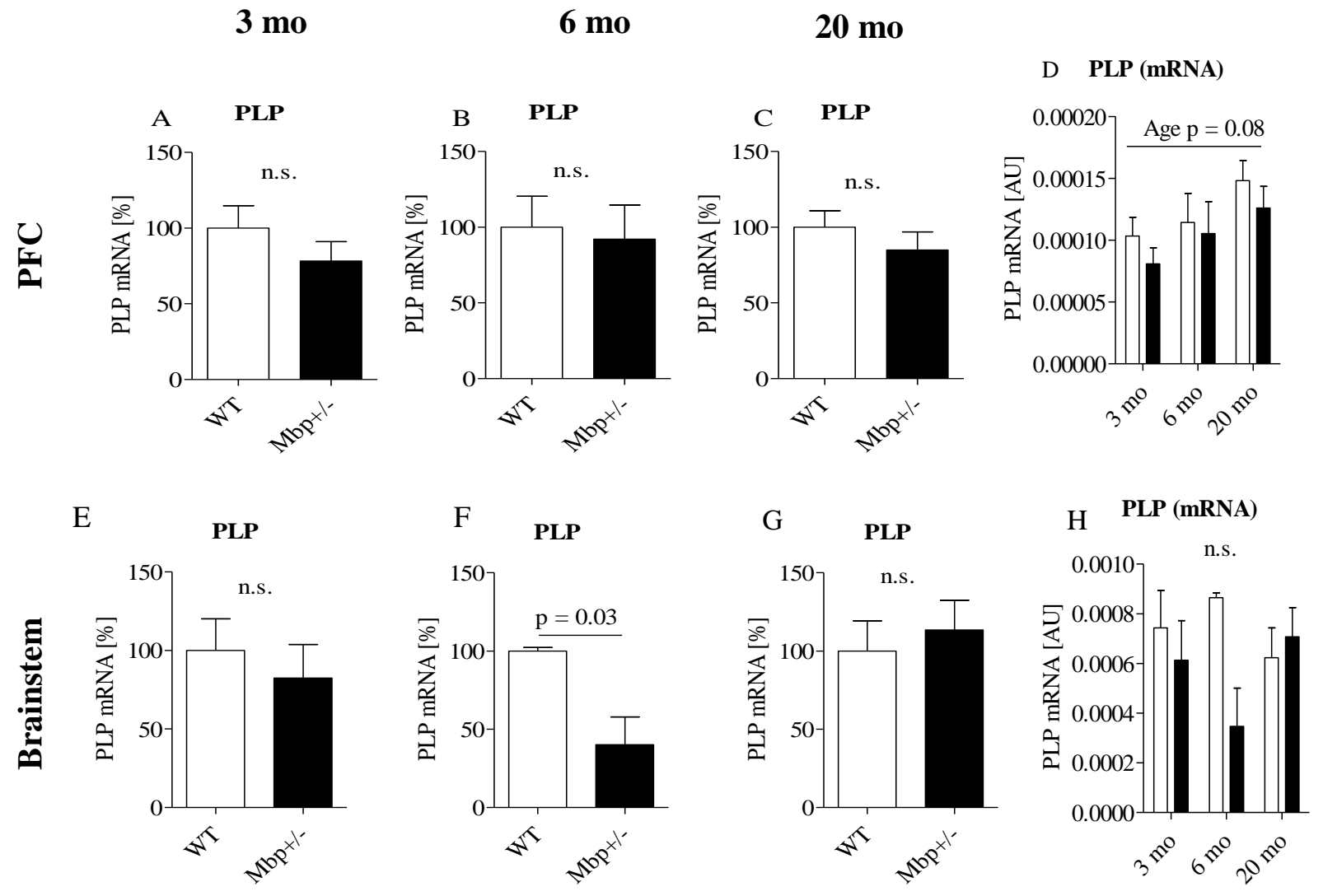

Figure 9: Quantification of $\boldsymbol{P l} \boldsymbol{p}$ mRNA. Percentage of $P l p$ mRNA, normalised to $18 s$ mRNA in PFC (A-D) and brainstem (E-H) at 3 months (A, E), 6 months (B,F), 20 months (C, G) of age. The data are expressed in percentage of the average wild-type \pm SEM; $M b p+/-n=4$, wild-type $\mathrm{n}=4$, Mann-Whitney test. Lifespan amount of Plp mRNA, normalised to $18 s$ mRNA, in PFC (D) and brainstem $(\mathrm{H})$. The data are expressed in arbitrary unit $(\mathrm{AU}) \pm \mathrm{SEM} ; \mathrm{Mbp}+/-\mathrm{n}=4$, wild-type $\mathrm{n}=4$, two-way ANOVA. 

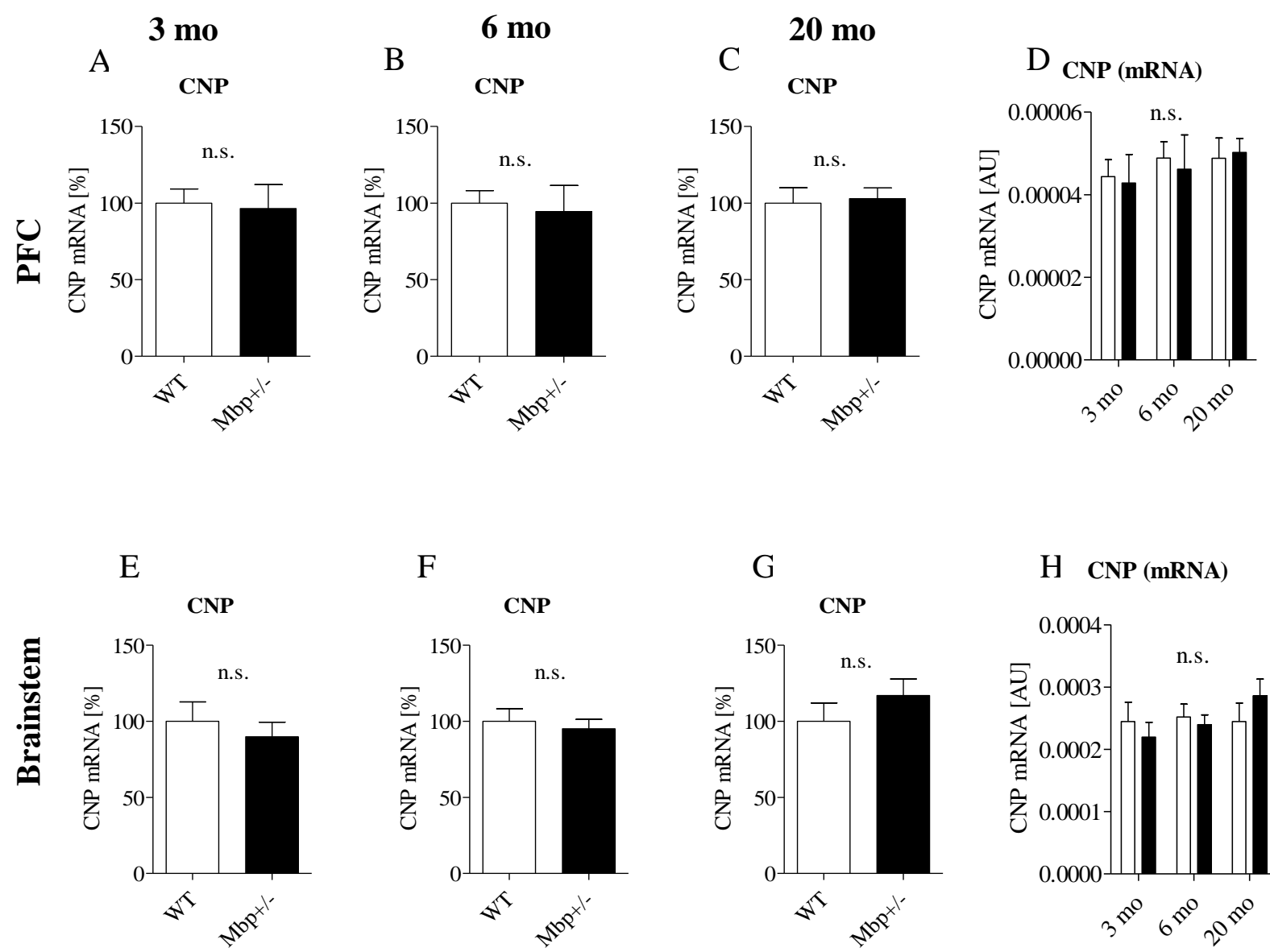

\section{H CNP (mRNA)}

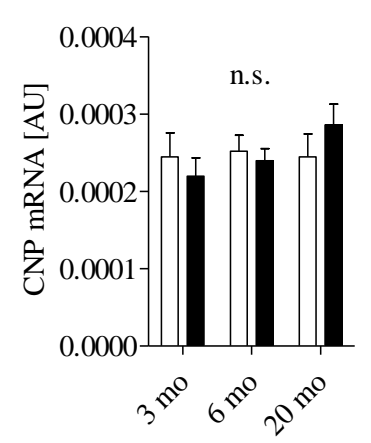

Figure 10: Quantification of Cnp mRNA. Percentage of Cnp mRNA, normalised to $18 \mathrm{~s}$ mRNA in PFC (A-D) and brainstem (E-H) at 3 months (A, E), 6 months (B,F), 20 months (C, $\mathrm{G})$ of age. The data are expressed in percentage of the average wild-type $\pm \mathrm{SEM}$; $\mathrm{Mbp}+/-\mathrm{n}=4$, wild-type $\mathrm{n}=4$, Mann-Whitney test. Lifespan amount of $C n p$ mRNA, normalised to $18 \mathrm{~s}$ mRNA, in PFC (D) and brainstem (H). The data are expressed in arbitrary unit (AU) $\pm \mathrm{SEM}$; Mbp + $\mathrm{n}=4$, wild-type $\mathrm{n}=4$, two-way ANOVA.

Independently of the genotype, the amount of $P l p$ mRNA in the $P F C$ tend to increase upon ageing ( $p=0.08$, two-way ANOVA; $p=0.02$, Jonckheere Terpstra test; wild-type $\mathrm{n}=4, \mathrm{Mbp}+/-\mathrm{n}=4$ per time point; Fig. 9D). No trend was detected in the brainstem ( $\mathrm{p}=$ 0.8, two-way ANOVA; $\mathrm{p}=0.8$, Jonckheere Terpstra test; wild-type $n=4, \mathrm{Mbp}+/-\mathrm{n}=4$ per time point, Fig. 9H).

Cnp mRNA was not affected by ageing in either in the PCF (p $=0.5$, two-way ANOVA; $\mathrm{p}=0.3$, Jonckheere Terpstra test; wild-type $\mathrm{n}=4, \mathrm{Mbp}+/-\mathrm{n}=4$ per time point; Fig. 10D) or in the brainstem ( $\mathrm{p}=0.4$, two-way ANOVA; $\mathrm{p}=0.08$, Jonckheere Terpstra test; wild-type $n=4, M b p+/-n=4$ per time point; Fig. $10 \mathrm{H})$. At the protein level, the percentage of $P L P$ was comparable between groups (Fig.11 A-C, E, F), except for a 


\section{RESULTS}

mild reduction in the brainstem of 3-month old $\mathrm{Mbp}+/$ - vs. wild-type ( $\mathrm{p}=0.03$, MannWhitney, wild-type n=4, Mbp+/- n=4; Fig.11D).

The percentage of $C N P$ protein was generally unchanged in $\mathrm{Mbp}+/-(\mathrm{p}>0.05$, MannWhitney, wild-type $n=4, M b p+/-n=4$ per time point; Fig. $12 \mathrm{~A}, \mathrm{~B}, \mathrm{D}-\mathrm{F}$ ), but it is worth mentioning that at 20 months the percentage of $C N P$ was visibly reduced in the $P F C$ of $\mathrm{Mbp}+/$ - vs. wild-type. Yet this reduction did not reach statistical significance $(\mathrm{p}=0.06$, Mann-Whitney, wild-type $n=4, \mathrm{Mbp}+/-\mathrm{n}=4$; Fig. 12C). Thus, the reduction of MBP seems not to have any major effect on the number of oligodendrocytes. Moreover, $P l p$ mRNA, as much as $M b p$ mRNA, increased upon ageing in the $P F C$ and not in the brainstem. This suggests an area-specific gene expression regulation for members of the compact myelin. MBP diminishment affects only marginally the amount of CNP and PLP proteins. 
3 mo
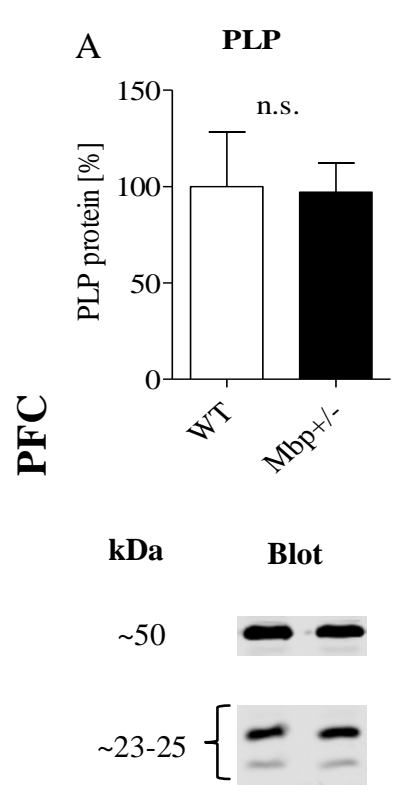

WT Mbp+/-
6 mo
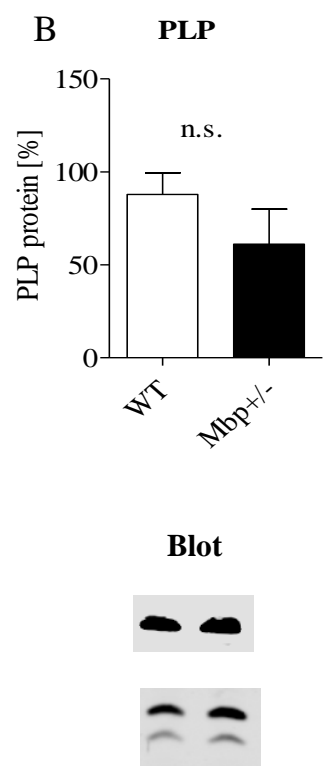

WT Mbp+/-

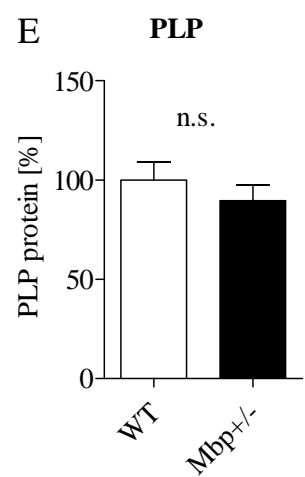

Blot

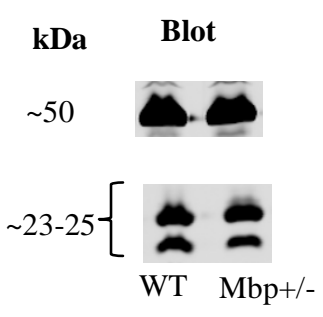

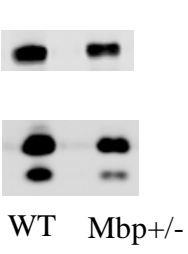

D

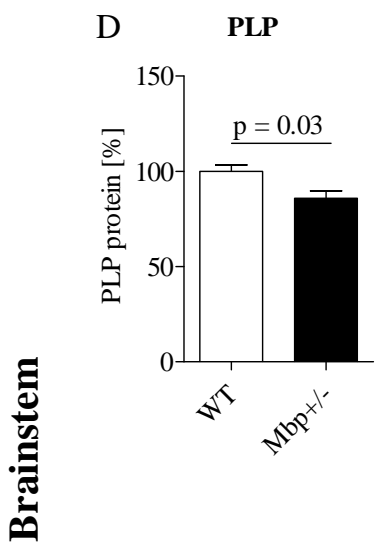

ฮี้

WT Mbp+/-
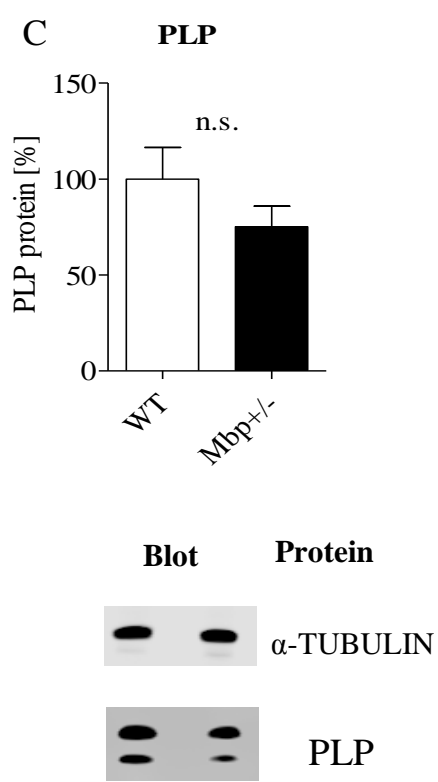

Mbp+/- WT
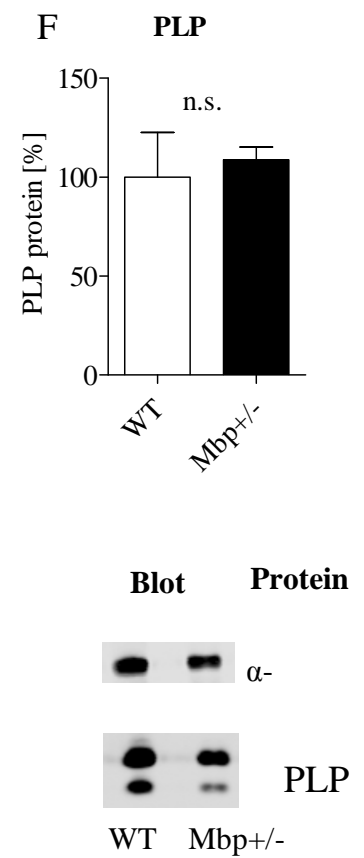

Figure 11: Quantification of $P L P$ protein. Percentage to average wild-type of $P L P$ protein, normalised to $\alpha$-tubulin, in $P F C(\mathrm{~A}-\mathrm{C}$ ) and brainstem (D-F) at 3 months (A, D), 6 months $(\mathrm{B}, \mathrm{E}), 20$ months $(\mathrm{C}, \mathrm{F})$ of age. The data are expressed in percentage to average wild-type \pm SEM; $M b p+/-n=4$, wild-type $n=4$, Mann-Whitney test. The graphs are accompanied by the respective blots. 

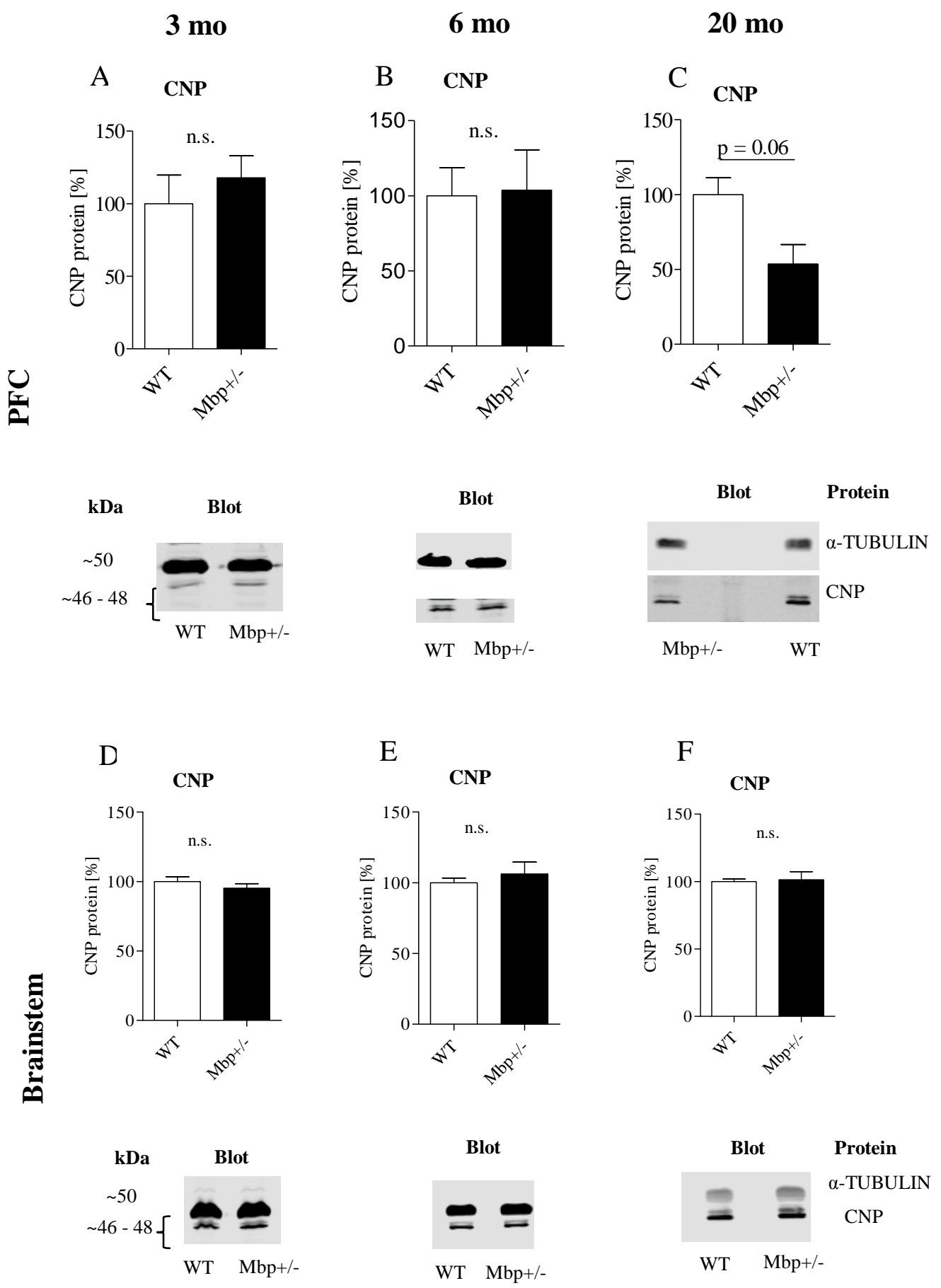

Figure 12: Quantification of $\boldsymbol{C N P}$ protein. Percentage to average wild-type of CNP protein, normalised to $\alpha$-tubulin, in $P F C(\mathrm{~A}-\mathrm{C})$ and brainstem (D-F) at 3 months (A, D), 6 months (B,E), 20 months (C, F) of age. The data are expressed in percentage to average wild-type \pm SEM; $M b p+/-n=4$, wild-type $n=4$, MannWhitney test. The graphs are accompanied by the respective blots. 


\subsection{Mbp+/- showed alteration of white matter tracts}

Several studies with conventional and non-conventional Magnetic Resonance Imaging (MRI) showed white matter anomalies in schizophrenia patients (section 2.4.2). To understand whether and to which extent MBP reduction contributes to alteration detected via in imaging, we ran MRI on Mbp+/- vs. wild-type animals in collaboration with Prof. Boretius.

Three-month and 6-month old animals did not drastically differ from each other with respect to expression data. Since we expected them to be comparable also in MRI, we decided to exclude 3-month old animals from MRI measurements and to focus on mature (6-month old) and senior (18-month old) mice.
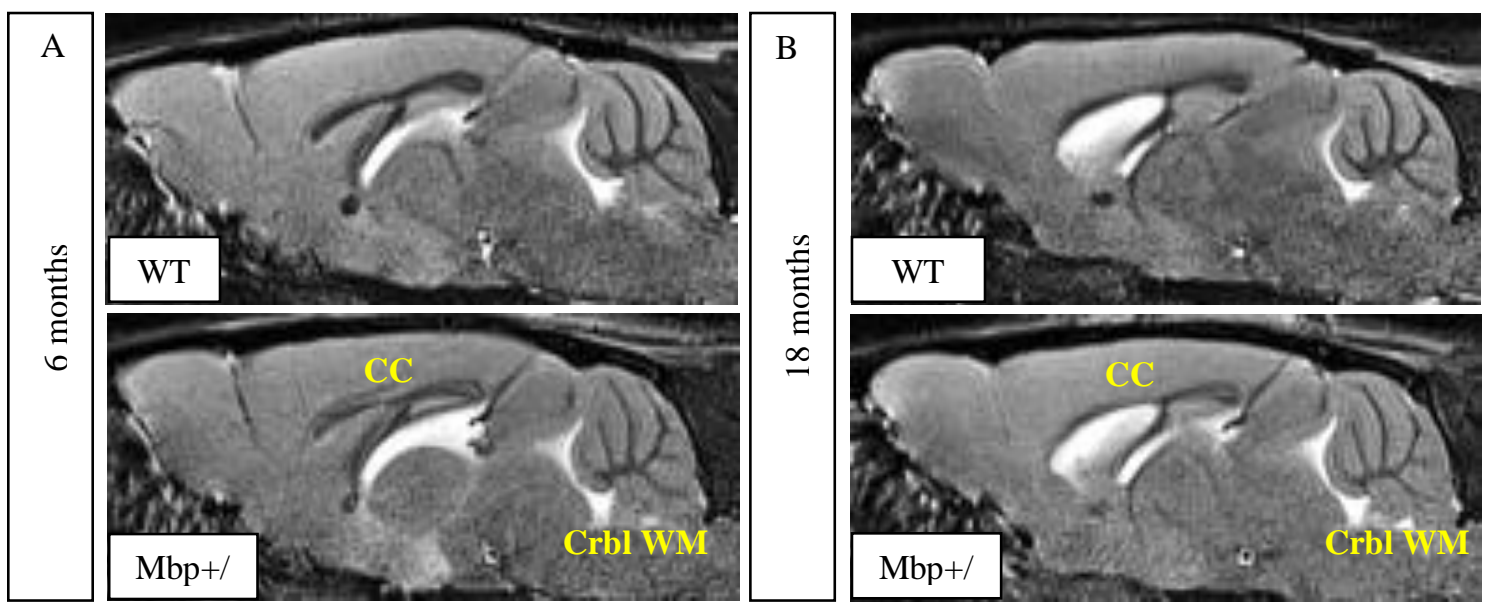

Figure 13: Sagital section from T2-wighted images. Representative panel showing the thinning in the corpus callosum (CC) in Mbp+/- compared with wild-type at 6 months (A) and 18 months (B). A mild contrast difference can be observed also in the cerebellar white matter (Crbl WM).

In collaboration with Prof. Boretius (Klinik für Radiologie und Neuroradiologie, Universitätsklinikum Schleswig-Holstein, Campus Kiel) we ran a comprehensive characterization of $\mathrm{Mbp}+/-$ vs. wild-type mice via MRI.

Volumetric analysis on $\mathrm{Mbp}+/$ - vs. wild-type brains did not reveal any significant difference (Table 3). A qualitative inspection of T2-wighted images pinpointed a minor thinning of the corpus callosum, a general reduction of the white-to-grey matter contrast and a mild alteration in the contrast of cerebellum white matter in 6-month and 18month old Mbp +/- compared with wild-type (Fig. 13). Such differences appeared more 
prominent upon ageing (Fig. 13). Thus, the reduction in MBP does not have a major impact on total brain and ventricle size, but specifically affects the corpus callosum. Such effect seems to worsen upon ageing. To understand whether the changes in the corpus callosum were caused by myelin and fibre alteration, we performed Magnetisation Transfer (MT) imaging.

MT imaging of the corpus callosum revealed $\sim 4 \%$ reduction in Magnetisation Transfer Ratio (MTR), 20\% reduction in Magnetisation Transfer (MT) and $\sim 10 \%$ increase in $\mathrm{T} 1$ in Mbp+/- vs. wild-type (Table 3), at both 6 months and 18 months. This suggested that $\mathrm{Mbp}+/$ - mice presented mild anomalies in the myelinated fibres of the corpus callosum.

Alteration in myelin sheath favours a more disorganised motion of water molecules in the brain tissue. The directionality of the movement of water molecules can be measured via DTI. To confirm MT data, we imaged the corpus callosum also via DTI. However DTI did not mirror the MT data and we could not detect any genotype effect on any of the DTI parameters (Table 3).

Independently of the genotype, we observed that MTR and MT mildly increased upon ageing (Table 3). Moreover, we observed a general age effect on the parameters measured via DTI, i.e. mild increase in Fractional Anisotropy (FA) and a minor decrease of Axial Diffusivity (AD), Radial Diffusivity (RD) and Apparent Diffusion Coefficient (ADC) upon ageing (Table 3).

\subsection{The rostral corpus callosum in $\mathrm{Mbp}+/$ - showed a trend in reduction of myelin thickness}

T2-weighted images and the MT imaging suggested mild abnormalities in the callosal fibres of $\mathrm{Mbp}+/-$, but DTI did not show any genotype-related difference. Moreover, visual inspection of fibre staining in the corpus callosum of 18-month old animals did not reveal any major genotype-related difference (Fig. 14 1vs.2 and 3vs.4).

To explain the controversial MRI readouts and to clarify whether there was a real alteration in myelin ultrastructure in the corpus callosum, we ran conventional Electron Microscopy (EM) in collaboration with Dr. Möbius (Department of Neurogenetics, EM core, MPI of Experimental Medicine, Göttingen). 
Due to the MBP expression data we gathered, we gave priority to the analysis on 18 month old $\mathrm{Mbp}+/$ - vs. wild-type (3 animals per groups). The percentage of nonmyelinated axons (Fig. 14A) and the size of the inner tongue (t-ratio, Fig. 14B) were comparable between genotypes. Both $\mathrm{Mbp}+/-$ and wild-type mice showed occasional widening of the innermost myelin growth zone (t-ratio). This event appeared to be slightly more frequent in $\mathrm{Mbp}+/-$, but the difference did not reach statistical significance $(\mathrm{p}=0.24$, t test, $\mathrm{Mbp}+/-\mathrm{n}=3$, wild-type $\mathrm{n}=3$; Fig. 14C). The quantification of myelin thickness (g-ratio) pinpointed a thinning the myelin sheath in $\mathrm{Mbp}+/$ - animals compared to wild-type ( $\mathrm{p}=0.051$, $\mathrm{t}$ test, Mbp+/- $\mathrm{n}=3$, wild-type $\mathrm{n}=3$; Fig. 14E, F). This phenotype seemed to be affecting mainly axons with a diameter $<0.6 \mu \mathrm{m}(\mathrm{p}=0.005$, t test, $\mathrm{Mbp}+/-$ $\mathrm{n}=3$, wild-type $\mathrm{n}=3$; Fig. $15 \mathrm{~A}, \mathrm{~B}$ ), without any effect on the axons with a diameter $>0.6 \mu \mathrm{m}(\mathrm{p}>0.05$, $\mathrm{t}$ test, Mbp+/- $\mathrm{n}=3$, wild-type $\mathrm{n}=3$; Fig. $15 \mathrm{C}$ ). It is important to note that we detected a highly significant inter-individual variability in the parameters we quantified. This is perfectly in line with the normal physiological variability among animals. On the other hand, this could make our analysis statistically underpowered for detecting more subtle phenotypes. 
Table 3. Magnetic Resonance Imaging results

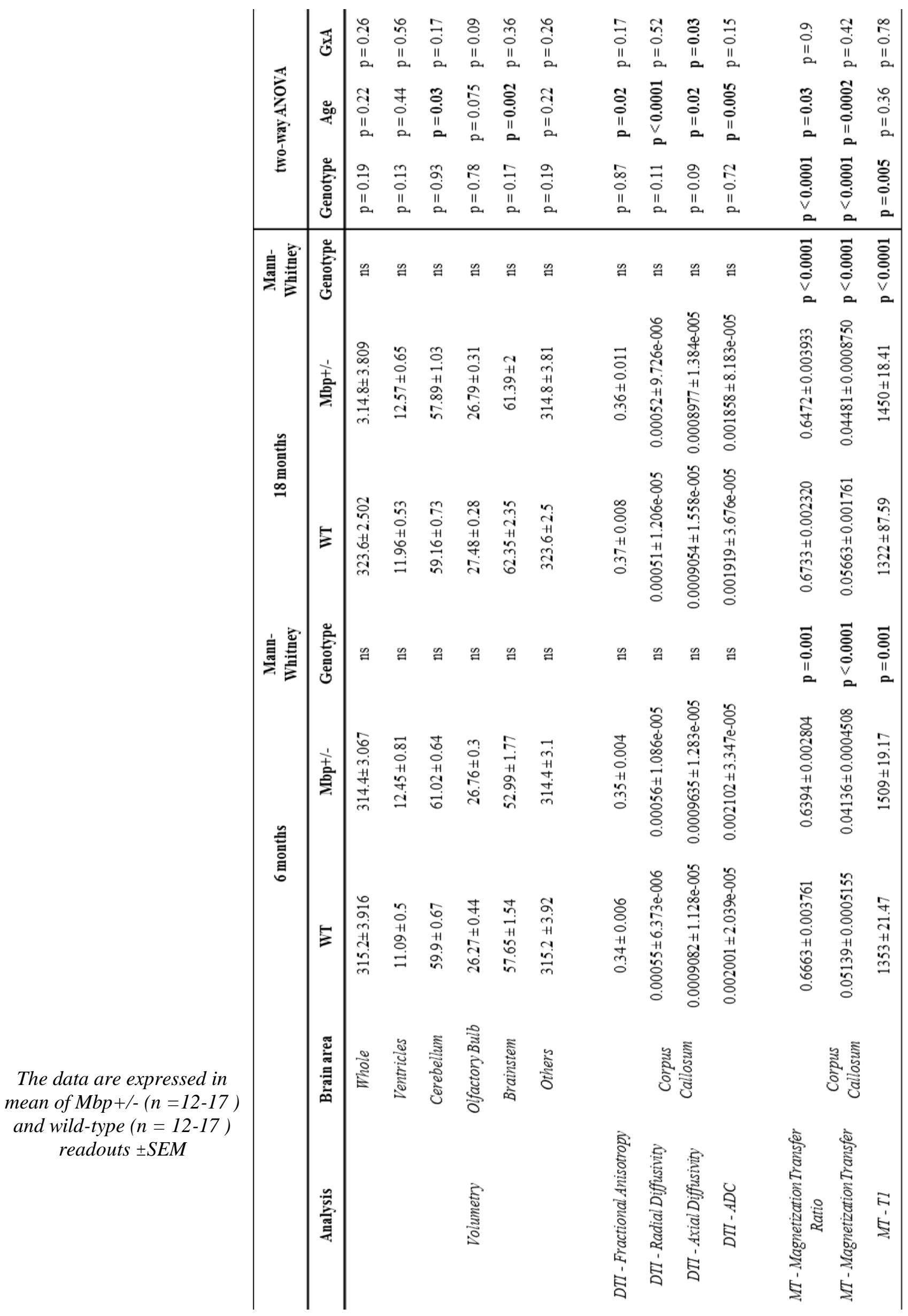



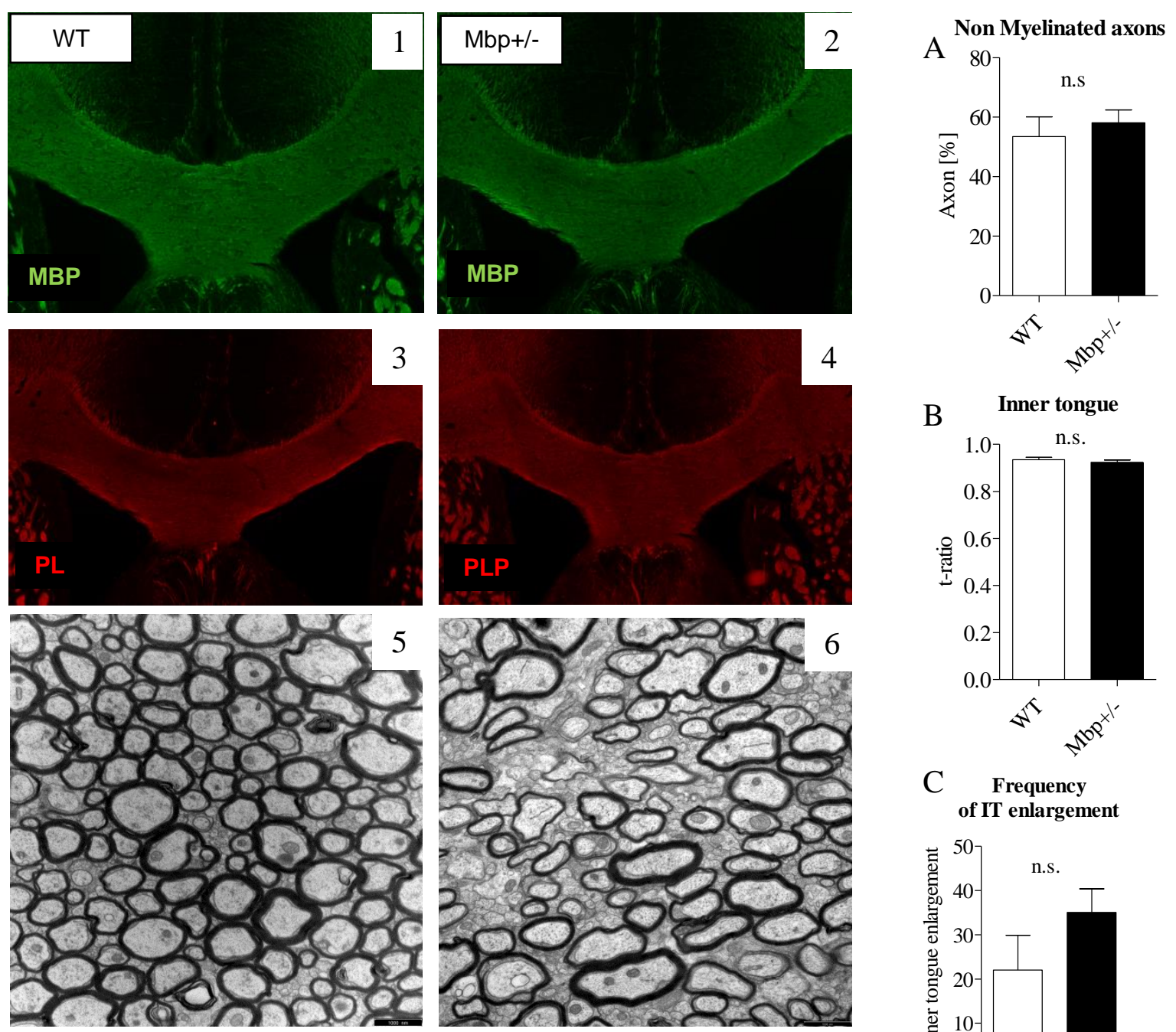

C Frequency of IT enlargement

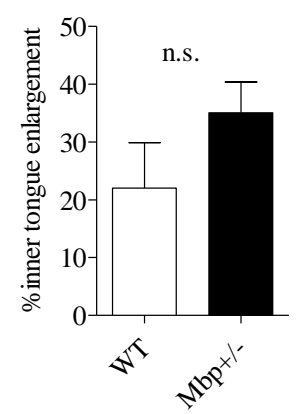

\# cut-off $=$ avg WT- $2 *$ StDev
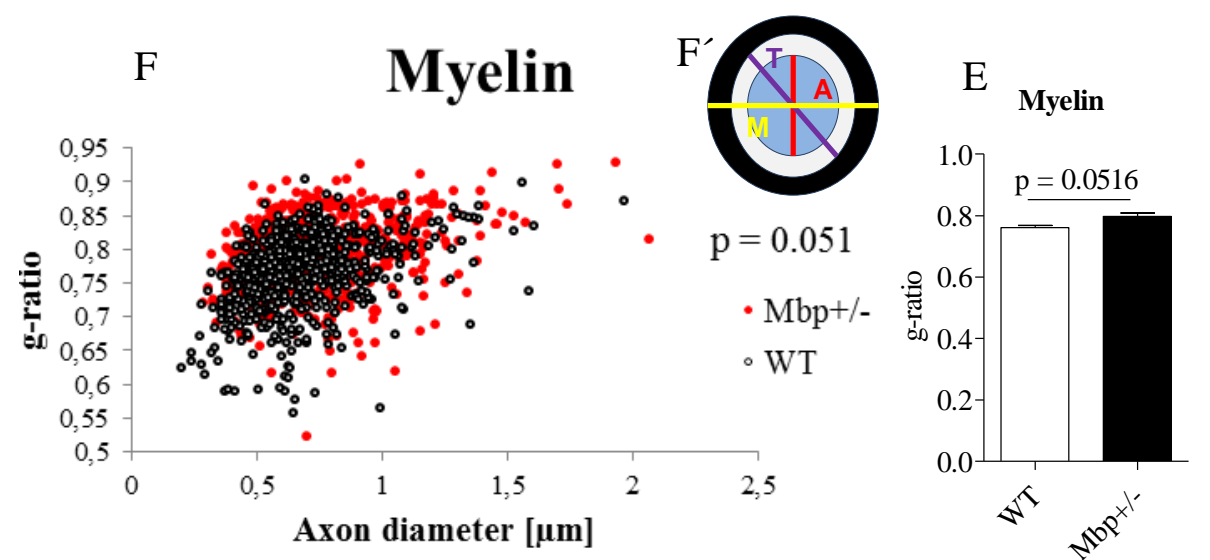

$\mathrm{D}$

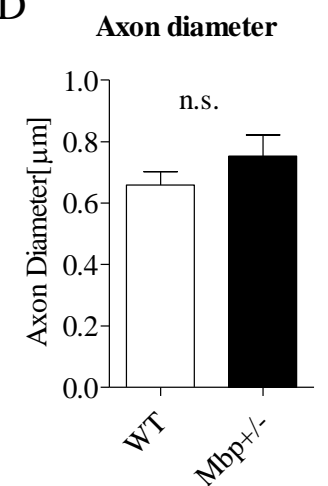

Figure 14: Analysis of myelin ultrastructure. Representative staining for MBP and PLP in the rostral corpus callosum of Mbp+/- vs. Wild-type mice (panel 1-4). Representative EM micrograph of Mbp+/vs. wild-type (panel 5,6; magnification 12000X, scale bar 1000nm). Quantification of non-myelinated axons (A), inner tongue size (B), frequency of inner tongue enlargement (C), axon diameter (D), myelin thickness (E,F). Referring to $\mathrm{F}^{\prime}$, g-ratio has been calculated as $\mathrm{A} /[\mathrm{A}+(\mathrm{M}-\mathrm{T})]$; t-ratio has been calculated as A/T. The data in the bar graphs are expressed as mean \pm SEM, Mbp $+/-n=3$, wild-type $\mathrm{n}=3$. Statistical significance was estimated via Stundent's $\mathrm{t}$ test. 
A Myelin in small caliber axons

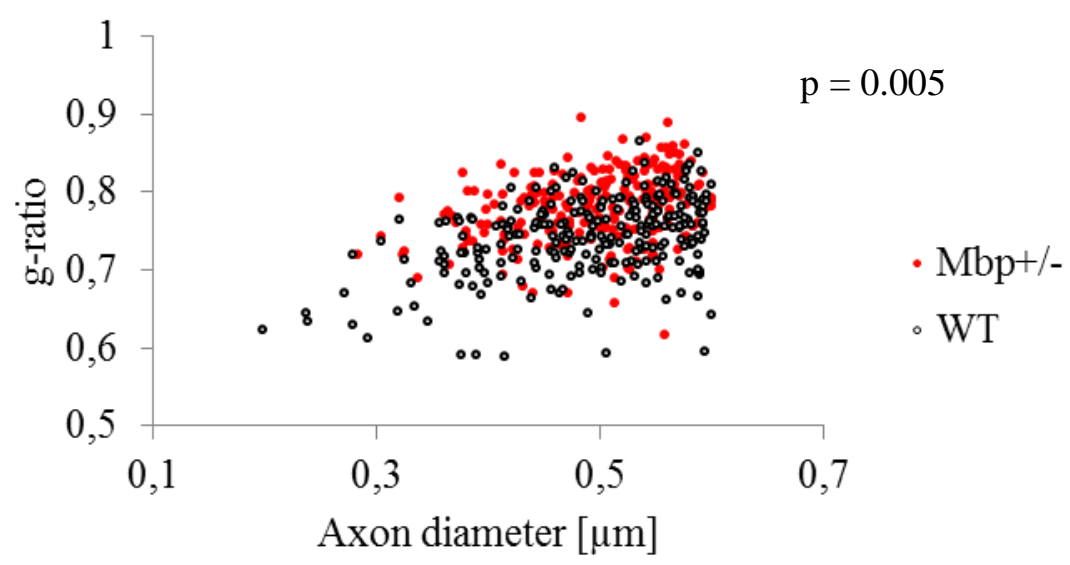

B $\quad$ Myelin

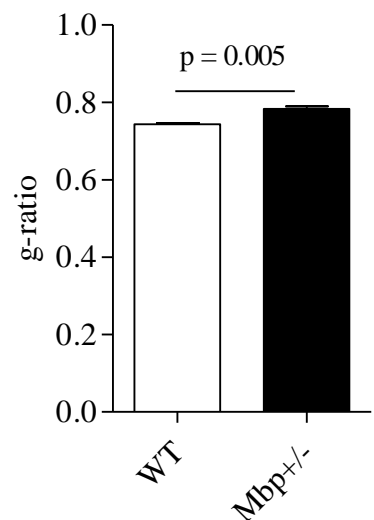

C Myelin big diameter axons (>0.6um)

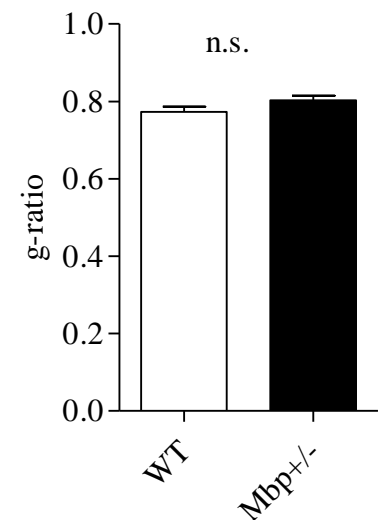

Figure 15: Analysis of myelin thickness in axon subgroups. Myelin thickness in small diameter axons (A, B) and bigger diameter axons $(\mathrm{C})$. The data in the bar graphs are expressed as mean \pm SEM Mbp $+/-n=3$, wild-type $n=3$. Statistical significance was estimated via Stundent's t test. 
A

Interindividual variablity

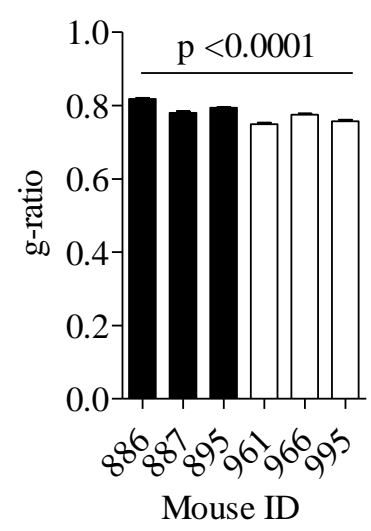

Inner tongue Interindividual variability

C

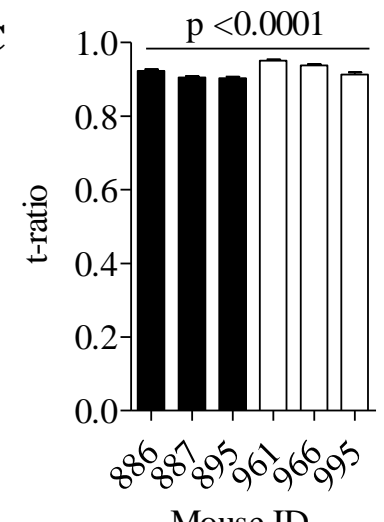

B

Axon
Interindividual variablity

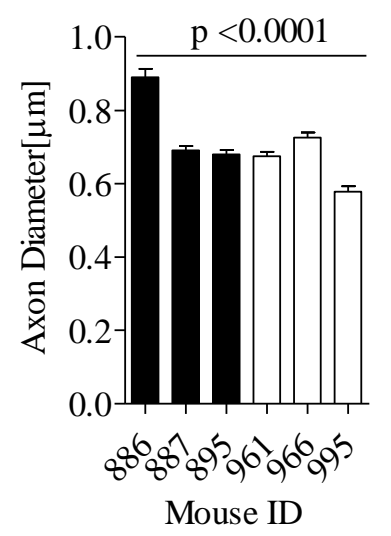

Non myelinated axons Interindividual variability

D

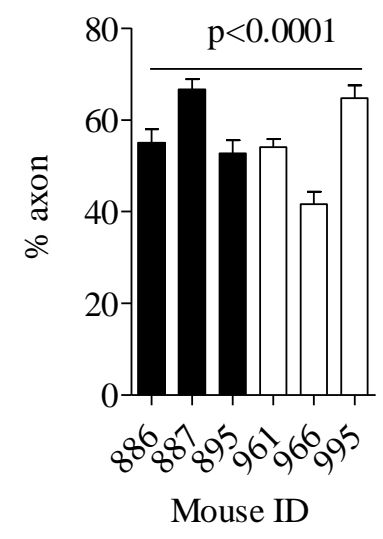

Figure 16: Inter-individual variability in the parameter measured via EM. Comparison among each single mouse in relation to myelin thickness (A), axon diameter (B), inner tongue size (C) and percentage of non-myelinated axons (D). The data are expressed as mean \pm SEM and the statistical significance was estimated via one-way ANOVA.

In summary, ultrastructural analyses of myelin in the rostral corpus callosum support MT readouts and suggest that the contrast alteration in T2-weighted images could be partially due to myelin abnormalities. 


\subsection{Mbp+/- showed alteration of cortical and callosal metabolism}

In parallel to morphological changes, alteration in brain metabolism and neurotransmitters is also documented in schizophrenia patients (Port and Agarwal 2011, Kraguljac, Reid et al. 2012, Rowland, Kontson et al. 2013). Thus we wondered if the reduction in MBP could cause any of these changes. Metabolic data could also help to interpret the discordant results in MRI.

In collaboration with Prof. Boretius (Klinik für Radiologie und Neuroradiologie, Universitätsklinikum Schleswig-Holstein, Campus Kiel) we ran ${ }^{1}$ NMR Spectroscopy in cortex, corpus callosum and hippocampus. Cortex and corpus callosum were chosen because they showed genotype-related differences in all the analyses reported until now. The hippocampus was also included because of the several evidences of its metabolic alteration in schizophrenia(Port and Agarwal 2011). In 6-month old mice we observed increase in neurotransmitters GABA $(+13 \%)$ and Glutamate $(+7 \%)$, accompanied by an increase in Taurine $(+6 \%)$ in the cortex of $\mathrm{Mbp}+/$ - compared with wild-type. We detected a subtle increase of myo-Inositol $(+3 \%)$ both in the cortex and in the corpus callosum of Mbp+/- compared with wild-type, which could suggest the presence of a mild gliosis (Table 4). Upon ageing (18 month) some of these metabolic alterations became even more pronounced. In the cortex of $\mathrm{Mbp}+/$ - mice we observed an increase in Glutamate $(+11 \%)$, in myo-Inositol $(+10 \%)$, in Taurine $(+13 \%)$ and in tCreatine $(+10 \%)$ (Table 4), that could give further evidences for glia activation. In the corpus callosum we detected increase of myo-Inositol $(+12 \%)$ and increase of Taurine $(+7 \%)$ (Table 4). No significant genotype effects were detected in the hippocampus.

Independently of the genotype, an effect of ageing on brain metabolites was detected: an increase of glutamine in cortex, corpus callosum and hippocampus; a reduction of glutamate, GABA, Taurine in the cortex; a trend in the increase of myo-Inositol, an increase of choline and tCreatine in the corpus callosum; an increase of tCreatine in the hippocampus (Table 4).

Taken together the data suggest that the reduction in MBP leads to alteration in cortical and callosal metabolism, specifically in metabolites involved in glial function and neurotransmission. 
Table 4. Magnetic Resonance Spectroscopy

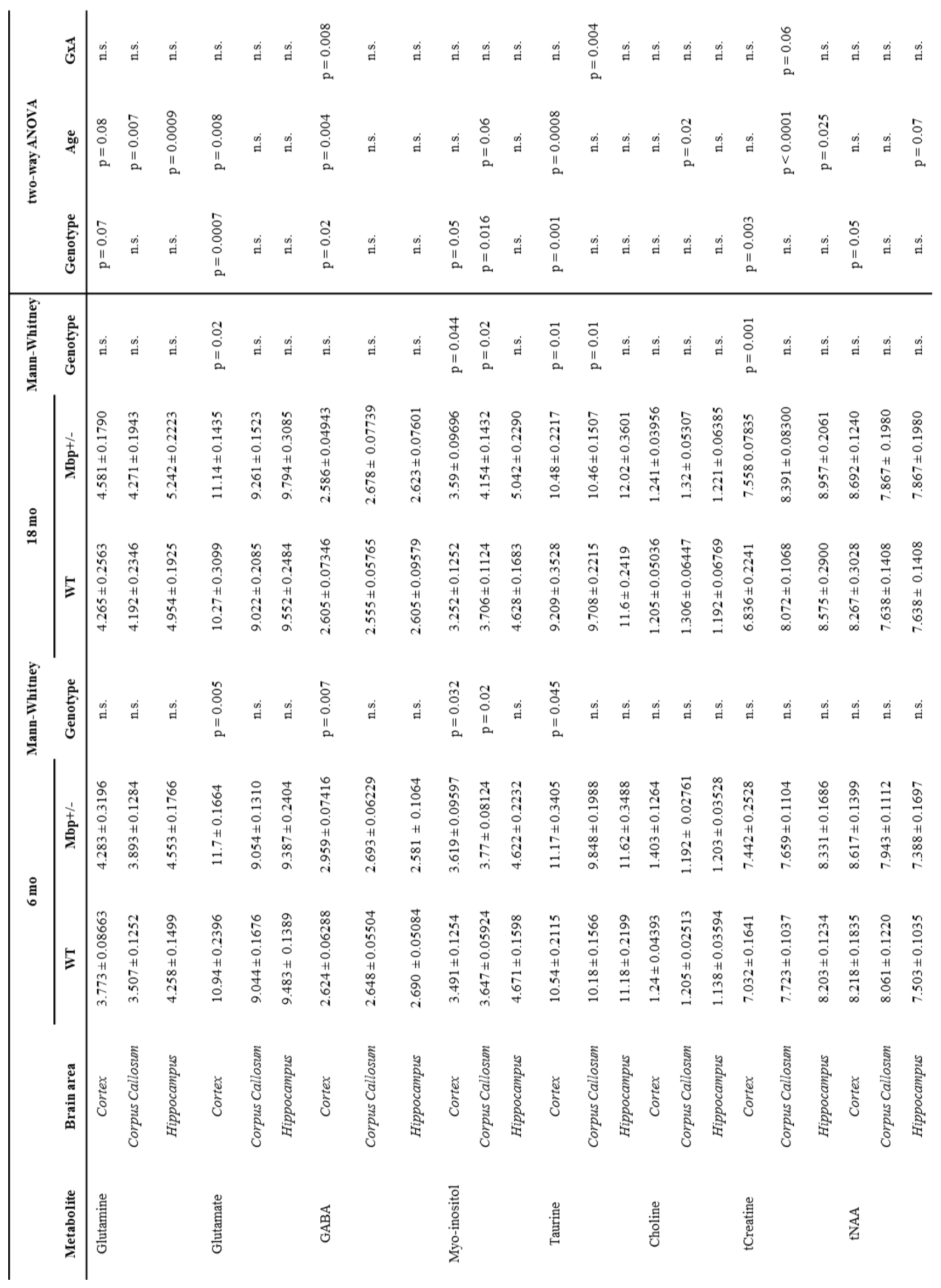




\subsection{MBP reduction led to an increase in the number of Iba1+ cells in the rostral corpus callosum of $\mathrm{Mbp}+/-$ compared with wild-type}

The metabolic alteration detected via ${ }^{1}$ NMR spectroscopy suggested glial activation in the cortex and in the corpus callosum of $\mathrm{Mbp}+/$ - mice compared with wild-type $(\mathrm{p}=0.02$, two-way ANOVA, Mbp+/- $\mathrm{n}=10-18$, wild-type $\mathrm{n}=14-15$; Fig. 17 A). myoInositol increase is often a signature of gliosis (Taylor, Selvaraj et al. 2009)and since we confirmed a mild ultrastructural alteration of myelin in $\mathrm{Mbp}+/-$, we wondered whether the increase in myo-Inositol was associated with changes in microglia number.

We stained brain sections of 14-month old animals (time point in between the two ${ }^{1} \mathrm{NMR}$ spectroscopy measurements) for the microglia marker ionized calcium-binding adapter molecule 1 (IBA1) and we detected almost 30\% increase of Iba1+ cells in the rostral corpus callosum of $\mathrm{Mbp}+/$ - compared with wild-type $(\mathrm{p}=0.005$, two-tailed $\mathrm{t}$ test, Fig. 17B, C).Taken together, the reduction in MBP leads to mild myelin alterations,
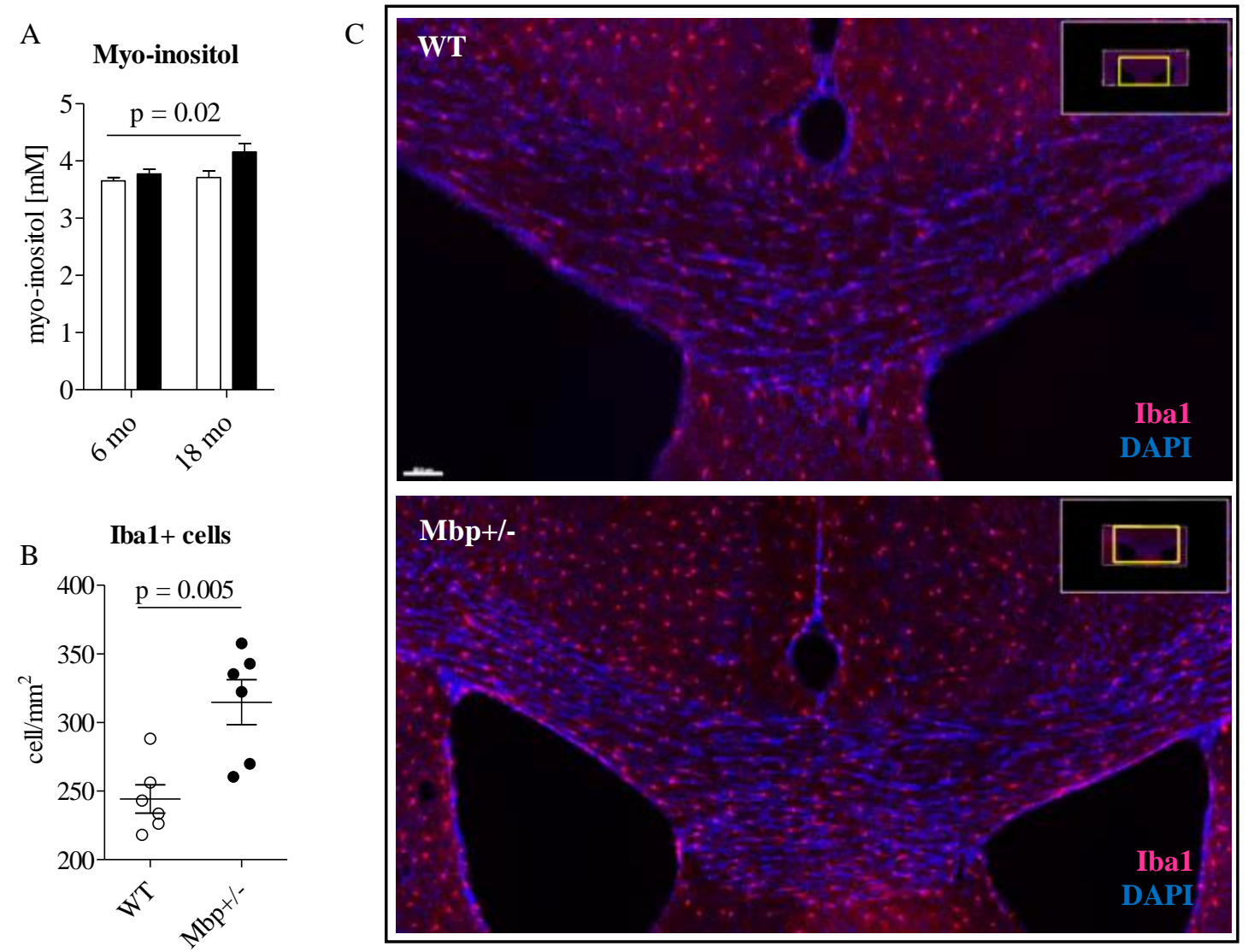

Figure 17: Signs of gliosis in the corpus callosum of Mbp+/- mice. Increase in myoinositol in the corpus callosum of Mbp+/- vs. wild-type (A). The data are expressed as mean values \pm SEM; $M b p+/-n=10-18$, wild-type $n=14-15$. Statistical significance was estimated via two-way ANOVA. Iba1+ cells quantification (B) and representative images of Iba1 staining (Iba1 in red, DAPI in blue). The data are expressed as mean values \pm SEM; $\mathrm{Mbp}+/-\mathrm{n}=6$, wild-type $\mathrm{n}=6$. Statistical significance was estimated via Student's $\mathrm{t}$ test. 
which affects cortical and callosal metabolism, leading to a prominent increase in microglia.

\subsection{Mbp+/- mice specifically showed a decrease in pre-pulse inhibition of startle response and in acoustic startle response}

Our ultimate goal was to explore the role of MBP reduction in schizophrenia symptomatology (see section 2.4.1). Consequently, we screened several behavioural domains in young (3-month to 8-month old) animals and senior (>17-month old) animals.

To exclude any gross alteration of basic behaviour, we first characterise motor and sensory functions. This was the necessary requirement for us to proceed with the analysis of more complex behavioural domains afterwards. Abnormal basic function would invalidate any further behavioural test. As expected, Mbp+/- vs. wild-type mice showed comparable and normal basic sensory and motor functions (Table 5).

Anxiety-like behaviour, tested in elevated plus maze and open field, was comparable between $\mathrm{Mbp}+/-$ and wild-type mice (Table 5). The negative symptoms of schizophrenia include depressive-like behaviour and social withdrawal, so we explore these behavioural domains in our animals. Signs of anhedonia, compulsive behaviour or abnormal house behaviour were not detectable (Table 5). Sociability was also comparable between groups. Cognitive impairment is also reported in schizophrenia patients(Walker, Kestler et al. 2004, Aaron T. Beck 2011), so we screened learning and memory abilities in $\mathrm{Mbp}+/$ - compared with wild-type. Social memory and learning abilities were comparable between genotypes (Table 5). Soft-neurological signs are common in schizophrenia patients and one could speculate that they could be associated with mild myelin alteration.

We set up a behavioural test to quantify fine motor functions and we called it "Grasping test". It derived from the combination of "paw preference test"(Collins 1975, Ribeiro, Eales et al. 2013) with the reaching chamber test to check for grasping abilities(Qian, Lei et al. 2010). However, even in this domain, $\mathrm{Mbp}+/$ - and wild-type were comparable. Schizophrenia patients show deficits in screening out irrelevant environmental stimuli, i.e. sensorimotor gating deficit. This is thought to lead to the cognitive fragmentation often reported in patients(Braff, Stone et al. 1978, Braff, Geyer et al. 2001). Since myelin is crucial for the proper synchronisation among different brain areas, we 
wondered whether such subtle abnormalities in myelin could be causative of sensorimotor gating deficits.

We explored this behavioural domain via pre-pulse inhibition (PPI) of startle reflex.

Both mature and senior Mbp+/- mice showed a reduction in PPI when compared with wild-type $(\mathrm{p}=0.01$, repeated measures two-way ANOVA, Fig. 18A;p =0.08, repeated measures two-way ANOVA,Fig. 18C). Mature Mbp+/- showed a reduction trend in baseline acoustic startle response when compared with wild-type; however the difference did not reach statistical significance ( $\mathrm{p}=0.2$, Mann-Whitney test; Fig. 18B). Upon ageing, senior Mbp+/- clearly manifested a reduction in baseline acoustic startle response when compared with wild-type mice ( $\mathrm{p}=0.01$, Mann-Whitney test; Fig. 18D). Body weight was comparable between groups (data not shown).

Taken together, the reduction of MBP has a clear and specific effect on a behavioural domain involved in sensorimotor gating functions; this domain is clearly affected in schizophrenia patients.

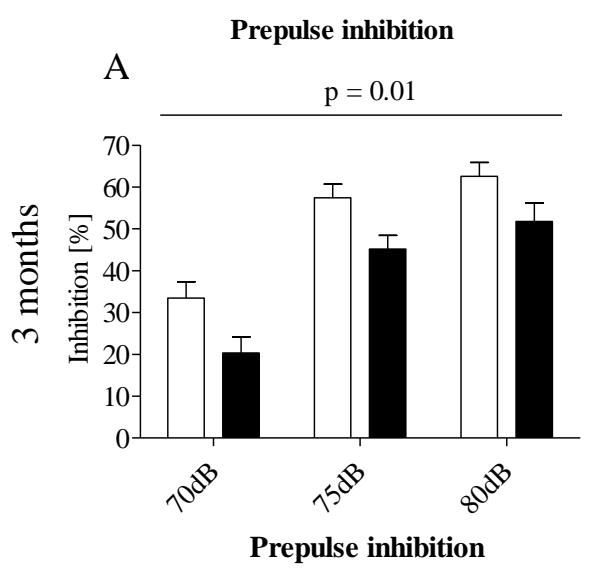

B

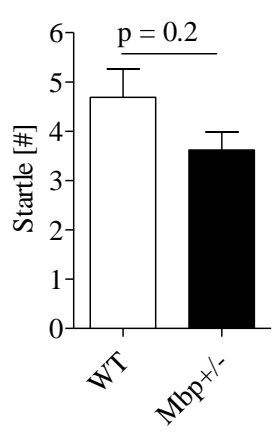

D

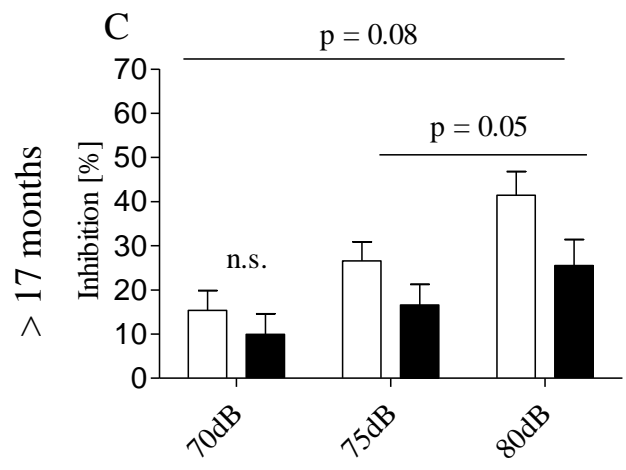

Figure 18: Deficit in sensorimotor gating in $\mathrm{Mbp}+/-$ detected via PPI and reduction in baseline acoustic startle response. Significant reduction in PPI in both young (A) and senior (C) $\mathrm{Mbp}+/$ - mice (black) when compared to wild-type (white). The data are expressed as mean values \pm SEM; $M b p+/-n=34$, wildtype $n=22$. Statistical significance was estimated via repeated measure two-way ANOVA. Baseline of the acoustic startle response in both young (B) and senior (D) $\mathrm{Mbp}+/$ - vs. wild-type mice. The data are expressed as mean values \pm SEM; $\mathrm{Mbp}+/-\mathrm{n}=34$, wild-type $\quad n=22 . \quad$ Statistical significance was estimated via Mann-Whitney test. 


\begin{tabular}{|c|c|c|c|c|c|c|c|}
\hline \multirow[t]{2}{*}{ Behavioral Test } & \multirow[t]{2}{*}{ Behavioral domain } & \multicolumn{3}{|c|}{$\begin{array}{l}3 \mathrm{mo} \\
\text { (wild-type } \mathrm{n}=12-22 ; \mathrm{Mbp}+/-\mathrm{n}=12-34 \text { ) }\end{array}$} & \multicolumn{3}{|c|}{$\begin{array}{l}17 \text { months } \\
\text { (wild-type } \mathrm{n}=12-22 ; \mathrm{Mbp}+/ \mathrm{n}=16-33 \text { ) }\end{array}$} \\
\hline & & WT & $\mathrm{Mbp}+/-$ & p value & WT & Mbp +/- & p value \\
\hline $\begin{array}{l}\text { Elevated plus maze (preference for open } \\
\qquad \text { arms }[\%] \text { ) }\end{array}$ & Anxiety & $31 \pm 5$ & $31,28 \pm 4,774$ & n.s. & - & - & - \\
\hline Open field (total distance $[\mathrm{m}]$ ) & Activity & $48 \pm 1,6$ & $47,6 \pm 1,4$ & n.s. & $36,4 \pm 1,4$ & $37,2 \pm 1,5$ & n.s. \\
\hline $\begin{array}{c}\text { Rota-rod } \\
\text { (latency to fall [s], day } 1 \text { and day 2) }\end{array}$ & $\begin{array}{l}\text { Motor function and } \\
\text { equilibrium }\end{array}$ & $\begin{array}{l}123 \pm 15 \\
214 \pm 18\end{array}$ & $\begin{array}{l}140 \pm 17 \\
208 \pm 15\end{array}$ & n.s. & $81 \pm 15$ & $96 \pm 10$ & n.s. \\
\hline Grip strength [p] & Muscles strength & $142 \pm 3$ & $139 \pm 2$ & n.s. & - & - & - \\
\hline Hot plate (latency to lick [s]) & Heat/pain perception & $15 \pm 1$ & $16 \pm 1$ & n.s. & - & - & - \\
\hline Hole board (1 day) & Explorative behavior & $5 \pm 1$ & $6 \pm 1$ & n.s. & - & - & - \\
\hline Buried food test (digging latency [s]) & Olfaction & $87 \pm 21$ & $74 \pm 13$ & n.s. & - & - & - \\
\hline Visual Cliff (latency in the "air" [\%]) & Vision & $36 \pm 4$ & $34 \pm 3$ & n.s. & $35 \pm 4$ & $26 \pm 4$ & n.s. \\
\hline Hearing & Hearing & - & - & $\begin{array}{l}\text { Genotype n.s. } \\
\text { Intensity } \\
\mathrm{p}>0.0001\end{array}$ & - & - & $\begin{array}{l}\text { Genotypen.s. } \\
(\mathrm{p}=0.0505) \\
\text { Intensity } \\
\mathrm{p}>0.0001\end{array}$ \\
\hline Prepulse inhibition & Sensorimotor gating & - & - & Genotype $\mathrm{p}=0.01$ & - & - & $\begin{array}{c}75-80 \mathrm{~dB} \\
\text { Genotype } \mathrm{p}=0.05\end{array}$ \\
\hline Startle response & Sensorimotor reflex & $4 \pm 0,6$ & $3,5 \pm 0,4$ & n.s. & $3,4 \pm 0,4$ & $2,3 \pm 0,2$ & 0.01 \\
\hline Marble Burying (Marble buried[\#]) & Impulsivity & $7, \pm 1$ & $7 \pm 1$ & n.s. & - & - & - \\
\hline Morris water maze & Spatial memory & & & & & & \\
\hline Escaping latency $[\mathrm{s}]$ & & - & - & $\begin{array}{l}\text { Day } p<0.0001 ; \\
\text { genotype } p=0.63\end{array}$ & - & - & $\begin{array}{l}\text { Day } p<0.0001 \\
\text { genotype } p=0.69\end{array}$ \\
\hline Probe trial - Time in target zone [\%] & & $41 \pm 2$ & $45 \pm 2$ & n.s. & $47 \pm 2$ & $54 \pm 2$ & 0.03 \\
\hline Escaping latency - Reverse [s] & & - & - & $\begin{array}{l}\text { Day } p<0.0001 \\
\text { genotype } p=0.28\end{array}$ & - & - & $\begin{array}{l}\text { Day } p<0.0001 \\
\text { genotype } p=0.17\end{array}$ \\
\hline $\begin{array}{c}\text { Probe trial Reverse - Time in target zone } \\
{[\%]}\end{array}$ & & $33 \pm 2$ & $33 \pm 2$ & n.s. & $41 \pm 2$ & $43 \pm 2$ & n.s. \\
\hline $\begin{array}{l}\text { Novel object recognition (novelty } \\
\text { preference }[\%] \text { ) }\end{array}$ & Learning and memory & $59 \pm 3$ & $58 \pm 3$ & n.s. & $57 \pm 5$ & $59 \pm 4$ & n.s. \\
\hline $\begin{array}{l}\text { Three-chambered social test (Sociability } \\
{[\%] \text { ) }}\end{array}$ & Sociability and social memony & $62 \pm 2$ & $63 \pm 2$ & n.s. & - & - & - \\
\hline Sucrose preference [\%] & Anhedonia & $74 \pm 2$ & $74 \pm 2$ & n.s. & - & - & - \\
\hline $\begin{array}{l}\text { LABORAS (Climbing, Grooming, } \\
\text { Locomotion, Drinking, Eating, Scratching, } \\
\text { Circular movements [s]) }\end{array}$ & Homecage behavior & - & - & - & - & - & Genotype n.s. \\
\hline $\begin{array}{l}\text { Grasping test (Paw preference, grasping } \\
\text { score, efficiency index) }\end{array}$ & $\begin{array}{c}\text { Fine motor abilities and } \\
\text { handedness }\end{array}$ & - & - & - & $0.26 \pm 0.04$ & $0.43 \pm 0.03$ & $p=0.003$ \\
\hline Y-maze(Alternationindex $[\#]$ ) & $\begin{array}{c}\text { Spontaneous } \\
\text { alternation/spatial memory }\end{array}$ & $0,7 \pm 0,05$ & $0,8 \pm 0,03$ & n.s. & - & - & - \\
\hline
\end{tabular}




\section{DISCUSSION}

The aim of my $\mathrm{PhD}$ project was to explore the consequences of myelin-related abnormalities in schizophrenia. Whether myelin alteration is cause or consequence of the pathology it is not clear, but several studies indicate a major role of the myelinrelated pathway in the course of the disease (Goudriaan, de Leeuw et al. 2014, Haroutunian, Katsel et al. 2014). The molecular mechanism leading to myelin anomalies in schizophrenia is not defined; however post-mortem studies revealed a general reduction of several myelin-related proteins (and mRNA), like MBP, suggesting a contribution of oligodendrocyte and myelin to the pathology (Hakak, Walker et al. 2001, Flynn, Lang et al. 2003, Dracheva, Davis et al. 2006).

As major protein of the CNS compact myelin, MBP is one of the most intriguing candidates to study. Yet nobody, until now, extensively analysed the meaning of MBP reduction in the context of schizophrenia.

We extensively characterised a largely unexplored mouse model, heterozygousshiverer (here called $\mathrm{Mbp}+/-$ ). Specifically, we wondered whether the lack of one $M b p$ allele (Barbarese, Nielson et al. 1983) caused any schizophrenia-like phenotype that might have been overlooked in the past.

From post-mortem studies it is not possible to infer at which stages of the disease the changes in MBP expression appeared. Moreover, myelin is a very stable structure and the altered expression of its structural components in animal models has often disclosed a phenotype only upon ageing (Aggarwal, Yurlova et al. 2011, Hagemeyer, Goebbels et al. 2012). Thus, we broaden our analysis and we explored the effect of MBP reduction in young, mature and senior animals, to understand the consequence of MBP reduction at young and older age.

We were able to prove that, indeed, $50 \%$ of MBP is detrimental for the CNS and lead to subtle, yet pathological, phenotypes. We showed that MBP presents a specific spatiotemporal expression pattern and its reduction only mildly affects other two important myelin proteins, i.e. CNP and PLP, and the oligodendrocyte number. Yet, MBP diminishment alters cortical and callosal metabolism and varies fibre structures in the corpus callosum, specifically affecting myelin thickness in small calibre axons (diameter $<0.6 \mu \mathrm{m}$ ) of senior mice. These changes are likely to be associated with the 
increase microgliosis. These subtle biological phenomena induce and orchestrate the abnormal sensorimotor gating and the worsening of the startle response to acoustic stimuli.

The reduction of MBP, at both protein and mRNA level, was the necessary prerequisite for my $\mathrm{PhD}$ project. Moreover, it was necessary to estimate the impact of MBP reduction on the other most abundant myelin-related mRNA and proteins of the CNS, as a proxy of oligodendrocyte number and indication of potential more prominent myelin damage.

Barbarese et al. in the early eighties showed a $\sim 50 \%$ reduction of MBP (mRNA and protein) in total brain and purified myelin of 15-day and 90-day Mbp+/- mice compared with wild-type (Barbarese, Nielson et al. 1983). Besides the reduction of MBP, no other myelin phenotype was detected (Barbarese, Nielson et al. 1983).

In contrast to Barbarese's data, Cammer et al reported that the reduction in MBP in $\mathrm{Mbp}+/$ - mice was also accompanied by a mild diminishment of PLP (Cammer 1982). These controversial observations suffered also from other two main limitations: quantifications were run (1) on the whole brain and (2) at one single time point.

Thus, life-span observation of MBP, PLP, and CNP expression in subregions of the Mbp +/- mouse brain was missing.

We explored the effect of missing one $M b p$ allele (1) at 3 time points (2) in two specific brain areas (PFC and brainstem). The lifespan of mice varies between 1.3 to 3 years, depending on the mouse strain (Comfort 1959), so we selected to run mRNA and protein quantification on young (3 months), mature (6 months) and senior (>17 months) animals. The selection of PFC and brainstem ensured an estimation of the MBP expression in grey vs. white matter and, in parallel, in brain area highly affected in schizophrenia (Perlstein, Carter et al. 2001) and related to high cognitive functions $(P F C)$ in contrast to an area mainly devoted to more basic and vegetative functions (brainstem).

The $\sim 50 \%$ reduction of MBP expression in adult Mbp+/- vs. wild-type was confirmed in both PFC and brainstem and the mild decrease in PLP protein detected by Cammer et al in $\mathrm{Mbp}+/-$ was also partially confirmed (in the brainstem at 3 months). Our results added also a new insight into MBP expression, showing that, upon ageing, PFC becomes much more susceptible than brainstem to the lack of one $M b p$ allele. Already in 1975 it was showed that MBP was strongly reduced in aged human post-mortem 
PFC(Ansari and Loch 1975), suggesting a major effect of ageing on frontal myelination. However no other studies reported such major reduction in MBP protein in $\mathrm{Mbp}+/$ - mice, even in presence of an almost physiological amount of Mbp mRNA.

Studies on developmental process highlighted a specific "spatiotemporal pattern" of myelination and MBP expression, both in rodents and primates. Specifically, myelination process initiates in the caudal part of the brain, proceeds towards the temporal lobe and ends in the frontal areas (Delassalle, Zalc et al. 1981, Kanfer, Parenty et al. 1989, Lebel, Gee et al. 2012, Miller, Duka et al. 2012). Moreover, it has been observed that human myelination in the frontal areas of the brain follows a bell-shape trend, increasing to a maximum peak in late-adulthood and decreasing during the process of ageing (Haroutunian, Katsel et al. 2014). Considering this extreme reduction of MBP protein in the $P F C$, one could speculate that $P F C$ in $\mathrm{Mbp}+/$ - mice ages faster than in wild-type. This hypothesis could be interesting in the context of schizophrenia, which has been also proposed to be a syndrome of accelerated ageing (Kirkpatrick, Messias et al. 2008). The hypothetical accelerated PFC ageing could also explain the reduction trend in CNP protein, exclusively detected in 20-month old Mbp+/- PFC. On the one hand, such reduction could be a first sign of an approaching oligodendrocyterelated pathology, often detected in ageing brain (Weiss, Hammer et al. 2000). On the other hand, since CNP has also been suggested to orchestrate myelin compaction almost in antagonism to MBP (Snaidero, Mobius et al. 2014), it is possible that its reduction might be a homeostatic compensation for the more prominent $(<50 \%)$ MBP diminishment.

These results raise the question on how different brain areas regulate myelination at different period in life. However, my data cannot give a clear and conclusive answer to this question. It appears that, upon ageing, the higher amount of MbpmRNA, does not correlate with the amount of MBP protein. This could be caused by three general mechanisms: (1) the half-life of the $M b p$ mRNA diminishes in the PFC upon ageing, so even an increase in $M b p$ transcription is not able to make up for the high rate of mRNA degradation; (2) the translational machinery for $M B P$ is differently regulated upon ageing in PFC compared to brainstem; (3) the protein stability is reduced and MBP is more prone to degradation. Further studies are required to give a more detailed explanation of this phenomenon. 


\section{DISCUSSION}

The molecular phenotype of $\mathrm{Mbp}+/-$ mice was associated to a quite multifaceted combination of structural and metabolic alterations, detected via structural MRI and MRS. We limited the MRI and MRS measurements to two time points (6 months and $>17$ months), since our expression data did not reveal major differences between 3 months and 6 months animals.

It has been consistently showed that schizophrenia patients often suffer from a reduction in brain volume and ventricular enlargement, mainly due to grey matter loss (Gur, Keshavan et al. 2007, Sun, Maller et al. 2009). However, in our mouse model we failed to detect any major brain atrophy or ventricular enlargement in $\mathrm{Mbp}+/-$ mice compared with wild-type. We cannot completely exclude changes in volume in specific brain regions, but we can confidently state that MBP reduction does not have a major impact on general grey matter volume.

On the other hand we observed alteration in white matter of Mbp+/- mice. In the context of conventional structural MRI, T2-weighted images can give an overview on general alteration in the tissue. Among them, oedema, myelin alteration, tissue damage, gliosis and changes in lipid composition could be detected (Symms, Jager et al. 2004, Barkovich 2005, Tanaka, Iwasaki et al. 2012). Previous studies did not reveal any difference in T2-weighted images in $\mathrm{Mbp}+/$ - vs. wild-type mice (Martin, Hiltner et al. 2006), whereas we unequivocally observed that $\mathrm{Mbp}+/-$ mice present a thinning of the corpus callosum, associated to a reduction of grey matter to white matter contrast. It is noteworthy that a thinning of the corpus callosum has also been reported in schizophrenia patients (Balevich, Haznedar et al. 2015).

The corpus callosum is one of the most highly myelinated tracts of the brain, so we speculated that the reduction of MBP had indeed an effect on myelin structure. However T2-weighted images contrast alterations could not be univocally attributed to myelin damages (Symms, Jager et al. 2004, Barkovich 2005, Tanaka, Iwasaki et al. 2012).

Consequently, we proceeded with complementary non-conventional MRI techniques, with focus on myelin and fibre bundles, namely MT and DTI. MT technique relies on the property of a tissue, e.g. myelin, to exchange magnetisation from its macromolecular proton pool to the protons of biological liquids and indirectly estimates the amount of water protons associated with macromolecules(Henkelman, Stanisz et al. 2001). MT is much more sensitive than conventional MRI in detecting ultrastructural changes in the brain tissue and several studies have showed a clear correlation between 
MT parameters and myelin content (Grossman, Gomori et al. 1994, Benedetti, Charil et al. 2006). The genotype-dependent reduction in Magnetisation Transfer Ratio (MTR), in Magnetisation Transfer (MT) saturation and the increase in T1 strongly suggested myelin alterations.

Surprisingly, DTI parameters were not affected by the reduction in MBP. DTI in the brain measures the diffusivity of water molecules along the axons, giving an estimation of both perpendicular and transversal water diffusion in respect to axons (Basser, Mattiello et al. 1994, Le Bihan, Mangin et al. 2001). In healthy axons, the water particles are restricted to one preferential direction (anisotropic movement), whereas a damaged axon allows a more disordered and unrestricted movement of water molecules (less anisotropic movement) (Basser, Mattiello et al. 1994, Le Bihan, Mangin et al. 2001). Intuitively, in case of myelin disruption the motion of water molecules would be less restricted (Stikov, Campbell et al. 2015).

Indeed, animal models of de-myelination show reduction in Fractional Anisotropy (FA) and an increase in radial diffusivity (RD), as indication of less organised motion of water molecules (Fatemi, Folsom et al. 2009). However, this is not observed in our model.

To understand the incongruities between MRI data we proceed with analysis of myelin ultrastructure of the corpus callosum of Mbp+/- vs. wild-type mice.

Our ultrastructural analysis of the rostral corpus callosum in senior mice suggests a trend in reduction of myelin thickness, especially in small calibre axons. This finding is in line with the studies on myelin development and on model of demyelination. Smallcalibre axons are normally myelinated after big-diameter axons (Remahl and Hildebrand 1982, Hildebrand, Remahl et al. 1993) and in mouse models of demyelination/hypo-myelination small-calibre axons are often the most affected ones (Umemori, Sato et al. 1994, Klugmann, Schwab et al. 1997, He, Dupree et al. 2007). One could speculate that, upon reduction of myelin component, the CNS might need to adapt and give priority axons subtypes over others.

It is worth mentioning that a bigger sample size and the analysis on 6-month old animals would be required to complement the EM data (note the significant inter individual variability); however, the current results support the MT readouts, suggesting mild alteration in $\mathrm{Mbp}+/-$ myelin structure.

Then, if myelin is really altered in Mbp+/-, how can we interpret such "inconsistency" between MT and DTI? In schizophrenia patients the abnormalities in MT parameters are 
generally accompanied by alteration in DTI readouts; however it is not uncommon to detect MTR reduction without DTI changes and vice-versa, indicating how these two methods are only partially overlapping in the detection of biological substrates (Kubicki, Park et al. 2005). One possible explanation would be that MT might be more sensitive than DTI to subtle changes that uniquely affect myelin (Kubicki, Park et al. 2005). Shiverer total knock-out mice miss both alleles for MBP and lack of compact myelin in the CNS; however they present an average of $10-15 \%$ reduction in FA compared to wild-type (Nair, Tanahashi et al. 2005, Tyszka, Readhead et al. 2006). We know from the literature that shiverer mice do not have major axonal alteration and it has been speculated that the anisotropy is partially preserved by such axonal integrity, even if myelin is not compacted (Song, Sun et al. 2002). Since our MRS data suggest preservation of neuronal function and density also in $\mathrm{Mbp}+/$ - corpus callosum, i.e. comparable N-Acetylaspartate (NAA) concentration between groups(Duarte, Do et al. 2014), one could speculate that Mbp+/- mice do not show any major difference in DTI because the axonal tracts are preserved and the myelin abnormalities are too subtle to be detected with this method.

Another explanatory model would be that the preservation of water directionality could be ensured by other glial cells surrounding the abnormally myelinated axons(Aung, Mar et al. 2013). A difference in the cell composition could also justify the major difference in contrast detected in T2-weighted images that could not be explained only by subtle alteration in myelin.

Our MRS data are more supportive of the second model. We detected a general increase in myo-Inositol in cortex and corpus callosum of Mbp+/- vs. wild-type. Myo-Inositol is a precursor in the phosphatidylinositol second messenger system and it is required for synthesis of membrane phospholipids (Kim, McGrath et al. 2005). Myo-Inositol is also an important osmolyte in glial cells and its increase is generally associated with gliosis (Taylor, Selvaraj et al. 2009). We indeed detected gliosis in the rostral corpus callosum of $\mathrm{Mbp}+/-$ mice, with a $30 \%$ increase in Iba1+ positive cells. We did not stain for Iba1+ cells in the cortex, but we expect a similar response also in this area.

Interestingly, increase in microglia and inflammation markers has also been reported in schizophrenia patients (Chew, Fusar-Poli et al. 2013, Fillman, Cloonan et al. 2013), even if not consistently (Chiappelli, Rowland et al. 2015). 
The biological meaning of this increase is still obscure. Microglia can secrete cytotoxic molecules and it is found to be activated in tissue of patients affected by neurodegenerative and white matter disorders (Peferoen, Kipp et al. 2014), but other studies also show that microglia is relevant for myelin synthesis, myelination and clearance of myelin debris (Hamilton and Rome 1994, Howe, Mayoral et al. 2006, Miron, Boyd et al. 2013). From our data it is not possible to draw definitive conclusion. The increase of microglia could serve as an homeostatic response to MBP reduction, to catch up with the subtle defect in myelination. In this frame, microglia could secrete anti-inflammatory cytokines and growth factors to promote myelination(Miron, Boyd et al. 2013). Alternatively, the increase in microglia could be a manifestation of an inflammatory response due to the abnormalities in myelin ultrastructure. In this case, microglia would secrete pro-inflammatory cytokines and reactive oxygen species (Miron, Boyd et al. 2013). Further studies are required to clarify the role of the increase in microglia in $\mathrm{Mbp}+/$ -

Myo-Inositol was not the only altered metabolites in the Mbp+/- brain. We also detected an increase in Taurine in both corpus callosum and cortex. Taurine is the second most abundant metabolite in the brain after glutamate(Bulley and Shen 2010) and its transporter is found both in astroglial cells and neurons, especially in glutamatergic neurons (Bulley and Shen 2010). Its role in the brain is still unclear, but it seems to work as osmolyte and neuroprotective agent, especially in case of glutamate-induced excitoxicity (Zwingmann, Leibfritz et al. 2003, Albrecht and Schousboe 2005, Wu and Prentice 2010). Taurine is 3-4 fold higher in neonates than in adults (Shivaraj, Marcy et al. 2012)and supplementation of taurine to ageing mice was reported to delay the agedependent cognitive decline (El Idrissi, Shen et al. 2013).

Higher level of taurine has been found in plasma and prefrontal cortex white matter of medicated schizophrenia patients compared with control (Prabakaran, Swatton et al. 2004, Samuelsson, Skogh et al. 2013).

The significantly higher level of taurine in $\mathrm{Mbp}+/$ - cortex could be related to the parallel increase of glutamate. On the one hand, it could be a compensatory mechanism to prevent axonal damage, caused by glutamate-related excitotoxic. On the other hand, the increase of glutamate could trigger exosome secretion from oligodendrocyte(Fruhbeis, Frohlich et al. 2013), with the aim of supporting the axon. In this view, the increase of 
taurine would be a concomitant mechanism to further support axons. However, we would need further confirmatory experiments to prove these points.

The other MRS changes in Mbp+/- mice are localised at cortical level. This is not too surprisingly, considering the peculiar regulation of MBP expression in $P F C$. On top of the increase of myo-Inositol and taurine, the cortex of $\mathrm{Mbp}+/-$ presented an increase in glutamate, GABA and total creatine.

GABA and glutamate are well-known neurotransmitter in the brain. Elevated cortical and white matter glutamate has been reported in early/acute psychosis and in nonmedicated patients (Chiappelli, Hong et al. 2015), moreover increase of GABA has also been detected in the prefrontal cortex of drug-naive patients (Port and Agarwal 2011). Glutamate excitotoxicity is supposed to be responsible for permanent brain damage (Port and Agarwal 2011), but from our data we cannot extrapolate whether we have excitotoxic process ongoing. On the other hand, it has been documented that the release of glutamate could also induce OPC proliferation and MBP translation (Barres and Raff 1993, Wake, Lee et al. 2011). As already mentioned, pools of OPCs survive in the adult brain as reservoir of precursor to produce oligodendrocytes. Considering the almost $70 \%$ reduction in MBP and the reduction trend in $\mathrm{CNP}$, the cortex of $\mathrm{Mbp}+/-$ could be in need of myelin and myelinating cells. The increase in glutamate could stimulate the proliferation of OPCs and MBP translation (Wake, Lee et al. 2011); the parallel increase in myo-Inositol (microglia) could promote differentiation (Miron, Boyd et al. 2013). Last but not least, glutamate increase could be a footprint of increased neural activity, which could promote myelination (Petersen and Monk 2015) or

The increase in total creatine is in line with both these speculations. In fact creatine is a shuttle of high-energy phosphate for ATP production that could be involved in both processes (Brosnan and Brosnan 2007). Further experiments are definitely required to explain the metabolic alteration in $\mathrm{Mbp}+/-$ mice.

Independently of the genotype, another interesting finding emerged from the MRI and MRS data. MT, MTR, FA are higher in "senior" vs. "mature" animals; moreover, total brain volume and NAA are unchanged and white matter volume in the corpus callosum is bigger in "senior" mice vs. "mature" mice. As mentioned, MTR and FA reduce in case of pathology, but also in case of physiological ageing; until adult midlife, white matter volume keeps increasing (Benedetti, Charil et al. 2006). On the other hand 
glutamate, GABA and taurine are significantly lower in "senior" animals compared to "mature" and a reduction of glutamate and GABA is generally detected upon ageing, as an indication of the reduction in synaptic functions, reduction of creatine (Duarte, Do et al. 2014).

Thus, this data suggest that the ageing process has most likely just started in "senior" animals and the first change comes from alteration of brain metabolism, specifically at neurotransmitter level.

The ultimate aim of my $\mathrm{PhD}$ research was then to explore the consequences of morphologic and metabolic alteration, induced by MBP reduction, on behavioural phenotypes.

The necessary prerequisite for the translational validity of this mouse model was to show that $\mathrm{Mbp}+/-$ mice did not present any gross basic functional alteration. Impairment in basic functions would have prevented reliable testing of other more complex behavioural domains.

Body weight, locomotion, swimming and basic perceptions (hearing, smell, sight and pain perception) were completely comparable in $\mathrm{Mbp}+/-$ vs. wild-type. This basic comparability between the two groups allowed me to proceed with higher function testing.

The alteration in the corpus callosum and the mild myelin abnormalities gave me a hint for setting up a test to evaluate fine motor functions and handedness in Mbp+/- vs. wildtype. It has been reported that mice missing the corpus callosum show altered paw preference (Ribeiro, Eales et al. 2013); moreover, in human, the inter-hemispheric connections, mediated by the corpus callosum, are crucial for timing and accuracy of motor tasks and motor control and they are important for bimanual and unimanual motor skills (Wahl, Lauterbach-Soon et al. 2007, Takeuchi, Oouchida et al. 2012). In parallel, since it has been reported that schizophrenia patients manifest subtle neurological symptoms (Walther and Strik 2012), I wandered if the subtle abnormality in myelin could be the biological base for fine motor function alteration.

We set up a test to measure these skills in mice, i.e. "grasping test". This test derives from the combination of paw preference test, first described by Collins in 1975 (Collins 1975), and the skilled reaching test, to quantify grasping abilities (Miklyaeva, Castaneda et al. 1994). However, I did not detect any genotype-related difference in 
grasping abilities or paw preference, suggesting that MBP reduction does not play a major role in this domain.

Schizophrenia patients show also cognitive impairment, social withdrawal, depression and anxiety (Ross, Margolis et al. 2006) and in mouse model these symptoms have been associated with reduction in myelin proteins and alteration in myelin (Liu, Dietz et al. 2012, Makinodan, Rosen et al. 2012). Consequently, I challenged the animals with several different cognitive and social tasks and I quantified the anxiety-like and depression-like behaviour. Unexpectedly, I did not detect any difference between the two groups, suggesting that MBP reduction does not have striking consequences on sociability and memory and it is unlikely to have a major effect on depression-like and anxiety-like symptoms in schizophrenia.

Schizophrenia patients are also characterised by sensorimotor gating deficits, meaning that they are not able to properly screen out irrelevant sensory stimuli from the environment (Braff and Light 2005, Kumari, Fannon et al. 2008). The sensorimotor gating impairment could be causative of sensory overload and cognitive fragmentation, typical of schizophrenia patients(Geyer 2006). Interestingly, this deficit has also been described in unaffected biological siblings of schizophrenia patients, pointing out a genetic contribution to the development of sensorimotor gating impairment (Kumari, Das et al. 2005). Both in human and in rodents, sensorimotor gating alteration has been correlated to cortical grey matter volume, myelin abnormalities and also to inflammatory processes (Fan, Goff et al. 2007, Kumari, Fannon et al. 2008, Takahashi, Sakurai et al. 2011). Thus, we wandered whether the reduction in MBP, and the consequent morphological and metabolic alteration accompanied by gliosis, led to alteration in prepulse inhibition (PPI) of acoustic startle response, a cross-species measure for sensorimotor gating. We found a reduction in PPI in $\mathrm{Mbp}+/-$ mice compared with wild-type, independently of the age.

PPI is considered to be mediated by the cortico-striato-pallido-thalamic circuitry in both mice and human (Kumari, Fannon et al. 2008, Ahmari, Risbrough et al. 2012) and it relies on several different signalling pathways.

One could speculate that mild abnormalities in myelin (especially callosal and cortical myelin) led to reduced synchronisation among brain regions. Neurotransmitter (GABA and glutamate) misbalances and gliosis could follow and induce the impairment in sensorimotor gating, via a general alteration of the neuronal network. 
The idea of a general "network disturbance disorder" more than a "single pathway disease" would also reconcile the different hypotheses of schizophrenia, which involve several different molecular pathway (Takahashi, Sakurai et al. 2011). Additionally to the PPI deficit, we also detect a reduction of acoustic startle response in "senior" $\mathrm{Mbp}+/$ - vs. wild-type mice. The primary circuitry of the pure acoustic startle response is located in the lower brainstem and it seems to be relatively simple when compared to the PPI (Koch 1999). Reduction in baseline startle reactivity has been associated with depressive episodes (Kaviani, Gray et al. 2004, Aaron T. Beck 2011). Whether Mbp+/mice show a reduced baseline startle as a first manifestation of depressive-like phenotype is not clear and it cannot be concluded from our data. Further analyses are required to better understand this behavioural readout.

In conclusion, our results suggest that the reduction of MBP could be partially causative of part of the myelin abnormalities detected in schizophrenia patients, affecting primarily the frontal region of the brain. The consequences of myelin alteration could have an impact on the synchronisation among various brain regions, leading to alteration in neurotransmitters, gliosis and network dysfunctions.

\section{TRANSLATIONAL VALIDITY OF THE MODEL AND OUTLOOK FOR FUTURE RESEARCH}

The mouse model we characterised was a first attempt to understand the role of MBP reduction (and MBP-related abnormalities in myelin) in schizophrenia. Specifically, we wanted to define which symptoms/sub-phenotypes could be attributed to the reduction of MBP over life. Table 6 summarises the data from Mbp+/- mice and compares them with the parallel finding in schizophrenia patients.

It is worth mentioning that this mouse model has two main limitations: (1) it is a conventional knock-out and (2) it allows exploring the consequences of MBP reduction, but it does not explain why such variation occurs in schizophrenia patients.

The first point leads to a major problem in relation to the construct validity of the model. We ensure a reduction in MBP expression via removal of one $M b p$ allele in the whole body since birth. 
However, the deletion of $M b p$ does not resemble the condition of schizophrenia patients, which have reduction in $M b p$ expression in the cortical areas and not $M b p$ mutation/deletion. To overcome this problem and to optimise the validity of the mouse model, one possibility would be to reduce MBP expression in cortical areas via silencing RNA technology. This would allow a specific spatiotemporal reduction of MBP expression that could be more informative for our purposes.

The second point is more complicated to approach, because the causes of schizophrenia are still unclear. Gene, environment and gene-environment interaction are all contributing to the development of the pathology, but how (and if) they specifically affect MBP expression is not known. However, despite its limitations, the Mbp+/model could be used to shed light on the causes of "myelin-related sub-phenotypes" in schizophrenia.

To study the role of environment per se on "myelin-related sub-phenotype" in schizophrenia, one possibility would be to expose wild-type animals to environmental stimuli, resembling known risk factors for schizophrenia in human, for instance cannabis consumption (Zammit, Allebeck et al. 2002).

Our $\mathrm{Mbp}+/-$ model could then come into play and could be used for comparative purposes. In this way, one could have a hint on which environmental conditions are likely to lead to MBP reduction and the consequent morphological, metabolic and behavioural alteration we detected in $\mathrm{Mbp}+/$ - mice.

To explore the effect of the gene-environment interaction one possibility would be to expose wild-type and $\mathrm{Mbp}+/$ - animals to environmental stimuli, that are known to alter myelination and to be potential risk factors for developing schizophrenia, for instance, social isolation (Hoffman 2007, Liu, Dietz et al. 2012, Makinodan, Rosen et al. 2012). Via these analyses, one could estimate the susceptibility to an environmental insult of animals that aregenetically predisposed to show schizophrenia-like sub-phenotypes $(\mathrm{Mbp}+/-$ mice $)$ in comparisonwith wild-type.

In summary, we showed that MBP reduction has indeed subtle, yet pathological, consequences and, these consequences resemble several phenotypes detected in schizophrenia patients. Consequently, being aware of the limitations of this model, in 
the context of our translational purposes $\mathrm{Mbp}+/-$ mouse couldbe used to study mild myelin alteration in schizophrenia. 


\section{DISCUSSION}

Table 6.Translational aspects of the $\mathrm{Mbp}+/-$ mouse model: comparison with findings in schizophrenia.

\begin{tabular}{|c|c|c|c|c|c|c|c|c|c|}
\hline & \multicolumn{2}{|c|}{3 Months } & \multicolumn{2}{|c|}{6 Months } & \multicolumn{2}{|c|}{18 Months } & \multicolumn{2}{|c|}{ Schizophrenia Patients } & \multirow[t]{2}{*}{ Reference } \\
\hline & Readout & Brain area & Readout & Brain area & Readout & Brain area & Changes & Where? & \\
\hline \multirow[t]{3}{*}{ Transcript } & $\downarrow \mathrm{Mbp}$ & $\begin{array}{c}\text { PFC, } \\
\text { Brainstem }\end{array}$ & $\downarrow M b p$ & PFC, Brainstem & $(\downarrow) M b p$ & $\begin{array}{c}\mathrm{PFC}, \\
\text { Brainstem }\end{array}$ & $\downarrow \mathrm{Mbp}$ & & $\begin{array}{l}\text { Parlapani et al., } \\
2009\end{array}$ \\
\hline & $=\mathrm{Plp} 1$ & $\begin{array}{c}\text { PFC, } \\
\text { Brainstem }\end{array}$ & $=P l p 1$ & PFC, Brainstem & $=P l p 1$ & $\begin{array}{c}\mathrm{PFC}, \\
\text { Brainstem }\end{array}$ & $\downarrow \mathrm{P} 1 \mathrm{p} 1$ & Prefrontal cortex & Tkachev et al., 2003 \\
\hline & $=\mathrm{Cnp}$ & $\begin{array}{c}\text { PFC, } \\
\text { Brainstem }\end{array}$ & $=C n p$ & PFC, Brainstem & $=C n p$ & $\begin{array}{c}\mathrm{PFC}, \\
\text { Brainstem }\end{array}$ & $\downarrow$ Cnp & $\begin{array}{c}\text { Dorsolateral } \\
\text { prefrontal cortex }\end{array}$ & Hakak et al., 2001 \\
\hline \multirow[t]{3}{*}{ Protein } & $\downarrow \mathrm{MBP}$ & $\begin{array}{c}\text { PFC, } \\
\text { Brainstem }\end{array}$ & $\downarrow \mathrm{MBP}$ & PFC, Brainstem & $\downarrow \downarrow \mathrm{MBP}$ & $\mathrm{PFC}$ & $\downarrow \mathrm{MBP}$ & Prefrontal cortex & Tkachev et al., 2003 \\
\hline & $\downarrow P L P$ & Brainstem & $=P l p 1$ & PFC, Brainstem & $=P l p 1$ & $\begin{array}{c}\mathrm{PFC}, \\
\text { Brainstem }\end{array}$ & $\downarrow$ PLP & & \\
\hline & $=\mathrm{Cnp}$ & $\begin{array}{c}\text { PFC, } \\
\text { Brainstem }\end{array}$ & $=C n p$ & PFC, Brainstem & $=C n p$ & $\begin{array}{c}\mathrm{PFC}, \\
\text { Brainstem }\end{array}$ & $\downarrow \mathrm{CNP}$ & $\begin{array}{l}\text { Anterior prefrontal } \\
\text { cortex }\end{array}$ & Flynn et al., 2003 \\
\hline Volumetry & NA & NA & $=$ & All & $=$ & All & $\begin{array}{c}\downarrow \text { Mean } \\
\text { Cerebral } \\
\text { Volume } \\
\uparrow \text { Ventricular } \\
\text { Volume }\end{array}$ & Overall & Konrad et al., 2007 \\
\hline $\begin{array}{l}\text { Convention } \\
\text { al MRI }\end{array}$ & NA & NA & $\begin{array}{c}\downarrow \text { white } \\
\text { matter } \\
\text { volume } \\
\downarrow \text { grey-white } \\
\text { matter } \\
\text { contrast }\end{array}$ & Corpus Callosum & $\begin{array}{c}\downarrow \text { white matter } \\
\text { volume } \downarrow \text { grey- } \\
\text { white matter } \\
\text { contrast }\end{array}$ & $\begin{array}{l}\text { Corpus } \\
\text { Callosum }\end{array}$ & $\begin{array}{c}\downarrow \text { white } \\
\text { matter } \\
\text { volume } \\
\downarrow \text { grey-white } \\
\text { matter } \\
\text { contrast }\end{array}$ & Corpus Callosum & Balevich et al., 2015 \\
\hline MT & NA & NA & $\begin{array}{c}\downarrow \mathrm{MTR}, \\
\downarrow \mathrm{MT}, \uparrow \mathrm{T} 1\end{array}$ & Corpus Callosum & $\downarrow \mathrm{MTR}, \downarrow \mathrm{MT}, \uparrow \mathrm{T} 1$ & $\begin{array}{l}\text { Corpus } \\
\text { Callosum }\end{array}$ & $\downarrow \mathrm{MTR}, \downarrow \mathrm{MT}$ & $\begin{array}{l}\text { Corpus callosum, } \\
\text { fornix.. }\end{array}$ & Kubicki et al., 2005; \\
\hline DTI & NA & NA & $=$ & - & $=$ & - & $\downarrow \mathrm{FA}$ & $\begin{array}{l}\text { Whole brain, Corpus } \\
\text { Callosum, }\end{array}$ & $\begin{array}{l}\text { Kubicki et al., 2005; } \\
\text { Kochunov et al., } \\
2013 \text {; Li et al., } 2013\end{array}$ \\
\hline \multirow{6}{*}{ MRS } & & & $\begin{array}{l}\uparrow \text { myo- } \\
\text { Inositol }\end{array}$ & Corpus Callosum & $\uparrow$ myo-Inositol & $\begin{array}{l}\text { Corpus } \\
\text { Callosum, } \\
\text { Cortex }\end{array}$ & $\begin{array}{l}\text { Abnormal } \\
\text { (not } \\
\text { consistent) }\end{array}$ & & $\begin{array}{l}\text { Deicken et al. 2000; } \\
\text { Kim et al. 2005; } \\
\text { Steen et al. } 2005\end{array}$ \\
\hline & & & $(\uparrow)$ Taurine & Cortex & $\uparrow$ Taurine & $\begin{array}{l}\text { Corpus } \\
\text { Callosum, } \\
\text { Cortex }\end{array}$ & $\begin{array}{l}\uparrow \text { Taurine/ } \\
=\text { Taurine }\end{array}$ & $\begin{array}{c}\text { Plasma/CSF } \\
\text { Cortex }\end{array}$ & $\begin{array}{c}\text { Bjerkenstedt et al., } \\
1985 ; \\
\text { Samuelsson et al, } \\
\text { 2013; Prabakaran et } \\
\text { al.. } 2004\end{array}$ \\
\hline & & NA & $=$ tCreatine & - & tCreatine & Cortex & $\begin{array}{l}\text { Abnormal } \\
\text { (not } \\
\text { consistent) }\end{array}$ & & $\begin{array}{l}\text { Deicken et al. } 2000 \\
\text { Kim et al. } 2005 \\
\text { Steen et al. } 2005\end{array}$ \\
\hline & & & $\uparrow \mathrm{GABA}$ & Cortex & $=\mathrm{GABA}$ & & $\downarrow$ GABA & Cortex & Lisman et al. 2008 \\
\hline & & & $\uparrow$ Glutamate & Cortex & $\uparrow$ Glutamate & Cortex & Abnormal & Cortex & $\begin{array}{l}\text { Marsman et al., } \\
2014\end{array}$ \\
\hline & & & $=\mathrm{NAA}$ & & $=\mathrm{NAA}$ & & $\downarrow N A A$ & Corpus callosum & Aydin et al., 2007 \\
\hline $\begin{array}{l}\text { Electron } \\
\text { Microscopy }\end{array}$ & & NA & & NA & $\begin{array}{c}\text { Reduction in } \\
\text { myelin thickness }\end{array}$ & $\begin{array}{l}\text { Rostral } \\
\text { corpus } \\
\text { callosum }\end{array}$ & $\begin{array}{l}\text { Axonal } \\
\text { Swelling, } \\
\text { Oligodendro } \\
\text { cyte } \\
\text { apoptosis, } \\
\text { gliosis }\end{array}$ & $\mathrm{PFC}$ & Uranova et al., 2007 \\
\hline Histology & & NA & & $\uparrow \mathrm{Micr}$ & roglia & & $\begin{array}{c}\text { Microglia } \\
\text { activation; } \\
\text { Inflammatio } \\
\text { n marker }\end{array}$ & $\begin{array}{l}\text { Gray matter; } \\
\text { PFC }\end{array}$ & $\begin{array}{l}\text { van Berckel et al., } \\
\quad 2008 \\
\text { Fillman et al., } 2013\end{array}$ \\
\hline Behaviour & \multicolumn{3}{|c|}{$\downarrow$ prepulse inhibition } & & $\begin{array}{r}\downarrow \text { prepulse inh } \\
\downarrow \text { baseline start }\end{array}$ & $\begin{array}{l}\text { libition } \\
\text { tle reflex }\end{array}$ & \multicolumn{2}{|c|}{ Sensorimotor gating disturbances } & Braff et al., 2001 \\
\hline
\end{tabular}

$\uparrow$ increase, $\downarrow$ decrease, = unchanged, NA Not available. References (from top to bottom of the table): (Deicken, Johnson et al. 2000, Braff, Geyer et al. 2001, Hakak, Walker et al. 2001, Flynn, Lang et al. 2003, Tkachev, Mimmack et al. 2003, Prabakaran, Swatton et al. 2004, Kim, McGrath et al. 2005, Kubicki, Park et al. 2005, Steen, Hamer et al. 2005, Aydin, Ucok et al. 2007, Uranova, Vostrikov et al. 2007, Konrad and Winterer 2008, Lisman, Coyle et al. 2008, van Berckel, Bossong et al. 2008, Parlapani, Schmitt et al. 2009, Fillman, Cloonan et al. 2013, Kochunov, Glahn et al. 2013, Samuelsson, Skogh et al. 2013, Li, Kale Edmiston et al. 2014, Marsman, Mandl et al. 2014, Balevich, Haznedar et al. 2015). 


\section{APPENDIX}

Table A. Myelin genes in schizophrenia.

Summary of the most replicated findings reported in the literature

\begin{tabular}{|c|c|c|c|c|c|}
\hline Gene & Brain Area & Technique & Results & p Value & Reference \\
\hline \multirow[t]{12}{*}{$\begin{array}{l}\text { MAL (Myelin and } \\
\text { Lymphocyte Protein) }\end{array}$} & \multicolumn{2}{|c|}{$\begin{array}{l}\text { Left hemispher Broadmann area Affimetrix HuGeneFL Chips } \\
\qquad 46\end{array}$} & -1.54 & 0.03 & Hakak et al., 2001 \\
\hline & \multicolumn{2}{|l|}{$\begin{array}{c}\text { Frontal, Cingulate, Temporal, } \\
\text { Parietal and Occipital Cortex, } \\
\text { Hippocampus, Caudate Nucleus } \\
\text { and Putamen }\end{array}$} & -1.98 & $<0.01$ & Katsel et al., 2005 \\
\hline & \multicolumn{2}{|c|}{$\begin{array}{l}\text { Left hemispher Broadmann area Affimetrix HuGeneFL Chips } \\
\qquad 47\end{array}$} & -1.87 & 0.0133 & Hakak et al., 2001 \\
\hline & Anterior Prefrontal Cortex & ELISA & $-33 \%$ & 0.05 & Flynn et al., 2003 \\
\hline & Broadmann area 9 & 2D-DIGE & $\begin{array}{l}-1-28 \text { to }- \\
1.63\end{array}$ & $\begin{array}{c}0.0024- \\
0.032\end{array}$ & $\begin{array}{l}\text { Prabakaran et al., } \\
2004\end{array}$ \\
\hline & \multicolumn{2}{|l|}{$\begin{array}{c}\text { Frontal, Cingulate, Temporal, } \\
\text { Parietal and Occipital Cortex, } \\
\text { Hippocampus, Caudate Nucleus } \\
\text { and Putamen }\end{array}$} & -1.95 & $<0.01$ & Katsel et al., 2005 \\
\hline & Hippocampus & TaqMan Real Time PCR & -1.86 & 0.005 & $\begin{array}{l}\text { Dracheva et al., } \\
2006\end{array}$ \\
\hline & Hippocampus & Western Blot & Decrease & 0.003 & $\begin{array}{l}\text { Dracheva et al., } \\
2006\end{array}$ \\
\hline & Anterior Cingulate Cortex & In Situ Hybridization & Decrease & $<0.04$ & $\begin{array}{l}\text { McCullumsmith } \\
\text { et al., } 2007\end{array}$ \\
\hline & Anterior Cingulate Gyrus & qPCR & -1.70 & 0.0004 & $\begin{array}{l}\text { Haroutunian et } \\
\quad \text { al., } 2007\end{array}$ \\
\hline & Anterior Cingulate Gyrus & HG U133A-B Array & -1.89 & 0.0033 & $\begin{array}{l}\text { Haroutunian et } \\
\quad \text { al., } 2007\end{array}$ \\
\hline & $\begin{array}{c}\text { Anteroventral and Mediodorsal } \\
\text { Thalamic Nuclei, Internal } \\
\text { Capsule and Putamen }\end{array}$ & qPCR & Decrease & 0.0035 & Barley et al., 2009 \\
\hline \multirow[t]{9}{*}{$\begin{array}{l}\text { MAG (Myelin-associated } \\
\text { Glycoprotein) }\end{array}$} & \multicolumn{2}{|c|}{$\begin{array}{l}\text { Left hemispher Broadmann area Affimetrix HuGeneFL Chips } \\
48\end{array}$} & -1.52 & 0.0024 & Hakak et al., 2001 \\
\hline & Broadmann area 9 & DDRT-PCR & -3.33 & 0.0001 & $\begin{array}{l}\text { Tkachev et al., } \\
2003\end{array}$ \\
\hline & Broadmann area 9 & Genome U133A Array & -3.00 & 0.0230 & $\begin{array}{l}\text { Tkachev et al., } \\
2003\end{array}$ \\
\hline & \multicolumn{2}{|l|}{$\begin{array}{c}\text { Frontal, Cingulate, Temporal, } \\
\text { Parietal and Occipital Cortex, } \\
\text { Hippocampus, Caudate Nucleus } \\
\text { and Putamen }\end{array}$} & -2.34 & $<0.01$ & Katsel et al., 2005 \\
\hline & Hippocampus & TaqMan Real Time PCR & -2.14 & 0.005 & $\begin{array}{l}\text { Dracheva et al., } \\
2006\end{array}$ \\
\hline & Anterior Cingulate Cortex & In Situ Hybridization & Decrease & $<0.04$ & $\begin{array}{l}\text { McCullumsmith } \\
\text { et et., } 2007\end{array}$ \\
\hline & Anterior Cingulate Gyrus & qPCR & -1.83 & 0.0005 & $\begin{array}{l}\text { Haroutunian et } \\
\quad \text { al., } 2007\end{array}$ \\
\hline & Anterior Cingulate Gyrus & HG U133A-B Array & -2.05 & 0.0450 & $\begin{array}{l}\text { Haroutunian et } \\
\quad \text { al., } 2007\end{array}$ \\
\hline & $\begin{array}{l}\text { Anteroventral and Mediodorsal } \\
\text { Thalamic Nuclei, Internal } \\
\text { Capsule and Putamen }\end{array}$ & qPCR & Decrease & 0.034 & Barley et al., 2009 \\
\hline
\end{tabular}




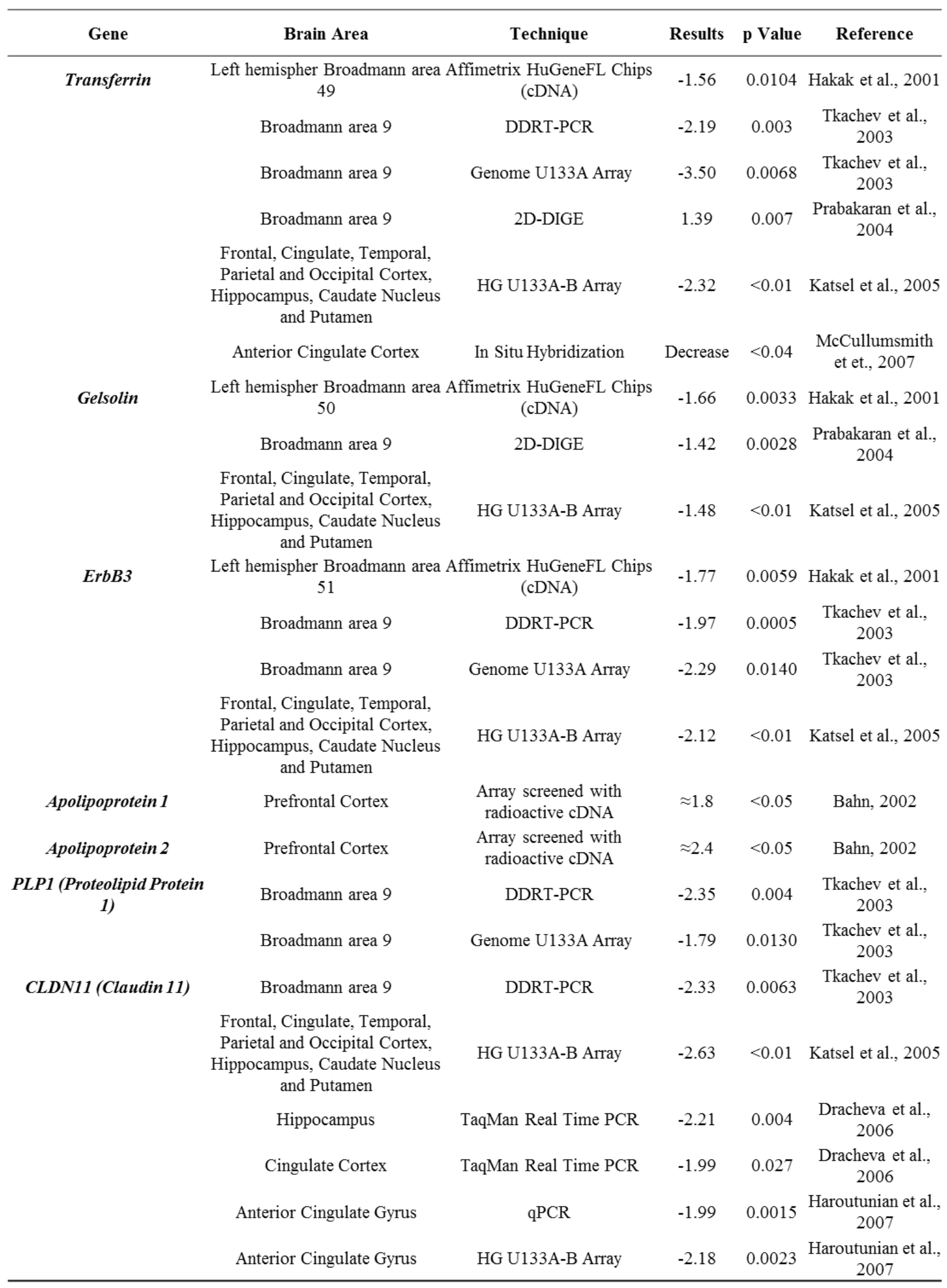

Table: part 2/3 


\begin{tabular}{|c|c|c|c|c|c|}
\hline Gene & Brain Area & Technique & Results & p Value & Reference \\
\hline \multirow[t]{4}{*}{$\begin{array}{l}\text { MOG (Myelin } \\
\text { Oligodendrocyte } \\
\text { Glycoprotein) }\end{array}$} & Broadmann area 9 & DDRT-PCR & -2.58 & 0.0039 & $\begin{array}{l}\text { Tkachev et al., } \\
2003\end{array}$ \\
\hline & Broadmann area 9 & Genome U133A Array & -2.91 & 0.0070 & $\begin{array}{l}\text { Tkachev et al., } \\
2003\end{array}$ \\
\hline & $\begin{array}{c}\text { Frontal, Cingulate, Temporal, } \\
\text { Parietal and Occipital Cortex, } \\
\text { Hippocampus, Caudate Nucleus } \\
\text { and Putamen }\end{array}$ & HG U133A-B Array & -2.05 & $<0.01$ & Katsel et al., 2005 \\
\hline & $\begin{array}{l}\text { Anteroventral and Mediodorsal } \\
\text { Thalamic Nuclei, Internal } \\
\text { Capsule and Putamen }\end{array}$ & $\mathrm{qPCR}$ & Decrease & 0.043 & Barley et al., 2009 \\
\hline \multirow[t]{2}{*}{$\begin{array}{c}\text { MPZL1 (Myelin Protein } \\
\text { Zero 1) }\end{array}$} & Broadmann area 9 & DDRT-PCR & 1.29 & 0.0263 & $\begin{array}{l}\text { Tkachev et al., } \\
2003\end{array}$ \\
\hline & Broadmann area 9 & Genome U133A Array & 1.20 & 0.0120 & $\begin{array}{l}\text { Tkachev et al., } \\
2003\end{array}$ \\
\hline OLIG 1 & Broadmann area 9 & DDRT-PCR & -1.74 & 0.0190 & $\begin{array}{c}\text { Tkachev et al., } \\
2003\end{array}$ \\
\hline \multirow[t]{3}{*}{ OLIG2 } & Broadmann area 9 & DDRT-PCR & -1.65 & 0.0027 & $\begin{array}{c}\text { Tkachev et al., } \\
2003\end{array}$ \\
\hline & Broadmann area 9 & Genome U133A Array & -2.41 & 0.0010 & $\begin{array}{c}\text { Tkachev et al., } \\
2003\end{array}$ \\
\hline & $\begin{array}{c}\text { Frontal, Cingulate, Temporal, } \\
\text { Parietal and Occipital Cortex, } \\
\text { Hippocampus, Caudate Nucleus } \\
\text { and Putamen }\end{array}$ & HG U133A-B Array & -1.68 & $<0.01$ & Katsel et al., 2005 \\
\hline \multirow[t]{6}{*}{ SOX10 } & Broadmann area 9 & DDRT-PCR & -1.99 & 0.0029 & $\begin{array}{l}\text { Tkachev et al., } \\
2003\end{array}$ \\
\hline & Broadmann area 9 & Genome U133A Array & -1.88 & 0.0070 & $\begin{array}{c}\text { Tkachev et al., } \\
2003\end{array}$ \\
\hline & $\begin{array}{c}\text { Frontal, Cingulate, Temporal, } \\
\text { Parietal and Occipital Cortex, } \\
\text { Hippocampus, Caudate Nucleus } \\
\text { and Putamen }\end{array}$ & HG U133A-B Array & -1.74 & $<0.01$ & Katsel et al., 2005 \\
\hline & Hippocampus & TaqMan Real Time PCR & -1.84 & 0.003 & $\begin{array}{l}\text { Dracheva et al., } \\
2006\end{array}$ \\
\hline & Cingulate Cortex & TaqMan Real Time PCR & -1.93 & 0.014 & $\begin{array}{l}\text { Dracheva et al., } \\
2006\end{array}$ \\
\hline & Anterior Cingulate Gyrus & $\mathrm{qPCR}$ & -1.93 & 0.0003 & \\
\hline \multirow[t]{2}{*}{$\begin{array}{c}C A L G \\
\text { (galactosylceramidase) }\end{array}$} & Broadmann area 9 & Genome U133A Array & -1.24 & 0.0480 & $\begin{array}{l}\text { Tkachev et al., } \\
2003\end{array}$ \\
\hline & $\begin{array}{l}\text { Anteroventral and Mediodorsal } \\
\text { Thalamic Nuclei, Internal } \\
\text { Capsule and Putamen }\end{array}$ & $\mathrm{qPCR}$ & Decrease & 0.031 & Barley et al., 2009 \\
\hline \multirow[t]{3}{*}{$\begin{array}{c}\text { MOBP (myelin-associated } \\
\text { oligodendrocyte basic } \\
\text { protein) }\end{array}$} & Broadmann area 9 & Genome U133A Array & -2.27 & 0.0400 & $\begin{array}{l}\text { Tkachev et al., } \\
2003\end{array}$ \\
\hline & $\begin{array}{c}\text { Frontal, Cingulate, Temporal, } \\
\text { Parietal and Occipital Cortex, } \\
\text { Hippocampus, Caudate Nucleus } \\
\text { and Putamen }\end{array}$ & HG U133A-B Array & -2.28 & $<0.01$ & Katsel et al., 2005 \\
\hline & Anterior Cingulate Cortex & In Situ Hybridization & Decrease & $<0.03$ & $\begin{array}{l}\text { McCullumsmith } \\
\text { et et., } 2007\end{array}$ \\
\hline$N G 2$ & $\begin{array}{l}\text { Anteroventral and Mediodorsal } \\
\text { Thalamic Nuclei, Internal } \\
\text { Capsule and Putamen }\end{array}$ & $\mathrm{qPCR}$ & Increase & 0.004 & Barley et al., 2009 \\
\hline
\end{tabular}

Table: part 3/3; Reference (from top to bottom of the entire table):(Hakak, Walker et al. 2001, Flynn, Lang et al. 2003, Prabakaran, Swatton et al. 2004, Katsel, Davis et al. 2005, Dracheva, Davis et al. 2006, Haroutunian, Katsel et al. 2007, McCullumsmith, Gupta et al. 2007, Barley, Dracheva et al. 2009) 


\section{LIST OF ABBREVIATIONS}

\begin{tabular}{|c|c|}
\hline Abbreviation & Extended name \\
\hline $\mathrm{AD}$ & Axial Diffusivity \\
\hline $\mathrm{ADC}$ & Apparent Diffusion Coefficient \\
\hline $\mathrm{BBB}$ & Blood Brain Barrier \\
\hline CALG & Galactosylceramidase \\
\hline CLDN11 & Claudin 11 \\
\hline $\mathrm{CNP}$ & $2^{\prime}: 3^{\prime}$-Cyclic nucleotide 3'-phosphodiesterase \\
\hline $\mathrm{CNS}$ & Central Nervous System \\
\hline DAPI & 4',6-Diamidino-2-phenylindole \\
\hline EM & Electron Microscopy \\
\hline FA & Fractional Anisotropy \\
\hline GABA & gamma-Aminobutyric acid \\
\hline golli & genes of oligodendrocytes lineage \\
\hline GWAS & Genome-Wide Association Studies \\
\hline hnRNP & Heterogeneous nuclear Ribonucleoprotein \\
\hline IBA1 & Ionized calcium-Binding Adapter molecule 1 \\
\hline kif1b & Kinesin Family Member $1 b$ \\
\hline MAG & Myelin Associated Glycoprotein \\
\hline MAG & Myelin Associated Glycoprotein \\
\hline MBP & Myelin Basic Protein \\
\hline MOBP & $\begin{array}{l}\text { Myelin-associated Oligodendrocyte basic } \\
\text { protein }\end{array}$ \\
\hline MOG & Myelin Oligodendrocyte Glicoprotein \\
\hline MPZL1 & Myelin Protein Zero 1 \\
\hline MRI & Magnetic Resonance Imaging \\
\hline mRNA & Messenger RNA \\
\hline MRS & Magnetic Resonance Spectroscopy \\
\hline MT sat & Magnetization saturation \\
\hline MTR & Magnetisation Transfer Ratio \\
\hline NAA & N-Acetylaspartate \\
\hline NG2 & Neural/Glial antigen 2 \\
\hline
\end{tabular}


Olig1 Oligodendrocyte Transcription Factor 1

Olig2 Oligodendrocyte Transcription Factor 2

OPCs Oligodendrocyte Precursor Cells

OR

Odds Ratio

P21-P35

P23

Post-natal day 21-Post-natal day 35

PCR

Post-natal day 23

PDGFR-

aplha

PFC

Polymerase Chain Reaction

platelet-derived growth factor receptor -alpha

PIP2

Prefrontal Cortex

PIP3

Phosphatidylinositol 4,5-bisphosphate

PLP

phosphatidylinositol-(3,4,5,)-triphosphate

PPI

Proteolipid protein

PTM

QK1

RD

RLR

RNA

Sirt2

Pre-pulse inhibition of startle response

Post-translational Modification

Protein Quacking 1

Radial Diffusivity

RNA Localisation Region

Ribonucleic acid

SOX10

Sirtuin 2

TE

Sex Determining Region Y)-Box 10

TR

Echo Time

VOI

Repetition Time

Volume Of Interest 


\section{REFERENCES}

Aaron T. Beck, N. A. R., Neal Stolar, and Paul Grant (2011). "Schizofrenia, teoria cognitiva, ricerca e terapia " Casa Editrice Astrolabio (chapter 1).

Aggarwal, S., L. Yurlova and M. Simons (2011). "Central nervous system myelin: structure, synthesis and assembly." Trends Cell Biol21(10): 585-593.

Aggarwal, S., L. Yurlova, N. Snaidero, C. Reetz, S. Frey, J. Zimmermann, G. Pahler, A. Janshoff, J. Friedrichs, D. J. Muller, C. Goebel and M. Simons (2011). "A size barrier limits protein diffusion at the cell surface to generate lipid-rich myelin-membrane sheets." Dev Cell21(3): 445-456.

Ahmari, S. E., V. B. Risbrough, M. A. Geyer and H. B. Simpson (2012). "Impaired sensorimotor gating in unmedicated adults with obsessive-compulsive disorder." Neuropsychopharmacology37(5): 1216-1223.

Ainger, K., D. Avossa, A. S. Diana, C. Barry, E. Barbarese and J. H. Carson (1997). "Transport and localization elements in myelin basic protein mRNA." J Cell Biol138(5): 1077-1087.

Albrecht, J. and A. Schousboe (2005). "Taurine interaction with neurotransmitter receptors in the CNS: an update." Neurochem Res30(12): 1615-1621.

Allinquant, B., S. M. Staugaitis, D. D'Urso and D. R. Colman (1991). "The ectopic expression of myelin basic protein isoforms in Shiverer oligodendrocytes: implications for myelinogenesis." J Cell Biol113(2): 393-403.

Ansari, K. A. and J. Loch (1975). "Decreased myelin basic protein content of the aged human brain." Neurology25(11): 1045-1050.

Aung, W. Y., S. Mar and T. L. S. Benzinger (2013). "Diffusion tensor MRI as a biomarker in axonal and myelin damage." Imaging in medicine 5(5): 427-440.

Ayalew, M., H. Le-Niculescu, D. F. Levey, N. Jain, B. Changala, S. D. Patel, E. Winiger, A. Breier, A. Shekhar, R. Amdur, D. Koller, J. I. Nurnberger, A. Corvin, M. Geyer, M. T. Tsuang, D. Salomon, N. J. Schork, A. H. Fanous, M. C. O'Donovan and A. B. Niculescu (2012). "Convergent functional genomics of schizophrenia: from comprehensive understanding to genetic risk prediction." Mol Psychiatry17(9): 887905.

Aydin, K., A. Ucok and S. Cakir (2007). "Quantitative proton MR spectroscopy findings in the corpus callosum of patients with schizophrenia suggest callosal disconnection." AJNR Am J Neuroradiol28(10): 1968-1974.

Balevich, E. C., M. M. Haznedar, E. Wang, R. E. Newmark, R. Bloom, J. S. Schneiderman, J. Aronowitz, C. Y. Tang, K. W. Chu, W. Byne, M. S. Buchsbaum and E. A. Hazlett (2015). "Corpus callosum size and diffusion tensor anisotropy in adolescents and adults with schizophrenia." Psychiatry Res231(3): 244-251. 
Barbarese, E., M. L. Nielson and J. H. Carson (1983). "The effect of the shiverer mutation on myelin basic protein expression in homozygous and heterozygous mouse brain." J Neurochem40(6): 1680-1686.

Barkovich, A. J. (2005). "Magnetic resonance techniques in the assessment of myelin and myelination." J Inherit Metab Dis 28(3): 311-343.

Barley, K., S. Dracheva and W. Byne (2009). "Subcortical oligodendrocyte- and astrocyte-associated gene expression in subjects with schizophrenia, major depression and bipolar disorder." Schizophr Res112(1-3): 54-64.

Baron, W. and D. Hoekstra (2010). "On the biogenesis of myelin membranes: sorting, trafficking and cell polarity." FEBS Lett584(9): 1760-1770.

Barrera, K., P. Chu, J. Abramowitz, R. Steger, R. L. Ramos and J. C. Brumberg (2013). "Organization of myelin in the mouse somatosensory barrel cortex and the effects of sensory deprivation." Dev Neurobiol73(4): 297-314.

Barres, B. A. and M. C. Raff (1993). "Proliferation of oligodendrocyte precursor cells depends on electrical activity in axons." Nature361(6409): 258-260.

Basser, P. J., J. Mattiello and D. LeBihan (1994). "MR diffusion tensor spectroscopy and imaging." Biophys J66(1): 259-267.

Baumann, N. and D. Pham-Dinh (2001). "Biology of oligodendrocyte and myelin in the mammalian central nervous system." Physiol Rev81(2): 871-927.

Bechler, M. E. and C. ffrench-Constant (2014). "Neuroscience. A new wrap for neuronal activity?" Science344(6183): 480-481.

Bellesi, M., M. Pfister-Genskow, S. Maret, S. Keles, G. Tononi and C. Cirelli (2013). "Effects of sleep and wake on oligodendrocytes and their precursors." J Neurosci33(36): 14288-14300.

Benedetti, B., A. Charil, M. Rovaris, E. Judica, P. Valsasina, M. P. Sormani and M. Filippi (2006). "Influence of aging on brain gray and white matter changes assessed by conventional, MT, and DT MRI." Neurology66(4): 535-539.

Bercury, K. K. and W. B. Macklin (2015). "Dynamics and mechanisms of CNS myelination." Dev Cell32(4): 447-458.

Bevins, R. A. and J. Besheer (2006). "Object recognition in rats and mice: a one-trial non-matching-to-sample learning task to study 'recognition memory'." Nat Protoc1(3): $1306-1311$.

Boggs, J. M. (2006). "Myelin basic protein: a multifunctional protein." Cell Mol Life Sci63(17): 1945-1961.

Boggs, J. M., G. Rangaraj, Y. M. Heng, Y. Liu and G. Harauz (2011). "Myelin basic protein binds microtubules to a membrane surface and to actin filaments in vitro: effect of phosphorylation and deimination." Biochim Biophys Acta1808(3): 761-773. 
Boiko, T. and B. Winckler (2006). "Myelin under construction -- teamwork required." $\underline{\mathbf{J}}$ Cell Biol172(6): 799-801.

Braff, D., C. Stone, E. Callaway, M. Geyer, I. Glick and L. Bali (1978). "Prestimulus Effects on Human Startle Reflex in Normals and Schizophrenics." Psychophysiology15(4): 339-343.

Braff, D. L., M. A. Geyer and N. R. Swerdlow (2001). "Human studies of prepulse inhibition of startle: normal subjects, patient groups, and pharmacological studies." Psychopharmacology (Berl) 156(2-3): 234-258.

Braff, D. L. and G. A. Light (2005). "The use of neurophysiological endophenotypes to understand the genetic basis of schizophrenia." Dialogues Clin Neurosci7(2): 125-135.

Brosnan, J. T. and M. E. Brosnan (2007). "Creatine: endogenous metabolite, dietary, and therapeutic supplement." Annu Rev Nutr27: 241-261.

Buckley, P. F. (2005). "Neuroimaging of schizophrenia: structural abnormalities and pathophysiological implications." Neuropsychiatric Disease and Treatment1(3): 193204.

Bulley, S. and W. Shen (2010). "Reciprocal regulation between taurine and glutamate response via $\mathrm{Ca} 2+-d e p e n d e n t$ pathways in retinal third-order neurons." J Biomed Sci17 Suppl 1: S5.

Bunge, M. B., R. P. Bunge and H. Ris (1961). "Ultrastructural study of remyelination in an experimental lesion in adult cat spinal cord." J Biophys Biochem Cytol10: 67-94.

Camara, J., Z. Wang, C. Nunes-Fonseca, H. C. Friedman, M. Grove, D. L. Sherman, N. H. Komiyama, S. G. Grant, P. J. Brophy, A. Peterson and C. ffrench-Constant (2009). "Integrin-mediated axoglial interactions initiate myelination in the central nervous system." J Cell Biol185(4): 699-712.

Cammer, W. (1982). "Partial deficiencies in the myelin proteins of developing mice heterozygous for the shiverer mutation." Developmental Genetics3(2): 155-163.

Chambers, J. S. and N. I. Perrone-Bizzozero (2004). "Altered myelination of the hippocampal formation in subjects with schizophrenia and bipolar disorder." Neurochem Res29(12): 2293-2302.

Chernoff, G. F. (1981). "Shiverer: an autosomal recessive mutant mouse with myelin deficiency." J Hered72(2): 128.

Chetty, S., A. R. Friedman, K. Taravosh-Lahn, E. D. Kirby, C. Mirescu, F. Guo, D. Krupik, A. Nicholas, A. C. Geraghty, A. Krishnamurthy, M. K. Tsai, D. Covarrubias, A. T. Wong, D. D. Francis, R. M. Sapolsky, T. D. Palmer, D. Pleasure and D. Kaufer (2014). "Stress and glucocorticoids promote oligodendrogenesis in the adult hippocampus." Mol Psychiatry19(12): 1275-1283.

Chew, L. J., P. Fusar-Poli and T. Schmitz (2013). "Oligodendroglial alterations and the role of microglia in white matter injury: relevance to schizophrenia." Dev Neurosci35(2-3): 102-129. 
Chiappelli, J., L. E. Hong, S. A. Wijtenburg, X. Du, F. Gaston, P. Kochunov and L. M. Rowland (2015). "Alterations in frontal white matter neurochemistry and microstructure in schizophrenia: implications for neuroinflammation." Transl Psychiatry5: e548.

Chiappelli, J., L. M. Rowland, S. A. Wijtenburg, F. Muellerklein, M. Tagamets, R. P. McMahon, F. Gaston, P. Kochunov and L. E. Hong (2015). "Evaluation of Myo-Inositol as a Potential Biomarker for Depression in Schizophrenia." Neuropsychopharmacology40(9): 2157-2164.

Chong, S. Y. and J. R. Chan (2010). "Tapping into the glial reservoir: cells committed to remaining uncommitted." J Cell Biol188(3): 305-312.

Chrast, R., G. Saher, K. A. Nave and M. H. Verheijen (2011). "Lipid metabolism in myelinating glial cells: lessons from human inherited disorders and mouse models." $\underline{\mathrm{J}}$ Lipid Res52(3): 419-434.

Collins, R. L. (1975). "When left-handed mice live in right-handed worlds." Science 187(4172): 181-184.

Comfort, A. (1959). "Natural Aging and the Effects of Radiation." Radiation Research Supplement1: 216-234.

Consortium, T. S. P. G.-W. A. S. G. (2011). "Genome-wide association study identifies five new schizophrenia loci." Nat Genet43(10): 969-976.

Corrigan, P. W. and A. C. Watson (2002). "Understanding the impact of stigma on people with mental illness." World Psychiatry1(1): 16-20.

Dai, J., K. K. Bercury, J. T. Ahrendsen and W. B. Macklin (2015). "Olig1 function is required for oligodendrocyte differentiation in the mouse brain." J Neurosci35(10): 4386-4402.

Deber, C. M. and S. J. Reynolds (1991). "Central nervous system myelin: structure, function, and pathology." Clin Biochem24(2): 113-134.

Deicken, R. F., C. Johnson and M. Pegues (2000). "Proton magnetic resonance spectroscopy of the human brain in schizophrenia." Rev Neurosci11(2-3): 147-158.

Delassalle, A., B. Zalc, F. Lachapelle, M. Raoul, P. Collier and C. Jacque (1981). "Regional distribution of myelin basic protein in the central nervous system of quaking, jimpy, and normal mice during development and aging." J Neurosci Res6(3): 303-313.

Demerens, C., B. Stankoff, M. Logak, P. Anglade, B. Allinquant, F. Couraud, B. Zalc and C. Lubetzki (1996). "Induction of myelination in the central nervous system by electrical activity." Proc Natl Acad Sci U S A93(18): 9887-9892.

DeVito, L. M., C. Lykken, B. R. Kanter and H. Eichenbaum (2010). "Prefrontal cortex: role in acquisition of overlapping associations and transitive inference." Learn Mem17(3): 161-167.

Dracheva, S., K. L. Davis, B. Chin, D. A. Woo, J. Schmeidler and V. Haroutunian (2006). "Myelin-associated mRNA and protein expression deficits in the anterior 
cingulate cortex and hippocampus in elderly schizophrenia patients." Neurobiol Dis21(3): 531-540.

Duarte, J. M., K. Q. Do and R. Gruetter (2014). "Longitudinal neurochemical modifications in the aging mouse brain measured in vivo by $1 \mathrm{H}$ magnetic resonance spectroscopy." Neurobiol Aging35(7): 1660-1668.

El Idrissi, A., C. H. Shen and J. L'Amoreaux W (2013). "Neuroprotective role of taurine during aging." Amino Acids45(4): 735-750.

Elmore, M. R., A. R. Najafi, M. A. Koike, N. N. Dagher, E. E. Spangenberg, R. A. Rice, M. Kitazawa, B. Matusow, H. Nguyen, B. L. West and K. N. Green (2014). "Colony-stimulating factor 1 receptor signaling is necessary for microglia viability, unmasking a microglia progenitor cell in the adult brain." Neuron82(2): 380-397.

Fan, X., D. C. Goff and D. C. Henderson (2007). "Inflammation and schizophrenia." Expert Rev Neurother7(7): 789-796.

Fatemi, S. H., T. D. Folsom, T. J. Reutiman, D. Abu-Odeh, S. Mori, H. Huang and K. Oishi (2009). "Abnormal expression of myelination genes and alterations in white matter fractional anisotropy following prenatal viral influenza infection at E16 in mice." Schizophr Res112(1-3): 46-53.

Fields, R. D. (2005). "Myelination: an overlooked mechanism of synaptic plasticity?" Neuroscientist11(6): 528-531.

Fillman, S. G., N. Cloonan, L. C. Miller and C. S. Weickert (2013). "Markers of inflammation in the prefrontal cortex of individuals with schizophrenia." Mol Psychiatry18(2): 133.

Fitzner, D., A. Schneider, A. Kippert, W. Mobius, K. I. Willig, S. W. Hell, G. Bunt, K. Gaus and M. Simons (2006). "Myelin basic protein-dependent plasma membrane reorganization in the formation of myelin." EMBO J25(21): 5037-5048.

Flynn, S. W., D. J. Lang, A. L. Mackay, V. Goghari, I. M. Vavasour, K. P. Whittall, G. N. Smith, V. Arango, J. J. Mann, A. J. Dwork, P. Falkai and W. G. Honer (2003). "Abnormalities of myelination in schizophrenia detected in vivo with MRI, and postmortem with analysis of oligodendrocyte proteins." Mol Psychiatry8(9): 811-820.

Foran, D. R. and A. C. Peterson (1992). "Myelin acquisition in the central nervous system of the mouse revealed by an MBP-Lac Z transgene." J Neurosci12(12): 48904897.

Freeman, M. R. and D. H. Rowitch (2013). "Evolving concepts of gliogenesis: a look way back and ahead to the next 25 years." Neuron80(3): 613-623.

Fruhbeis, C., D. Frohlich, W. P. Kuo and E. M. Kramer-Albers (2013). "Extracellular vesicles as mediators of neuron-glia communication." Front Cell Neurosci7: 182.

Fulton, D., P. M. Paez and A. T. Campagnoni (2010). "The multiple roles of myelin

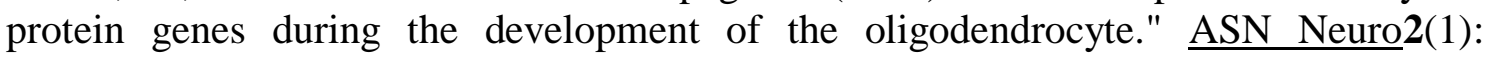
e00027. 
Funfschilling, U., L. M. Supplie, D. Mahad, S. Boretius, A. S. Saab, J. Edgar, B. G. Brinkmann, C. M. Kassmann, I. D. Tzvetanova, W. Mobius, F. Diaz, D. Meijer, U. Suter, B. Hamprecht, M. W. Sereda, C. T. Moraes, J. Frahm, S. Goebbels and K. A. Nave (2012). "Glycolytic oligodendrocytes maintain myelin and long-term axonal integrity." Nature485(7399): 517-521.

Geyer, M. A. (2006). "The family of sensorimotor gating disorders: comorbidities or diagnostic overlaps?" Neurotox Res10(3-4): 211-220.

Gibson, E. M., D. Purger, C. W. Mount, A. K. Goldstein, G. L. Lin, L. S. Wood, I. Inema, S. E. Miller, G. Bieri, J. B. Zuchero, B. A. Barres, P. J. Woo, H. Vogel and M. Monje (2014). "Neuronal activity promotes oligodendrogenesis and adaptive myelination in the mammalian brain." Science344(6183): 1252304.

Gottesman, II and T. D. Gould (2003). "The endophenotype concept in psychiatry: etymology and strategic intentions." Am J Psychiatry160(4): 636-645.

Goudriaan, A., C. de Leeuw, S. Ripke, C. M. Hultman, P. Sklar, P. F. Sullivan, A. B. Smit, D. Posthuma and M. H. Verheijen (2014). "Specific glial functions contribute to schizophrenia susceptibility." Schizophr Bull40(4): 925-935.

Grossman, R. I., J. M. Gomori, K. N. Ramer, F. J. Lexa and M. D. Schnall (1994). "Magnetization transfer: theory and clinical applications in neuroradiology." Radiographics 14(2): 279-290.

Gudz, T. I., H. Komuro and W. B. Macklin (2006). "Glutamate stimulates oligodendrocyte progenitor migration mediated via an alphav integrin/myelin proteolipid protein complex." J Neurosci26(9): 2458-2466.

Gur, R. E. and R. C. Gur (2010). "Functional magnetic resonance imaging in schizophrenia." Dialogues in Clinical Neuroscience 12(3): 333-343.

Gur, R. E., M. S. Keshavan and S. M. Lawrie (2007). "Deconstructing psychosis with human brain imaging." Schizophr Bull33(4): 921-931.

Hagemeyer, N., S. Goebbels, S. Papiol, A. Kastner, S. Hofer, M. Begemann, U. C. Gerwig, S. Boretius, G. L. Wieser, A. Ronnenberg, A. Gurvich, S. H. Heckers, J. Frahm, K. A. Nave and H. Ehrenreich (2012). "A myelin gene causative of a catatoniadepression syndrome upon aging." EMBO Mol Med4(6): 528-539.

Hakak, Y., J. R. Walker, C. Li, W. H. Wong, K. L. Davis, J. D. Buxbaum, V. Haroutunian and A. A. Fienberg (2001). "Genome-wide expression analysis reveals dysregulation of myelination-related genes in chronic schizophrenia." Proc Natl Acad Sci U S A98(8): 4746-4751.

Hall, J., S. Trent, K. L. Thomas, M. C. O'Donovan and M. J. Owen (2015). "Genetic risk for schizophrenia: convergence on synaptic pathways involved in plasticity." Biol Psychiatry77(1): 52-58.

Hamilton, S. P. and L. H. Rome (1994). "Stimulation of in vitro myelin synthesis by microglia." Glia11(4): 326-335. 
Harauz, G., V. Ladizhansky and J. M. Boggs (2009). "Structural polymorphism and multifunctionality of myelin basic protein." Biochemistry48(34): 8094-8104.

Haroutunian, V., P. Katsel, S. Dracheva, D. G. Stewart and K. L. Davis (2007). "Variations in oligodendrocyte-related gene expression across multiple cortical regions: implications for the pathophysiology of schizophrenia." Int $\mathrm{J}$ Neuropsychopharmacol10(4): 565-573.

Haroutunian, V., P. Katsel, P. Roussos, K. L. Davis, L. L. Altshuler and G. Bartzokis (2014). "Myelination, oligodendrocytes, and serious mental illness." Glia62(11): 18561877.

Hartline, D. K. and D. R. Colman (2007). "Rapid conduction and the evolution of giant axons and myelinated fibers." Curr Biol17(1): R29-35.

He, Y., J. Dupree, J. Wang, J. Sandoval, J. Li, H. Liu, Y. Shi, K. A. Nave and P. Casaccia-Bonnefil (2007). "The transcription factor Yin Yang 1 is essential for oligodendrocyte progenitor differentiation." Neuron55(2): 217-230.

Henkelman, R. M., G. J. Stanisz and S. J. Graham (2001). "Magnetization transfer in MRI: a review." NMR Biomed14(2): 57-64.

Hildebrand, C., S. Remahl, H. Persson and C. Bjartmar (1993). "Myelinated nerve fibres in the CNS." Prog Neurobiol40(3): 319-384.

Hines, J. H., A. M. Ravanelli, R. Schwindt, E. K. Scott and B. Appel (2015). "Neuronal activity biases axon selection for myelination in vivo." Nat Neurosci18(5): 683-689.

Hoffman, R. E. (2007). "A social deafferentation hypothesis for induction of active schizophrenia." Schizophr Bull33(5): 1066-1070.

Hoistad, M., D. Segal, N. Takahashi, T. Sakurai, J. D. Buxbaum and P. R. Hof (2009). "Linking white and grey matter in schizophrenia: oligodendrocyte and neuron pathology in the prefrontal cortex." Front Neuroanat3: 9.

Holleran, L., M. Ahmed, H. Anderson-Schmidt, J. McFarland, L. Emsell, A. Leemans, C. Scanlon, P. Dockery, P. McCarthy, G. J. Barker, C. McDonald and D. M. Cannon (2014). "Altered interhemispheric and temporal lobe white matter microstructural organization in severe chronic schizophrenia." Neuropsychopharmacology39(4): 944954.

Honer, W. G., P. Falkai, C. Chen, V. Arango, J. J. Mann and A. J. Dwork (1999). "Synaptic and plasticity-associated proteins in anterior frontal cortex in severe mental illness." Neuroscience91(4): 1247-1255.

Howe, C. L., S. Mayoral and M. Rodriguez (2006). "Activated microglia stimulate transcriptional changes in primary oligodendrocytes via IL-1beta." Neurobiol Dis 23(3): 731-739.

Howes, O., R. McCutcheon and J. Stone (2015). "Glutamate and dopamine in schizophrenia: an update for the 21st century." J Psychopharmacol29(2): 97-115. 
Hsu, R., A. Woodroffe, W. S. Lai, M. N. Cook, J. Mukai, J. P. Dunning, D. J. Swanson, J. L. Roos, G. R. Abecasis, M. Karayiorgou and J. A. Gogos (2007). "Nogo Receptor 1 (RTN4R) as a candidate gene for schizophrenia: analysis using human and mouse genetic approaches." PLoS One2(11): e1234.

Hu, X., C. W. Hicks, W. He, P. Wong, W. B. Macklin, B. D. Trapp and R. Yan (2006). "Bace1 modulates myelination in the central and peripheral nervous system." Nat Neurosci9(12): 1520-1525.

Hubl, D., T. Koenig, W. Strik, A. Federspiel, R. Kreis, C. Boesch, S. E. Maier, G. Schroth, K. Lovblad and T. Dierks (2004). "Pathways that make voices: white matter changes in auditory hallucinations." Arch Gen Psychiatry61(7): 658-668.

Insel, T. R. (2010). "Rethinking schizophrenia." Nature468(7321): 187-193.

Ishibashi, T., K. A. Dakin, B. Stevens, P. R. Lee, S. V. Kozlov, C. L. Stewart and R. D. Fields (2006). "Astrocytes promote myelination in response to electrical impulses." Neuron 49(6): 823-832.

Jahn, O., S. Tenzer and H. B. Werner (2009). "Myelin proteomics: molecular anatomy of an insulating sheath." Mol Neurobiol40(1): 55-72.

Kanfer, J., M. Parenty, C. Goujet-Zalc, M. Monge, L. Bernier, A. T. Campagnoni, A. Dautigny and B. Zalc (1989). "Developmental expression of myelin proteolipid, basic protein, and 2',3'-cyclic nucleotide 3'-phosphodiesterase transcripts in different rat brain regions." J Mol Neurosci1(1): 39-46.

Kao, H. T., R. M. Cawthon, L. E. Delisi, H. C. Bertisch, F. Ji, D. Gordon, P. Li, M. M. Benedict, W. M. Greenberg and B. Porton (2008). "Rapid telomere erosion in schizophrenia." Mol Psychiatry13(2): 118-119.

Kas, M. J., R. S. Kahn, D. A. Collier, J. L. Waddington, J. Ekelund, D. J. Porteous, K. Schughart and I. Hovatta (2011). "Translational neuroscience of schizophrenia: seeking a meeting of minds between mouse and man." Sci Transl Med3(102): 102mr103.

Katsel, P., K. L. Davis and V. Haroutunian (2005). "Variations in myelin and oligodendrocyte-related gene expression across multiple brain regions in schizophrenia: a gene ontology study." Schizophr Res79(2-3): 157-173.

Kaviani, H., J. A. Gray, S. A. Checkley, P. W. Raven, G. D. Wilson and V. Kumari (2004). "Affective modulation of the startle response in depression: influence of the severity of depression, anhedonia, and anxiety." J Affect Disord83(1): 21-31.

Kettenmann, H., U. K. Hanisch, M. Noda and A. Verkhratsky (2011). "Physiology of microglia." Physiol Rev91(2): 461-553.

Khandaker, G. M., L. Cousins, J. Deakin, B. R. Lennox, R. Yolken and P. B. Jones (2015). "Inflammation and immunity in schizophrenia: implications for pathophysiology and treatment." Lancet Psychiatry2(3): 258-270.

Kim, H., B. M. McGrath and P. H. Silverstone (2005). "A review of the possible relevance of inositol and the phosphatidylinositol second messenger system (PI-cycle) 
to psychiatric disorders--focus on magnetic resonance spectroscopy (MRS) studies." Hum Psychopharmacol20(5): 309-326.

Kirkpatrick, B., E. Messias, P. D. Harvey, E. Fernandez-Egea and C. R. Bowie (2008). "Is schizophrenia a syndrome of accelerated aging?" Schizophr Bull34(6): 1024-1032.

Klosterkötter, J., M. Hellmich, E. M. Steinmeyer and F. Schultze-Lutter (2001). "DIagnosing schizophrenia in the initial prodromal phase." Archives of General Psychiatry58(2): 158-164.

Klugmann, M., M. H. Schwab, A. Puhlhofer, A. Schneider, F. Zimmermann, I. R. Griffiths and K. A. Nave (1997). "Assembly of CNS myelin in the absence of proteolipid protein." Neuron 18(1): 59-70.

Knobler, R. L., J. G. Stempak and M. Laurencin (1976). "Nonuniformity of the oligodendroglial ensheathment of axons during myelination in the developing rat central nervous system. A serial section electron microscopical study." J Ultrastruct Res55(3): 417-432.

Koch, M. (1999). "The neurobiology of startle." Prog Neurobiol59(2): 107-128.

Kochunov, P., D. C. Glahn, L. M. Rowland, R. L. Olvera, A. Winkler, Y. H. Yang, H. Sampath, W. T. Carpenter, R. Duggirala, J. Curran, J. Blangero and L. E. Hong (2013). "Testing the hypothesis of accelerated cerebral white matter aging in schizophrenia and major depression." Biol Psychiatry73(5): 482-491.

Konrad, A. and G. Winterer (2008). "Disturbed structural connectivity in schizophrenia primary factor in pathology or epiphenomenon?" Schizophr Bull34(1): 72-92.

Kraguljac, N. V., M. Reid, D. White, R. Jones, J. den Hollander, D. Lowman and A. C. Lahti (2012). "Neurometabolites in schizophrenia and bipolar disorder - a systematic review and meta-analysis." Psychiatry Res203(2-3): 111-125.

Kramer-Albers, E. M., N. Bretz, S. Tenzer, C. Winterstein, W. Mobius, H. Berger, K. A. Nave, H. Schild and J. Trotter (2007). "Oligodendrocytes secrete exosomes containing major myelin and stress-protective proteins: Trophic support for axons?" Proteomics Clin Appl1(11): 1446-1461.

Kubicki, M., H. Park, C. F. Westin, P. G. Nestor, R. V. Mulkern, S. E. Maier, M. Niznikiewicz, E. E. Connor, J. J. Levitt, M. Frumin, R. Kikinis, F. A. Jolesz, R. W. McCarley and M. E. Shenton (2005). "DTI and MTR abnormalities in schizophrenia: analysis of white matter integrity." Neuroimage 26(4): 1109-1118.

Kuhn, P. L., E. Petroulakis, G. A. Zazanis and R. D. McKinnon (1995). "Motor function analysis of myelin mutant mice using a rotarod." Int J Dev Neurosci13(7): 715-722.

Kumari, V., M. Das, E. Zachariah, U. Ettinger and T. Sharma (2005). "Reduced prepulse inhibition in unaffected siblings of schizophrenia patients." Psychophysiology42(5): 588-594. 
Kumari, V., D. Fannon, M. A. Geyer, P. Premkumar, E. Antonova, A. Simmons and E. Kuipers (2008). "Cortical grey matter volume and sensorimotor gating in schizophrenia." Cortex 44(9): 1206-1214.

Lappe-Siefke, C., S. Goebbels, M. Gravel, E. Nicksch, J. Lee, P. E. Braun, I. R. Griffiths and K. A. Nave (2003). "Disruption of Cnp1 uncouples oligodendroglial functions in axonal support and myelination." Nat Genet33(3): 366-374.

Le Bihan, D., J. F. Mangin, C. Poupon, C. A. Clark, S. Pappata, N. Molko and H. Chabriat (2001). "Diffusion tensor imaging: concepts and applications." J Magn Reson Imaging13(4): 534-546.

Lebel, C., M. Gee, R. Camicioli, M. Wieler, W. Martin and C. Beaulieu (2012). "Diffusion tensor imaging of white matter tract evolution over the lifespan." Neuroimage60(1): 340-352.

Lee, S., M. K. Leach, S. A. Redmond, S. Y. Chong, S. H. Mellon, S. J. Tuck, Z. Q. Feng, J. M. Corey and J. R. Chan (2012). "A culture system to study oligodendrocyte myelination processes using engineered nanofibers." Nat Methods 9(9): 917-922.

Lee, Y., B. M. Morrison, Y. Li, S. Lengacher, M. H. Farah, P. N. Hoffman, Y. Liu, A. Tsingalia, L. Jin, P. W. Zhang, L. Pellerin, P. J. Magistretti and J. D. Rothstein (2012). "Oligodendroglia metabolically support axons and contribute to neurodegeneration." Nature487(7408): 443-448.

Li, J., E. Kale Edmiston, K. Chen, Y. Tang, X. Ouyang, Y. Jiang, G. Fan, L. Ren, J. Liu, Y. Zhou, W. Jiang, Z. Liu, K. Xu and F. Wang (2014). "A comparative diffusion tensor imaging study of corpus callosum subregion integrity in bipolar disorder and schizophrenia." Psychiatry Res221(1): 58-62.

Lisman, J. E., J. T. Coyle, R. W. Green, D. C. Javitt, F. M. Benes, S. Heckers and A. A. Grace (2008). "Circuit-based framework for understanding neurotransmitter and risk gene interactions in schizophrenia." Trends Neurosci31(5): 234-242.

Liu, J., K. Dietz, J. M. DeLoyht, X. Pedre, D. Kelkar, J. Kaur, V. Vialou, M. K. Lobo, D. M. Dietz, E. J. Nestler, J. Dupree and P. Casaccia (2012). "Impaired adult myelination in the prefrontal cortex of socially isolated mice." Nat Neurosci15(12): $1621-1623$.

Lowry, O. H., N. J. Rosebrough, A. L. Farr and R. J. Randall (1951). "Protein measurement with the Folin phenol reagent." J Biol Chem193(1): 265-275.

Makinodan, M., K. M. Rosen, S. Ito and G. Corfas (2012). "A critical period for social experience-dependent oligodendrocyte maturation and myelination." Science 337(6100): $1357-1360$.

Makinodan, M., T. Yamauchi, K. Tatsumi, H. Okuda, T. Takeda, K. Kiuchi, M. Sadamatsu, A. Wanaka and T. Kishimoto (2009). "Demyelination in the juvenile period, but not in adulthood, leads to long-lasting cognitive impairment and deficient social interaction in mice." Prog Neuropsychopharmacol Biol Psychiatry33(6): 978-985. 
Marsman, A., R. C. Mandl, D. W. Klomp, M. M. Bohlken, V. O. Boer, A. Andreychenko, W. Cahn, R. S. Kahn, P. R. Luijten and H. E. Hulshoff Pol (2014). "GABA and glutamate in schizophrenia: a 7 T (1)H-MRS study." Neuroimage Clin6: 398-407.

Martin, M., T. D. Hiltner, J. C. Wood, S. E. Fraser, R. E. Jacobs and C. Readhead (2006). "Myelin deficiencies visualized in vivo: visually evoked potentials and T2weighted magnetic resonance images of shiverer mutant and wild-type mice." $\underline{\mathrm{J}}$ Neurosci Res84(8): 1716-1726.

Martins-de-Souza, D., W. F. Gattaz, A. Schmitt, G. Maccarrone, E. Hunyadi-Gulyas, M. N. Eberlin, G. H. Souza, S. Marangoni, J. C. Novello, C. W. Turck and E. Dias-Neto (2009). "Proteomic analysis of dorsolateral prefrontal cortex indicates the involvement of cytoskeleton, oligodendrocyte, energy metabolism and new potential markers in schizophrenia." J Psychiatr Res43(11): 978-986.

Matthews, P. R., S. L. Eastwood and P. J. Harrison (2012). "Reduced myelin basic protein and actin-related gene expression in visual cortex in schizophrenia." PLoS One7(6): e38211.

Maxfield, F. R. and T. E. McGraw (2004). "Endocytic recycling." Nat Rev Mol Cell Biol5(2): 121-132.

McCullumsmith, R. E., D. Gupta, M. Beneyto, E. Kreger, V. Haroutunian, K. L. Davis and J. H. Meador-Woodruff (2007). "Expression of Transcripts for Myelination-Related Genes in the Anterior Cingulate Cortex in Schizophrenia." Schizophrenia research 90(13): $15-27$.

McGuire, P. K. and C. D. Frith (1996). "Disordered functional connectivity in schizophrenia." Psychol Med26(4): 663-667.

McIntosh, A. M., S. Munoz Maniega, G. K. Lymer, J. McKirdy, J. Hall, J. E. Sussmann, M. E. Bastin, J. D. Clayden, E. C. Johnstone and S. M. Lawrie (2008). "White matter tractography in bipolar disorder and schizophrenia." Biol Psychiatry64(12): 1088-1092.

McKenzie, I. A., D. Ohayon, H. Li, J. P. de Faria, B. Emery, K. Tohyama and W. D. Richardson (2014). "Motor skill learning requires active central myelination." Science 346(6207): 318-322.

Men, W., D. Falk, T. Sun, W. Chen, J. Li, D. Yin, L. Zang and M. Fan (2014). "The corpus callosum of Albert Einstein's brain: another clue to his high intelligence?" Brain 137(Pt 4): e268.

Mensch, S., M. Baraban, R. Almeida, T. Czopka, J. Ausborn, A. El Manira and D. A. Lyons (2015). "Synaptic vesicle release regulates myelin sheath number of individual oligodendrocytes in vivo." Nat Neurosci18(5): 628-630.

Miklyaeva, E. I., E. Castaneda and I. Q. Whishaw (1994). "Skilled reaching deficits in unilateral dopamine-depleted rats: impairments in movement and posture and compensatory adjustments." J Neurosci14(11 Pt 2): 7148-7158. 
Miller, D. J., T. Duka, C. D. Stimpson, S. J. Schapiro, W. B. Baze, M. J. McArthur, A. J. Fobbs, A. M. Sousa, N. Sestan, D. E. Wildman, L. Lipovich, C. W. Kuzawa, P. R. Hof and C. C. Sherwood (2012). "Prolonged myelination in human neocortical evolution." Proc Natl Acad Sci U S A109(41): 16480-16485.

Miron, V. E., A. Boyd, J. W. Zhao, T. J. Yuen, J. M. Ruckh, J. L. Shadrach, P. van Wijngaarden, A. J. Wagers, A. Williams, R. J. Franklin and C. ffrench-Constant (2013). "M2 microglia and macrophages drive oligodendrocyte differentiation during CNS remyelination." Nat Neurosci16(9): 1211-1218.

Morell, P. N., WT (1980). "Myelin." Scientific American242(5): 3.

Moscarello, M. (1997). "Myelin basic protein, the "executive" molecule of the myelin membrane." Cell Biology and Pathology of Myelin: Evolving Biological Concepts and therapeutic approaches: 13.

Mosebach, J., G. Keilhoff, T. Gos, K. Schiltz, L. Schoeneck, H. Dobrowolny, C. Mawrin, S. Muller, M. L. Schroeter, H. G. Bernstein, B. Bogerts and J. Steiner (2013). "Increased nuclear Olig1-expression in the pregenual anterior cingulate white matter of patients with major depression: a regenerative attempt to compensate oligodendrocyte loss?" J Psychiatr Res47(8): 1069-1079.

Moy, S. S., J. J. Nadler, A. Perez, R. P. Barbaro, J. M. Johns, T. R. Magnuson, J. Piven and J. N. Crawley (2004). "Sociability and preference for social novelty in five inbred strains: an approach to assess autistic-like behavior in mice." Genes Brain Behav3(5): 287-302.

Muller, C., N. M. Bauer, I. Schafer and R. White (2013). "Making myelin basic protein -from mRNA transport to localized translation." Front Cell Neurosci7: 169.

Myllykoski, M., L. Seidel, G. Muruganandam, A. Raasakka, A. E. Torda and P. Kursula (2015). "Structural and functional evolution of 2',3'-cyclic nucleotide 3'phosphodiesterase." Brain Res.

Nair, G., Y. Tanahashi, H. P. Low, S. Billings-Gagliardi, W. J. Schwartz and T. Q. Duong (2005). "Myelination and long diffusion times alter diffusion-tensor-imaging contrast in myelin-deficient shiverer mice." Neuroimage 28(1): 165-174.

Nave, K. A. and H. B. Werner (2014). "Myelination of the nervous system: mechanisms and functions." Annu Rev Cell Dev Biol30: 503-533.

Nawaz, S., A. Kippert, A. S. Saab, H. B. Werner, T. Lang, K. A. Nave and M. Simons (2009). "Phosphatidylinositol 4,5-bisphosphate-dependent interaction of myelin basic protein with the plasma membrane in oligodendroglial cells and its rapid perturbation by elevated calcium." J Neurosci29(15): 4794-4807.

Nayak, D., T. L. Roth and D. B. McGavern (2014). "Microglia development and function." Annu Rev Immunol32: 367-402.

Nestler, E. J. and S. E. Hyman (2010). "Animal models of neuropsychiatric disorders." Nat Neurosci13(10): 1161-1169. 
Nishiyama, A., M. Komitova, R. Suzuki and X. Zhu (2009). "Polydendrocytes (NG2 cells): multifunctional cells with lineage plasticity." Nat Rev Neurosci10(1): 9-22.

Orr, A. G., A. L. Orr, X. J. Li, R. E. Gross and S. F. Traynelis (2009). "Adenosine $\mathrm{A}(2 \mathrm{~A})$ receptor mediates microglial process retraction." Nat Neurosci12(7): 872-878.

Pajevic, S., P. J. Basser and R. D. Fields (2014). "Role of myelin plasticity in oscillations and synchrony of neuronal activity." Neuroscience 276: 135-147.

Parlapani, E., A. Schmitt, A. Erdmann, H. G. Bernstein, B. Breunig, O. Gruber, G. Petroianu, M. von Wilmsdorff, T. Schneider-Axmann, W. Honer and P. Falkai (2009). "Association between myelin basic protein expression and left entorhinal cortex prealpha cell layer disorganization in schizophrenia." Brain Res1301: 126-134.

Paus, T. (2005). "Mapping brain maturation and cognitive development during adolescence." Trends Cogn Sci9(2): 60-68.

Peferoen, L., M. Kipp, P. van der Valk, J. M. van Noort and S. Amor (2014). "Oligodendrocyte-microglia cross-talk in the central nervous system." Immunology141(3): 302-313.

Perlstein, W. M., C. S. Carter, D. C. Noll and J. D. Cohen (2001). "Relation of prefrontal cortex dysfunction to working memory and symptoms in schizophrenia." Am J Psychiatry158(7): 1105-1113.

Petersen, S. C. and K. R. Monk (2015). "Neurobiology: Myelin Goes Where the Action Is." Curr Biol25(13): R562-565.

Poliak, S. and E. Peles (2003). "The local differentiation of myelinated axons at nodes of Ranvier." Nat Rev Neurosci4(12): 968-980.

Port, J. D. and N. Agarwal (2011). "MR spectroscopy in schizophrenia." J Magn Reson Imaging34(6): 1251-1261.

Prabakaran, S., J. E. Swatton, M. M. Ryan, S. J. Huffaker, J. T. Huang, J. L. Griffin, M. Wayland, T. Freeman, F. Dudbridge, K. S. Lilley, N. A. Karp, S. Hester, D. Tkachev, M. L. Mimmack, R. H. Yolken, M. J. Webster, E. F. Torrey and S. Bahn (2004). "Mitochondrial dysfunction in schizophrenia: evidence for compromised brain metabolism and oxidative stress." Mol Psychiatry9(7): 684-697, 643.

Pribyl, T. M., C. W. Campagnoni, K. Kampf, T. Kashima, V. W. Handley, J. McMahon and A. T. Campagnoni (1993). "The human myelin basic protein gene is included within a 179-kilobase transcription unit: expression in the immune and central nervous systems." Proc Natl Acad Sci U S A90(22): 10695-10699.

Purger, D., E. M. Gibson and M. Monje (2015). "Myelin plasticity in the central nervous system." Neuropharmacology.

Qian, Y., G. Lei, F. X. Castellanos, H. Forssberg and R. D. Heijtz (2010). "Deficits in fine motor skills in a genetic animal model of ADHD." Behav Brain Funct6: 51. 
Quarles, R. M., WB; Morell, P (2006). "Myelin formation, structure and biochemistry." The biochemical Journal.

Readhead, C., B. Popko, N. Takahashi, H. D. Shine, R. A. Saavedra, R. L. Sidman and L. Hood (1987). "Expression of a myelin basic protein gene in transgenic shiverer mice: correction of the dysmyelinating phenotype." Cell48(4): 703-712.

Readhead, C., N. Takasashi, H. D. Shine, R. Saavedra, R. Sidman and L. Hood (1990). "Role of myelin basic protein in the formation of central nervous system myelin." Ann N Y Acad Sci605: 280-285.

Reif, A., A. Schmitt, S. Fritzen and K. P. Lesch (2007). "Neurogenesis and schizophrenia: dividing neurons in a divided mind?" Eur Arch Psychiatry Clin Neurosci257(5): 290-299.

Remahl, S. and C. Hildebrand (1982). "Changing relation between onset of myelination and axon diameter range in developing feline white matter." J Neurol Sci54(1): 33-45.

Ribeiro, A. S., B. A. Eales and F. G. Biddle (2013). "Short-term and long-term memory deficits in handedness learning in mice with absent corpus callosum and reduced hippocampal commissure." Behav Brain Res245: 145-151.

Richardson, W. D., N. Kessaris and N. Pringle (2006). "Oligodendrocyte wars." Nat Rev Neurosci7(1): 11-18.

Ross, C. A., R. L. Margolis, S. A. Reading, M. Pletnikov and J. T. Coyle (2006). "Neurobiology of schizophrenia." Neuron52(1): 139-153.

Roullet, P., A. Mele and M. Ammassari-Teule (1996). "Involvement of glutamatergic and dopaminergic systems in the reactivity of mice to spatial and non-spatial change." Psychopharmacology (Berl)126(1): 55-61.

Rowland, L. M., K. Kontson, J. West, R. A. Edden, H. Zhu, S. A. Wijtenburg, H. H. Holcomb and P. B. Barker (2013). "In vivo measurements of glutamate, GABA, and NAAG in schizophrenia." Schizophr Bull39(5): 1096-1104.

Roy, K., J. C. Murtie, B. F. El-Khodor, N. Edgar, S. P. Sardi, B. M. Hooks, M. BenoitMarand, C. Chen, H. Moore, P. O'Donnell, D. Brunner and G. Corfas (2007). "Loss of erbB signaling in oligodendrocytes alters myelin and dopaminergic function, a potential mechanism for neuropsychiatric disorders." Proc Natl Acad Sci U S A104(19): 81318136.

Saher, G., S. Quintes and K. A. Nave (2011). "Cholesterol: a novel regulatory role in myelin formation." Neuroscientist17(1): 79-93.

Sampaio-Baptista, C., A. A. Khrapitchev, S. Foxley, T. Schlagheck, J. Scholz, S. Jbabdi, G. C. DeLuca, K. L. Miller, A. Taylor, N. Thomas, J. Kleim, N. R. Sibson, D. Bannerman and H. Johansen-Berg (2013). "Motor skill learning induces changes in white matter microstructure and myelination." J Neurosci33(50): 19499-19503. 
Samuelsson, M., E. Skogh, K. Lundberg, M. Vrethem and K. Ollinger (2013). "Taurine and glutathione in plasma and cerebrospinal fluid in olanzapine treated patients with schizophrenia." Psychiatry Res210(3): 819-824.

Savonenko, A. V., T. Melnikova, F. M. Laird, K. A. Stewart, D. L. Price and P. C. Wong (2008). "Alteration of BACE1-dependent NRG1/ErbB4 signaling and schizophrenia-like phenotypes in BACE1-null mice." Proceedings of the National Academy of Sciences of the United States of America105(14): 5585-5590.

Sherman, D. L. and P. J. Brophy (2005). "Mechanisms of axon ensheathment and myelin growth." Nat Rev Neurosci6(9): 683-690.

Shine, H. D., C. Readhead, B. Popko, L. Hood and R. L. Sidman (1992). "Morphometric analysis of normal, mutant, and transgenic CNS: correlation of myelin basic protein expression to myelinogenesis." J Neurochem58(1): 342-349.

Shivaraj, M. C., G. Marcy, G. Low, J. R. Ryu, X. Zhao, F. J. Rosales and E. L. Goh (2012). "Taurine induces proliferation of neural stem cells and synapse development in the developing mouse brain." PLoS One7(8): e42935.

Simons, M. and K. A. Nave (2015). "Oligodendrocytes: Myelination and Axonal Support." Cold Spring Harb Perspect Biol.

Simons, M. and J. Trotter (2007). "Wrapping it up: the cell biology of myelination." Curr Opin Neurobiol17(5): 533-540.

Snaidero, N., W. Mobius, T. Czopka, L. H. Hekking, C. Mathisen, D. Verkleij, S. Goebbels, J. Edgar, D. Merkler, D. A. Lyons, K. A. Nave and M. Simons (2014). "Myelin membrane wrapping of CNS axons by PI(3,4,5)P3-dependent polarized growth at the inner tongue." Cell156(1-2): 277-290.

Sobottka, B., U. Ziegler, A. Kaech, B. Becher and N. Goebels (2011). "CNS live imaging reveals a new mechanism of myelination: the liquid croissant model." Glia59(12): 1841-1849.

Sofroniew, M. V. and H. V. Vinters (2010). "Astrocytes: biology and pathology." Acta Neuropathol119(1): 7-35.

Song, S. K., S. W. Sun, M. J. Ramsbottom, C. Chang, J. Russell and A. H. Cross (2002). "Dysmyelination revealed through MRI as increased radial (but unchanged axial) diffusion of water." Neuroimage17(3): 1429-1436.

Spies, M., G. M. Knudsen, R. Lanzenberger and S. Kasper (2015). "The serotonin transporter in psychiatric disorders: insights from PET imaging." Lancet Psychiatry2 (8): 743-755.

Steen, R. G., R. M. Hamer and J. A. Lieberman (2005). "Measurement of brain metabolites by $1 \mathrm{H}$ magnetic resonance spectroscopy in patients with schizophrenia: a systematic review and meta-analysis." Neuropsychopharmacology30(11): 1949-1962.

Stefansson, H., E. Sigurdsson, V. Steinthorsdottir, S. Bjornsdottir, T. Sigmundsson, S. Ghosh, J. Brynjolfsson, S. Gunnarsdottir, O. Ivarsson, T. T. Chou, O. Hjaltason, B. 
Birgisdottir, H. Jonsson, V. G. Gudnadottir, E. Gudmundsdottir, A. Bjornsson, B. Ingvarsson, A. Ingason, S. Sigfusson, H. Hardardottir, R. P. Harvey, D. Lai, M. Zhou, D. Brunner, V. Mutel, A. Gonzalo, G. Lemke, J. Sainz, G. Johannesson, T. Andresson, D. Gudbjartsson, A. Manolescu, M. L. Frigge, M. E. Gurney, A. Kong, J. R. Gulcher, H. Petursson and K. Stefansson (2002). "Neuregulin 1 and susceptibility to schizophrenia." Am J Hum Genet71(4): 877-892.

Stevens, B., S. Porta, L. L. Haak, V. Gallo and R. D. Fields (2002). "Adenosine: a neuron-glial transmitter promoting myelination in the CNS in response to action potentials." Neuron36(5): 855-868.

Stevens, B., S. Tanner and R. D. Fields (1998). "Control of myelination by specific patterns of neural impulses." J Neurosci18(22): 9303-9311.

Stikov, N., J. S. W. Campbell, T. Stroh, M. Lavelée, S. Frey, J. Novek, S. Nuara, M.-K. Ho, B. J. Bedell, R. F. Dougherty, I. R. Leppert, M. Boudreau, S. Narayanan, T. Duval, J. Cohen-Adad, P.-A. Picard, A. Gasecka, D. Côté and G. B. Pike (2015). "In vivo histology of the myelin g-ratio with magnetic resonance imaging." NeuroImage118: 397-405.

Sturrock, R. R. (1980). "Myelination of the mouse corpus callosum." Neuropathology and applied neurobiology6: 6 .

Sturrock, R. R. (1987). "Ultrastructural evidence for the mitotic division of neurons in the anterior horn of the fetal rabbit in the later stages of gestation." J Anat 155: 63-75.

Sullivan, P. F., M. J. Daly and M. O'Donovan (2012). "Genetic architectures of psychiatric disorders: the emerging picture and its implications." Nat Rev Genet13(8): 537-551.

Sun, J., J. J. Maller, L. Guo and P. B. Fitzgerald (2009). "Superior temporal gyrus volume change in schizophrenia: a review on region of interest volumetric studies." Brain Res Rev61(1): 14-32.

Sussmann, J. E., G. K. Lymer, J. McKirdy, T. W. Moorhead, S. Munoz Maniega, D. Job, J. Hall, M. E. Bastin, E. C. Johnstone, S. M. Lawrie and A. M. McIntosh (2009). "White matter abnormalities in bipolar disorder and schizophrenia detected using diffusion tensor magnetic resonance imaging." Bipolar Disord11(1): 11-18.

Symms, M., H. R. Jager, K. Schmierer and T. A. Yousry (2004). "A review of structural magnetic resonance neuroimaging." J Neurol Neurosurg Psychiatry75(9): 1235-1244.

Takahashi, N., T. Sakurai, K. L. Davis and J. D. Buxbaum (2011). "Linking oligodendrocyte and myelin dysfunction to neurocircuitry abnormalities in schizophrenia." Prog Neurobiol93(1): 13-24.

Takanashi, J., N. Nitta, N. Iwasaki, S. Saito, R. Tanaka, A. J. Barkovich and I. Aoki (2014). "Neurochemistry in shiverer mouse depicted on MR spectroscopy." J Magn Reson Imaging39(6): 1550-1557.

Takeuchi, N., Y. Oouchida and S. Izumi (2012). "Motor control and neural plasticity through interhemispheric interactions." Neural Plast2012: 823285. 
Tanaka, H., J. Ma, K. F. Tanaka, K. Takao, M. Komada, K. Tanda, A. Suzuki, T. Ishibashi, H. Baba, T. Isa, R. Shigemoto, K. Ono, T. Miyakawa and K. Ikenaka (2009). "Mice with Altered Myelin Proteolipid Protein Gene Expression Display Cognitive Deficits Accompanied by Abnormal Neuron-Glia Interactions and Decreased Conduction Velocities." The Journal of Neuroscience29(26): 8363-8371.

Tanaka, R., N. Iwasaki, M. Hayashi, J. Nakayama, T. Ohto, M. Takahashi, T. Numano, K. Homma, K. Hamano and R. Sumazaki (2012). "Abnormal brain MRI signal in 18qsyndrome not due to dysmyelination." Brain Dev34(3): 234-237.

Taveggia, C., P. Thaker, A. Petrylak, G. L. Caporaso, A. Toews, D. L. Falls, S. Einheber and J. L. Salzer (2008). "Type III neuregulin-1 promotes oligodendrocyte myelination." Glia56(3): 284-293.

Taylor, M. J., S. Selvaraj, R. Norbury, P. Jezzard and P. J. Cowen (2009). "Normal glutamate but elevated myo-inositol in anterior cingulate cortex in recovered depressed patients." J Affect Disord119(1-3): 186-189.

Tkachev, D., M. L. Mimmack, M. M. Ryan, M. Wayland, T. Freeman, P. B. Jones, M. Starkey, M. J. Webster, R. H. Yolken and S. Bahn (2003). "Oligodendrocyte dysfunction in schizophrenia and bipolar disorder." Lancet362(9386): 798-805.

Tononi, G. and C. Cirelli (2014). "Sleep and the price of plasticity: from synaptic and cellular homeostasis to memory consolidation and integration." Neuron81(1): 12-34.

Trajkovic, K., A. S. Dhaunchak, J. T. Goncalves, D. Wenzel, A. Schneider, G. Bunt, K. A. Nave and M. Simons (2006). "Neuron to glia signaling triggers myelin membrane exocytosis from endosomal storage sites." J Cell Biol172(6): 937-948.

Tu, T. W., J. H. Kim, F. Q. Yin, L. B. Jakeman and S. K. Song (2013). "The impact of myelination on axon sparing and locomotor function recovery in spinal cord injury assessed using diffusion tensor imaging." NMR Biomed26(11): 1484-1495.

Tyszka, J. M., C. Readhead, E. L. Bearer, R. G. Pautler and R. E. Jacobs (2006). "Statistical diffusion tensor histology reveals regional dysmyelination effects in the shiverer mouse mutant." Neuroimage29(4): 1058-1065.

Uhlhaas, P. J. and W. Singer (2010). "Abnormal neural oscillations and synchrony in schizophrenia." Nat Rev Neurosci11(2): 100-113.

Umemori, H., S. Sato, T. Yagi, S. Aizawa and T. Yamamoto (1994). "Initial events of myelination involve Fyn tyrosine kinase signalling." Nature367(6463): 572-576.

Uranova, N., D. Orlovskaya, O. Vikhreva, I. Zimina, N. Kolomeets, V. Vostrikov and V. Rachmanova (2001). "Electron microscopy of oligodendroglia in severe mental illness." Brain Res Bull55(5): 597-610.

Uranova, N. A., V. M. Vostrikov, D. D. Orlovskaya and V. I. Rachmanova (2004). "Oligodendroglial density in the prefrontal cortex in schizophrenia and mood disorders: a study from the Stanley Neuropathology Consortium." Schizophr Res67(2-3): 269-275. 
Uranova, N. A., V. M. Vostrikov, O. V. Vikhreva, I. S. Zimina, N. S. Kolomeets and D. D. Orlovskaya (2007). "The role of oligodendrocyte pathology in schizophrenia." Int J Neuropsychopharmacol10(4): 537-545.

van Berckel, B. N., M. G. Bossong, R. Boellaard, R. Kloet, A. Schuitemaker, E. Caspers, G. Luurtsema, A. D. Windhorst, W. Cahn, A. A. Lammertsma and R. S. Kahn (2008). "Microglia activation in recent-onset schizophrenia: a quantitative (R)[11C]PK11195 positron emission tomography study." Biol Psychiatry64(9): 820-822.

van Os, J. and S. Kapur (2009). "Schizophrenia." Lancet374(9690): 635-645.

van Os, J., G. Kenis and B. P. Rutten (2010). "The environment and schizophrenia." Nature468(7321): 203-212.

Vostrikov, V. and N. Uranova (2011). "Age-related increase in the number of oligodendrocytes is dysregulated in schizophrenia and mood disorders." $\underline{\text { Schizophr Res }}$ Treatment2011: 174689.

Wahl, M., B. Lauterbach-Soon, E. Hattingen, P. Jung, O. Singer, S. Volz, J. C. Klein, H. Steinmetz and U. Ziemann (2007). "Human motor corpus callosum: topography, somatotopy, and link between microstructure and function." J Neurosci27(45): 1213212138.

Wake, H., P. R. Lee and R. D. Fields (2011). "Control of local protein synthesis and initial events in myelination by action potentials." Science 333(6049): 1647-1651.

Walker, E., L. Kestler, A. Bollini and K. M. Hochman (2004). "Schizophrenia: etiology and course." Annu Rev Psychol55: 401-430.

Walther, S. and W. Strik (2012). "Motor symptoms and schizophrenia." Neuropsychobiology66(2): 77-92.

Waxman, S. G. and H. A. Swadlow (1976). "Ultrastructure of visual callosal axons in the rabbit." Exp Neurol53(1): 115-127.

Weiss, M. D., J. Hammer and R. H. Quarles (2000). "Oligodendrocytes in aging mice lacking myelin-associated glycoprotein are dystrophic but not apoptotic." J Neurosci Res62(6): 772-780.

Welham, J., M. Isohanni, P. Jones and J. McGrath (2009). "The antecedents of schizophrenia: a review of birth cohort studies." Schizophr Bull35(3): 603-623.

Werner, H. B. and O. Jahn (2010). "Myelin matters: proteomic insights into white matter disorders." Expert Rev Proteomics7(2): 159-164.

Wesseling, H., M. G. Gottschalk and S. Bahn (2015). "Targeted multiplexed selected reaction monitoring analysis evaluates protein expression changes of molecular risk factors for major psychiatric disorders." Int J Neuropsychopharmacol18(1).

White, R., C. Gonsior, E. M. Kramer-Albers, N. Stohr, S. Huttelmaier and J. Trotter (2008). "Activation of oligodendroglial Fyn kinase enhances translation of mRNAs transported in hnRNP A2-dependent RNA granules." J Cell Biol181(4): 579-586. 
White, R. and E. M. Kramer-Albers (2014). "Axon-glia interaction and membrane traffic in myelin formation." Front Cell Neurosci7: 284.

Whitford, T. J., J. M. Ford, D. H. Mathalon, M. Kubicki and M. E. Shenton (2012). "Schizophrenia, myelination, and delayed corollary discharges: a hypothesis." Schizophr Bull38(3): 486-494.

Whitford, T. J., D. H. Mathalon, M. E. Shenton, B. J. Roach, R. Bammer, R. A. Adcock, S. Bouix, M. Kubicki, J. De Siebenthal, A. C. Rausch, J. S. Schneiderman and J. M. Ford (2011). "Electrophysiological and diffusion tensor imaging evidence of delayed corollary discharges in patients with schizophrenia." Psychol Med41(5): 959969.

Winterstein, C., J. Trotter and E. M. Kramer-Albers (2008). "Distinct endocytic recycling of myelin proteins promotes oligodendroglial membrane remodeling." J Cell Sci121(Pt 6): 834-842.

$\mathrm{Wu}$, J. Y. and H. Prentice (2010). "Role of taurine in the central nervous system." $\underline{\mathbf{J}}$ Biomed Sci17 Suppl 1: S1.

Xin, M., T. Yue, Z. Ma, F. F. Wu, A. Gow and Q. R. Lu (2005). "Myelinogenesis and axonal recognition by oligodendrocytes in brain are uncoupled in Olig1-null mice." $\underline{\mathrm{J}}$ Neurosci25(6): 1354-1365.

Xiong, P., Y. Zeng, Q. Wu, D. X. Han Huang, H. Zainal, X. Xu, J. Wan, F. Xu and J. $\mathrm{Lu}$ (2014). "Combining serum protein concentrations to diagnose schizophrenia: a preliminary exploration." J Clin Psychiatry75(8): e794-801.

Yang, L., E. M. Kan, J. Lu, C. Wu and E. A. Ling (2014). "Expression of 2',3'-cyclic nucleotide 3'-phosphodiesterase (CNPase) and its roles in activated microglia in vivo and in vitro." J Neuroinflammation11: 148.

Yang, S., C. Li, X. Qiu, L. Zhang, W. Lu, L. Chen, Y. Y. Zhao, X. Y. Shi, C. X. Huang, G. H. Cheng and Y. Tang (2013). "Effects of an enriched environment on myelin sheaths in the white matter of rats during normal aging: A stereological study." Neuroscience 234: 13-21.

Young, K. M., K. Psachoulia, R. B. Tripathi, S. J. Dunn, L. Cossell, D. Attwell, K. Tohyama and W. D. Richardson (2013). "Oligodendrocyte dynamics in the healthy adult CNS: evidence for myelin remodeling." Neuron77(5): 873-885.

Zalesky, A., A. Fornito, M. L. Seal, L. Cocchi, C. F. Westin, E. T. Bullmore, G. F. Egan and C. Pantelis (2011). "Disrupted axonal fiber connectivity in schizophrenia." Biol Psychiatry69(1): 80-89.

Zammit, S., P. Allebeck, S. Andreasson, I. Lundberg and G. Lewis (2002). "Self reported cannabis use as a risk factor for schizophrenia in Swedish conscripts of 1969: historical cohort study." BMJ : British Medical Journal325(7374): 1199-1199.

Zuchero, J. B. and B. A. Barres (2011). "Between the sheets: a molecular sieve makes myelin membranes." Dev Cell21(3): 385-386. 
Zuchero, J. B., M. M. Fu, S. A. Sloan, A. Ibrahim, A. Olson, A. Zaremba, J. C. Dugas, S. Wienbar, A. V. Caprariello, C. Kantor, D. Leonoudakus, K. Lariosa-Willingham, G. Kronenberg, K. Gertz, S. H. Soderling, R. H. Miller and B. A. Barres (2015). "CNS myelin wrapping is driven by actin disassembly." Dev Cell34(2): 152-167.

Zwingmann, C., D. Leibfritz and A. S. Hazell (2003). "Energy metabolism in astrocytes and neurons treated with manganese: relation among cell-specific energy failure, glucose metabolism, and intercellular trafficking using multinuclear NMRspectroscopic analysis." J Cereb Blood Flow Metab23(6): 756-771. 




\section{CURRICULUM VITAE}

Giulia Poggi

15.02.1986, Novara - Italy

Current address: Innersteweg, 15

37081 Göttingen - Germany

Mobile number (DE): 004915146411235

E-mail address: giulia.poggi86@gmail.com

Mother tongue: Italian

Other languages: English (fluent)

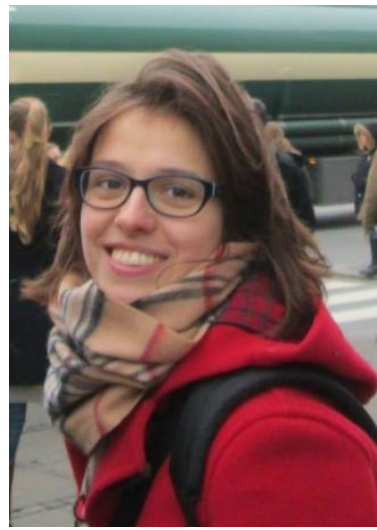

\section{Research Interest}

Clinical and Molecular Neuroscience, especially in the context of severe psychiatric diseases.

\section{Education}

Since 11/2011: PhD student

Clinical Neuroscience

Max Planck Institute of Experimental Medicine

Supervisor: Prof. Hannelore Ehrenreich

Thesis Committee Members: Prof. Klaus-Armin Nave, Prof. Mikael Simons

Main research topic: "Sub-phenotype in psychiatric diseases"

2010/2011: $\quad$ MSc Molecular Neuroscience

University of Bristol - Laboratories for Integrative Neuroscience and Endocrinology

Supervisor: Dr. Liang-Fong Wong

Thesis Title: "Developing a novel regulatable system in lentiviral vectors"

Degree with MERIT

2008/2010: $\quad$ MSc Pharmaceutical Biotechnology

Università del Piemonte Orientale "A.Avogadro"

Supervisor: Prof. Marco Orsetti

Thesis title: "Preclinical study on the role of the serotoninergic 5HT1A system in major depressive disorder" Grade: 110/110

2005/2008: $\quad$ BA Biotechnology

Università del Piemonte Orientale "A.Avogadro"

Supervisor: Prof. Marco Orsetti

"Gene expression profile during exploration of the environment"

Grade: $102 / 110$

\section{$\underline{\text { Scientific skills and experiences }}$}

- Mouse Behaviour (basic behaviour, high brain function and fine motor tasks)

- Mouse perfusion for immunohistochemistry

- Immunohistochemistry

- Fresh brain tissue preparation

- DNA, RNA, protein extraction

- PCR, qPCR, retrotranscription 
- Western blotting

- Cloning

- Cell culture

- Transfection

- Luciferase Assay

- Transduction (3 months-experience at the University of Bristol)

- Mouse primary microglia preparation

- January/February 2014 - Conventional electron microscopy in collaboration and under the guidance of Dr. Möbius and colleagues - EM facility, MPIEM Göttingen

- August 2013 - Mouse brain imaging in collaboration and under the guidance of Prof. Susann Boretius, "Department of Radiology and Neuroradiology" - University of Kiel.

- (Experience on Amira software)

- March-May 2009 - In vitro ubiquitination and pull-down. Lab rotation experience under the guidance of Dr. Lorenza Penengo - Università del Piemonte Orientale, Novara

\section{Teaching skills}

June 2015: Translational Neuroscience: Schizophrenia - workshop

Neuroimaging - focus on morphometrical analysis

Neurophysiology (EEG, ERP)

Practical part: Neuroimaging

Nov 2014-May 2015: Co-supervision of a Master Student

Project: "Brain inflammation as a common denominator in mouse models of neuropsychiatric disease based on deficiencies of myelination or autophagy"

June 2014: Translational Neuroscience: Schizophrenia - workshop

Neuroimaging - focus on morphometrical analysis

Neurophysiology (EEG, ERP)

Practical part: Neuroimaging

May-June 2014: Supervision of a Lab Rotation Student

Project: "Behavioural characterisation of Myelin Basic Protein (MBP) hypo-morph mouse"

November 2013: Translational Neuroscience: Multiple Sclerosis - workshop

Practical part: Mouse Behaviour Neurophysiology (EEG, ERP)

Practical part: Neuroimaging

June 2013: Translational Neuroscience: Schizophrenia - workshop

Neurophysiology (EEG, ERP)

Practical part: PCR, cell culture

November 2012: Translational Neuroscience: Multiple Sclerosis - workshop

Neurophysiology (EEG, ERP)

Practical part: Mouse Behaviour

\section{Other skills}

Sep - Nov 2014: Writing in the sciences - Online Stanford Course - certificate with DISTINCTION

2014 - 2015: PhD representative of the MPI of Experimental Medicine, Göttingen

2014 - 2015: Member of the PhDnet survey group - Max Planck Society

2010 - 2011: Member of the "Green Group" - University of Bristol, Dorothy Hodgkin Building

May 2011: Cancer Research UK - fundraising via "Race for Life" 10K running race 


\section{Posters and publications}

\section{Conference posters:}

May 2015 - Neurizon 2015 - Poster Presentation:

Myelin abnormalities in mental disease, cause or consequence

Poggi G, Boretius S, Möbius W, Hassouna I, Moschny N, Nave KA, Ehrenreich H

March 2015 - 11 th Göttingen Meeting of the German Neuroscience Society - Poster Presentation:

Myelin abnormalities in mental disease, cause or consequence

Poggi G, Boretius S, Möbius W, Hassouna I, Moschny N, Nave KA, Ehrenreich H

September 2014 - GBM study group molecular Neurobiology - Perspective of molecular neuroscience in health and disease" - Poster presentation:

Myelin abnormalities in mental disease, focus on myelin basic protein (MBP)

Poggi G, Boretius S, Möbius W, Nave KA, Ehrenreich H.

\section{Publications:}

Cortical network dysfunction caused by a subtle defect of myelination

Poggi G*, Boretius S, Möbius W, Moschny N, Hassouna I, Ruhwedel T, Baudewig J, Nave KA, Ehrenreich H. Manuscript in preparation ( ${ }^{*}$ first authorship).

Accumulated common variants in the broader fragile $X$ gene family modulate autistic phenotypes

Stepniak $B^{*}$, Kästner $A^{*}$, Poggi $G^{*}$, Mitjans M, Begemann M, Hartmann A, Van der Auwera S, Sananbenesi F, KruegerBurg D, Matuszko G, Brosi C, Homuth G, Völzke H, Benseler F, Bagni C, Fischer U, Dityatev A, Grabe HJ, Rujescu D, Fischer A, Ehrenreich $\mathrm{H}$.

EMBO Molecular Medicine, in press (*shared first authorship)

The brain as immunoprecipitator of serum autoantibodies directed against the NMDAR subunit NR1

Castillo-Gomez E, Kästner A, Steiner J, Weber F, Hettling B, Poggi G, Asif AR, Pfander V, Gámez Viana MA, Hammer C, Schulz T, Binder L, Stöcker W, Schneider A, Ehrenreich H.

Annals of Neurology, 2015 Oct 27.

Fast Cerebellar Reflex Circuitry Requires Synaptic Vesicle Priming by Munc13-3.

Netrakanti PR, Cooper BH, Dere E,Poggi G, Winkler D, Brose N, Ehrenreich H.

Cerebellum. 2015 Jan 24

Gpm6b deficiency impairs sensorimotor gating and modulates the behavioral response to a $5-H T 2 A / C$ receptor agonist. Dere E, Winkler D, Ritter C, Ronnenberg A,Poggi G, Patzig J, Gernert M, Müller C, Nave KA, Ehrenreich H, Werner HB. Behav Brain Res. 2015 Jan 15; 277:254-63.

\section{Personal Interest}

Volleyball $\quad 2006$ - 2007 Gattinara volley - FIPAV;

2006 - 2007 Università del Piemonte Orientale - Pharmacy faculty team;

2007 - 2010 San Giacomo Club, Novara - FIPAV;

2010 - 2011 University of Bristol volleyball team;

2012 - 2015 ASC Göttingen - landesliga;

2015 (ongoing) ASC Göttingen - verbandsliga

Classic literature, Walt Disney Animations 
